\title{
High Quantum Efficiency Ultraviolet Avalanche Photodiode
}

\author{
Dissertation \\ Presented to \\ the faculty of the School of Engineering and Applied Science \\ University of Virginia \\ In Partial Fulfillment \\ of the requirements for the Degree \\ Doctor of Philosophy \\ Electrical Engineering \\ by
}

Yaojia Chen

May 2016 
C Copyright by

Yaojia Chen

All rights reserved

May 2016 


\section{APPROVAL SHEET}

The dissertation

is submitted in partial fulfillment of the requirements

for the degree of

Doctor of Philosophy in Electrical Engineering

Yaojia Chen

The dissertation has been read and approved by the examining Committee:

Dr. Joe C. Campbell (Advisor)

$\overline{\text { Dr. Archie L. Holmes Jr. (Committee chair) }}$

$\overline{\text { Dr. Anand Sampath }}$

$\overline{\text { Dr. Andreas Beling }}$

Dr. Art Lichtenberger

Accepted for the School of Engineering and Applied Science:

Dr. Craig H. Benson, Dean, School of Engineering and Applied Science

May 2016 
This thesis is dedicated

to my parents 


\section{Abstract}

Ultraviolet (UV) radiation covers the wavelength range from $10 \mathrm{~nm}$ to $400 \mathrm{~nm}$. The detection of UV radiation presents a wide range of civil and military applications, such as chemical and biological analysis, flame detection, inter-satellite communications and astronomical studies. For many decades, the detection of UV light has been accomplished primarily with photomultiplier tubes (PMT), which have the beneficial characteristics of high sensitivity with low noise. But they are also fragile, need large power sources, which make systems bulky and heavy, and are relatively expensive. For some application, avalanche photodiodes, which are semiconductor detectors, are potential replacements for PMTs. Among all the material candidates, $4 \mathrm{H}-\mathrm{SiC}$ has the most promising material properties and APDs based on $\mathrm{SiC}$ have achieved excellent performance with high sensitivity and good robustness. However their relatively narrow spectral response has limited their use for deep-UV and near-UV detection. This dissertation describes my work on the deep-UV enhancement of SiC-based APDs and a study of GaAs/AlGaAs-based APDs to achieve comparable dark current, multiplication gain and excess noise performance to $\mathrm{SiC}$ but with enhanced responsivity for near-UV detection.

From my study of the spectral response of SiC PIN-structure APDs, the weak deep-UV response was found to be related to the high surface recombination velocity at the top surface and the short diffusion length in the top highly doped layer. Two different approaches were investigated to enhance the deep-UV response of these SiC APDs. First, 
a SiC metal-n`-p structure was examined. By replacing the highly doped top layer with a semitransparent metal contact in the active area, deep-UV photons are absorbed within the high electric field depletion region. Photodiodes with external quantum efficiency (EQE) greater than $\sim 40 \%$ at wavelength between $200 \mathrm{~nm}$ and $235 \mathrm{~nm}$ were demonstrated with this structure. Second, an NIP structure with a graded doped top layer was studied. The highlow junction formed due to the graded doping in the top layer is expected to provide an electric field that pushes carriers away from the surface and, thus, suppresses surface recombination. However no significant improvement in the deep-UV response was observed. The possible reason is that the electric field generated by the high-low junction is not large enough to compensate the electric field in the opposite direction induced by the surface band bending.

As an alternative method to achieve high deep-UV response, while keeping $\mathrm{SiC}$ as multiplication layer material, an $\mathrm{AlGaN} / \mathrm{SiC}$ heterogeneous structure photodiode was explored. As early effort, a separate absorption and multiplication (SAM) structure was utilized so that the spectral response could be tuned by changing the $\mathrm{Al}$ mode fraction in the AlGaN absorption layer. However, it was found that the electric field is confined in the $\mathrm{SiC}$ multiplication region by the polarization-induced charge at the $\mathrm{AlGaN} / \mathrm{SiC}$ interface. Since there is a lattice mismatch between $\mathrm{AlGaN}$ and $\mathrm{SiC}$, high defect densities were found in the AlGaN layers. Consequently, an electric field is needed in this layer to achieve good collection efficiency. An AIN inter-layer was simulated to be effective in introducing an electric field in the AlGaN layer, but experimental results showed that the AlN acted as a barrier that prevented carriers in the $\mathrm{AlGaN}$ absorption layer from entering the $\mathrm{SiC}$ multiplication layer. To overcome this problem, an AlGaN top layer with higher Al fraction 
was used. With a bandgap of over $5.38 \mathrm{eV}$, this layer is transparent to the deep-UV photons. The photodiodes with this structure achieved high peak external quantum efficiency of $\sim 76 \%$ at $242 \mathrm{~nm}$.

The second part of my work focused on near-UV detection using the GaAs/AlGaAs material system. $\mathrm{Al}_{0.8} \mathrm{Ga}_{0.2} \mathrm{As}$, with a band gap of $2.23 \mathrm{eV}$, is a promising candidate to replace $\mathrm{Si}$ for near-UV detection. An $\mathrm{Al}_{0.8} \mathrm{Ga}_{0.2} \mathrm{As}$ PIN-structure APD was designed, fabricated, and characterized. These APDs have exhibited very low dark current of 179 $\mathrm{nA} / \mathrm{cm}^{2}$ at gain as high as 200 . Also the measured excess noise factor was very low. The $\mathrm{k}$ factor, which is the ratio of the electron ionization coefficient, $\alpha$, to that of the holes, $\beta$, is about 0.15 , which is comparable to $\mathrm{Si}$. The peak external quantum efficiency was $\sim 26 \%$ at $470 \mathrm{~nm}$. The response in the near-UV was lower than the expectation. Secondary ion mass spectrometry (SIMS) measurements showed that the doping in the p- layer is significantly higher than the specification that was sent to the foundry. The relatively low near-UV response is due to the fact that a large fraction of the carriers were generated in the undepleted p- layer.

My final research project was to utilize recessed window and surface texturing techniques to enhance the near-UV response of GaAs/AlGaAs photodiodes. The surface texturing using nanosphere natural lithography provided a photodiode surface with very low reflectivity $(<5 \%)$ across a wide spectrum. Combined with a recessed window structure, the GaAs/AlGaAs photodiode exhibited an external quantum efficiency in the range $45 \%$ to $55 \%$ from $300 \mathrm{~nm}$ to $850 \mathrm{~nm}$. These photodiodes were fabricated into arrays and sent to NASA for a project to reduce the number of cameras in satellites. 


\section{Acknowledgement}

First of all, I would like to express my great gratitude to my $\mathrm{PhD}$ advisor Dr. Joe C. Campbell for his outstanding mentorship and support over the past several years. I benefit a lot from his vast technical expertise and insight, as well as his high standards in research.

I would like to thank the rest of my advising committee Dr. Archie Holmes, Dr. Andreas Beling, Dr. Arthur Lichtenberger and Dr. Anand Sampath, for their valuable advice and critical reading of this dissertation.

I would also like to thank my fellow group members including Dr. Qiugui Zhou, Dr. Dion McIntosh-Dorsey, Dr. Xiaoguang Zheng, Dr. Wenlu Sun, Dr. Min Ren, Dr. Zhiwen Lu, Dr. Lijun Li, Dr. Kejia Li, Dr. Yang Fu, Dr. Yang Shen, Dr. Xiaojun Xie, Qinglong Li, Zhanyu Yang, Maddison Woodson, Ye Wang, Jizhao Zang, Qianhuan Yu and Junming Mao for being great friends and research collaborators. In particular, I would like to thank Dr. Qiugui Zhou and Dr. Dion McIntosh-Dorsey for their mentorship at the beginning of my research, Dr. Wenlu Sun and Dr. Min Ren for the cooperation on the AlGaAs projects, and Dr. Xiaoguang Zheng for continuous help and advice for my research.

I would like to give my special thanks to Dr. Anand Sampath, Dr. Michael Wraback, Dr. Chad Gallinat and Dr. Lee Rodack of the U.S Army Research Laboratory and Dr. Xiaojie Chen of Radiation Monitoring Devices for their great support and collaboration,

which has been essentially important to my work on the $\mathrm{AlGaN} / \mathrm{SiC}$ projects and $\mathrm{AlGaAs}$ projects. 
Last, but far from the least, I would like to thank all the staff members of the Microfabrication Lab at University of Virginia who have enabled all the devices fabricated in this dissertation by their hard work. 


\section{Table of Contents}

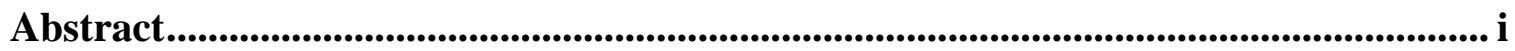

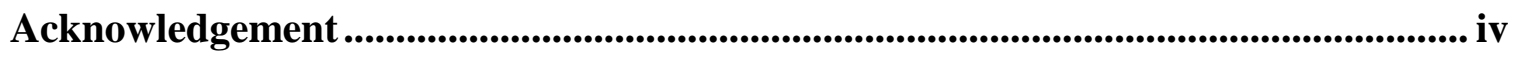

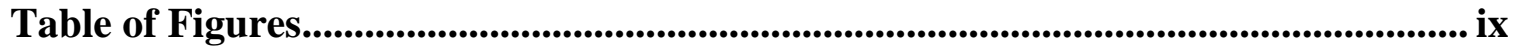

Chapter 1. Introduction .................................................................................... 1

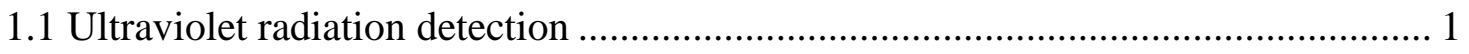

1.2 Material candidates for UV detection ............................................................. 2

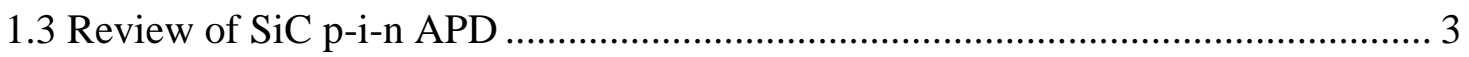

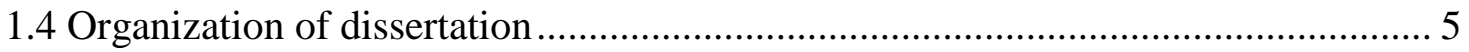

Chapter 2. Fabrication and characterization of photodetectors .................................. 6

2.1 Fabrication of III-Nitride/SiC photodiodes...................................................... 6

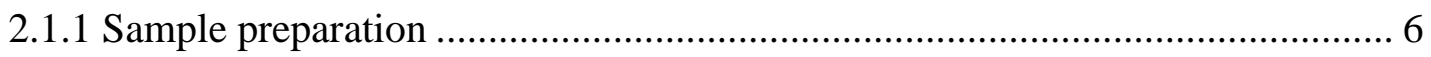

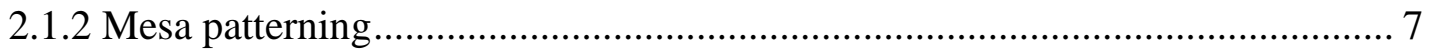

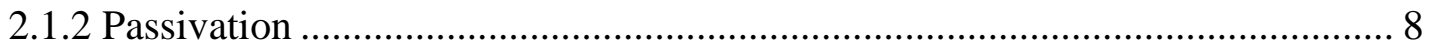

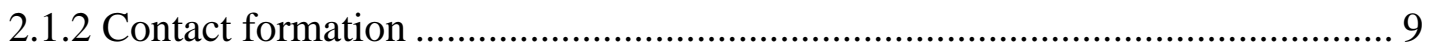

2.2 Fabrication of GaAs/AlGaAs photodiodes ............................................... 11

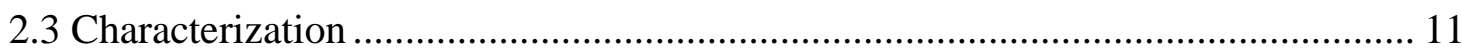

2.3.1 Current-voltage and capacitance-voltage measurements ............................ 11

2.3.2 External quantum efficiency measurements ......................................... 11

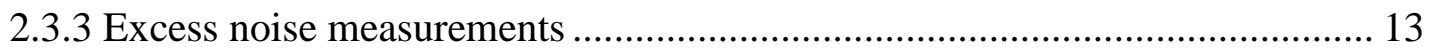

2.3.4 Spatial uniformity measurements ........................................................ 14

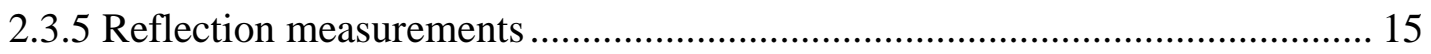


Chapter 3. Deep UV Enhanced 4H-SiC Avalanche Photodiode................................. 17

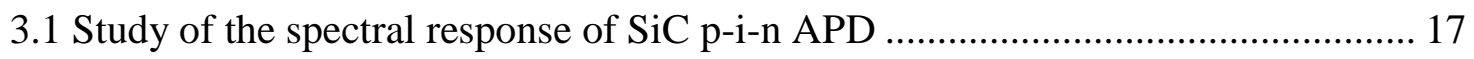

2.1.1 Spectral response modeling and analysis........................................... 17

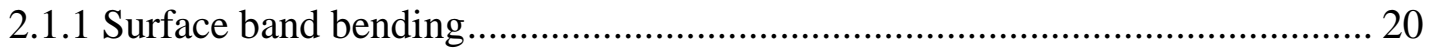

2.1.1 Approaches to enhance the deep-UV response......................................... 22

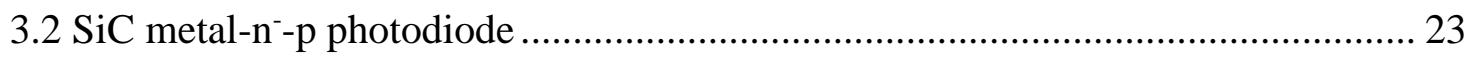

3.3 SiC NIP photodiode with grade doped $n$ layer .............................................. 28

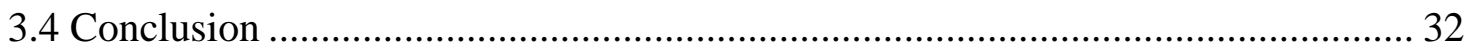

Chapter 4. AlGaN/SiC Photodiode for Deep-UV Detection...................................... 33

4.1 AlGaN/SiC SAM avalanche photodiode .................................................... 33

4.2 $\mathrm{SiC}$ avalanche photodiode with $\mathrm{AlGaN}$ window layer ...................................... 38

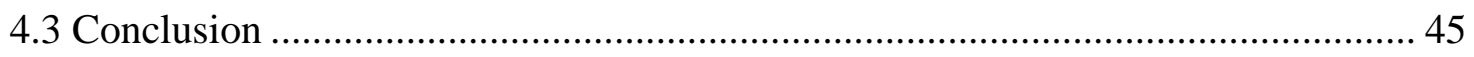

Chapter 5. Alo.8Ga0.2As Avalanche Photodiode for Near-UV Detection.................... 47

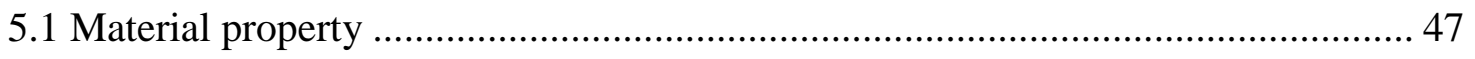

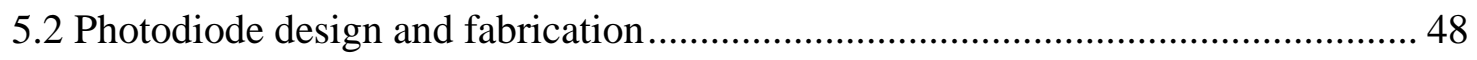

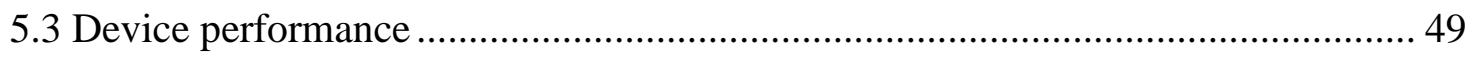

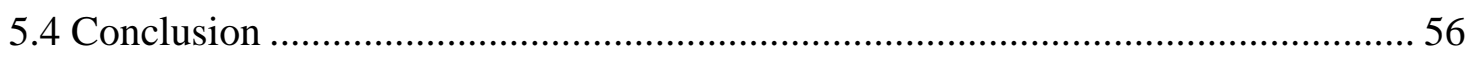

Chapter 6. GaAs/AlGaAs Photodiode for Wide-Spectrum Detection ......................... 57

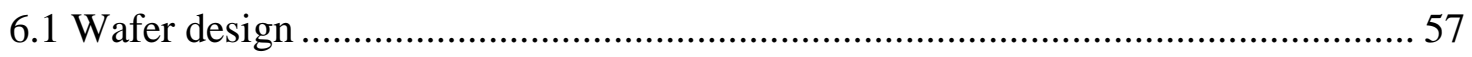

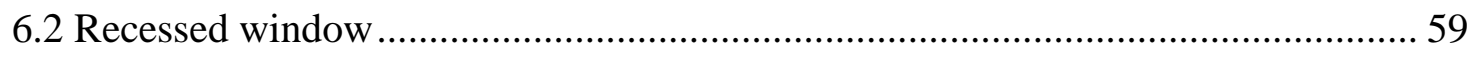

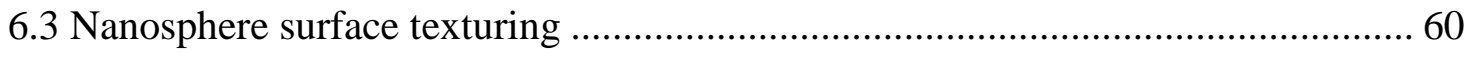

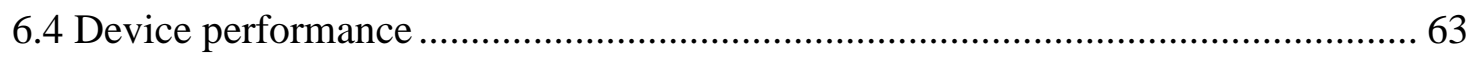

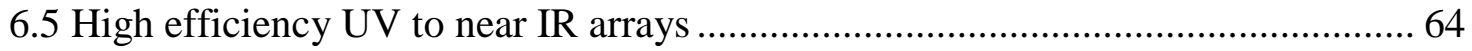




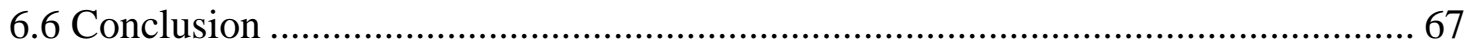

Chapter 7. Conclusion and Future Work ......................................................................... 68

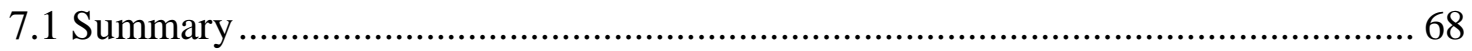

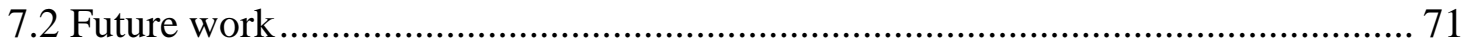

7.2.1 Nitrogen surface passivation on $\mathrm{SiC}$ avalanche photodiode......................... 71

7.2.2 InGaP/AlGaAs UV-enhanced avalanche photodiode ................................. 73 


\section{Table of Figures}

Figure 1-1. Structure of SiC PIN APD with recess window ......................................... 7

Figure 1-2. Measured external quantum efficiency of SiC PIN APD ............................. 4

Figure 3-1. Measured and Simulated Quantum Efficiency of SiC APD ........................ 18

Figure 3-2. Different layers' contribution to quantum efficiency of SiC APD ............... 19

Figure 3-3. Band diagram showing surface band-bending (top) and the spatial distribution

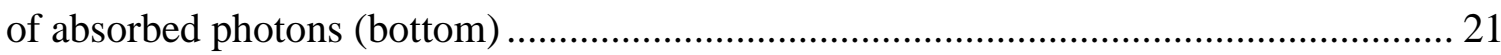

Figure 3-4. Device structure of the SiC metal-n--p photodiode. .................................... 24

Figure 3-5. Transmission of the semitransparent contact. ........................................... 26

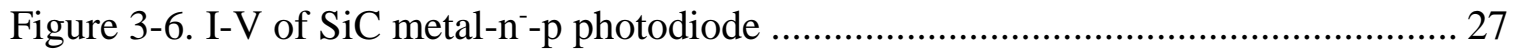

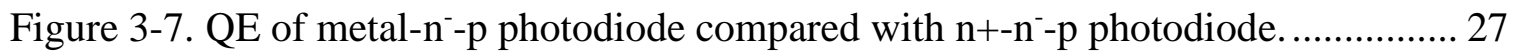

Figure 3-8. Cross section of the SiC NIP photodiode design ................................... 28

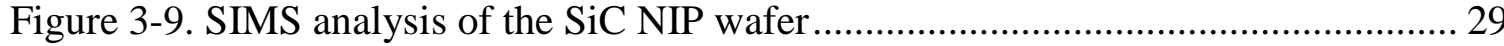

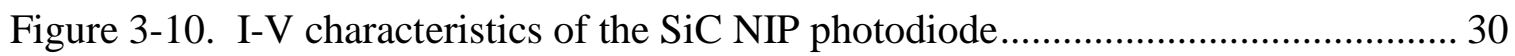

Figure 3-11. External quantum efficiency of the SiC NIP APD with graded $n$ layer....... 31

Figure 4-1. Comparison of a) thick i-layer PIN and b) SACM structure. ........................ 36

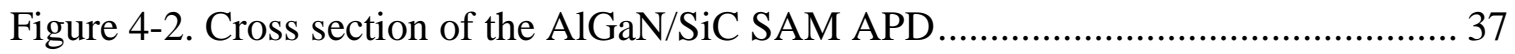

Figure 4-3. External quantum efficiency of AlGaN/SiC SAM APD without (a) and with

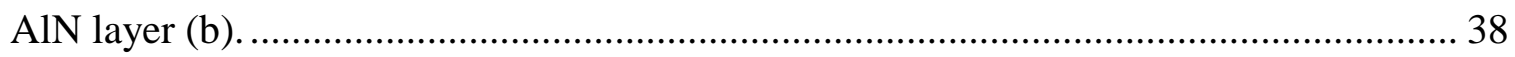

Figure 4-4. Cross section of AlGaN /AlN/SiC photodiode. ......................................... 40

Figure 4-5. External quantum efficiency of AlGaN /AlN/SiC photodiode. .................. 41

Figure 4-6. Band diagram of SiC/AIN/AlGaN for different band in $\mathrm{SiC}$....................... 42

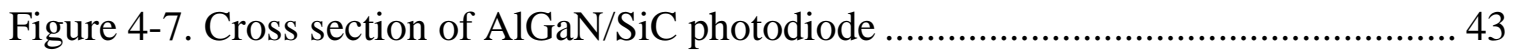


Figure 4-8. Reverse-bias versus gain for $100 \mu \mathrm{m}$ depth recessed window device. 45

Figure 4-9. Band diagram of AlGaN /SiC photodiode. 45

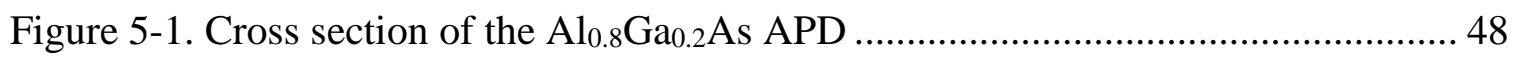

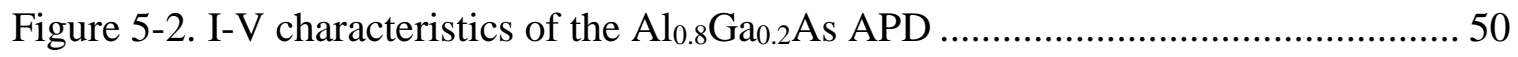

Figure 5-3. Dark current vs device area of $\mathrm{Al}_{0.8} \mathrm{Ga}_{0.2} \mathrm{As}$ APD..................................... 51

Figure 5-4. Measured and simulated external quantum efficiency of $\mathrm{Al}_{0.8} \mathrm{Ga}_{0.2} \mathrm{As}$ APD. 52

Figure 5-5. Capacitance and depletion width vs voltage of the $\mathrm{Al}_{0.8} \mathrm{Ga}_{0.2} \mathrm{As}$ APD ......... 53

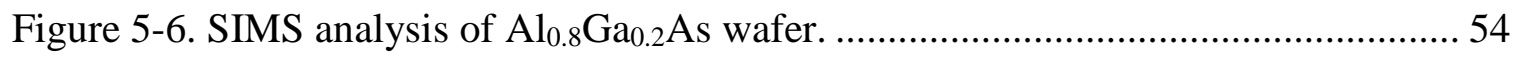

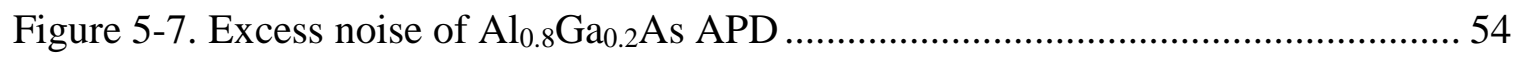

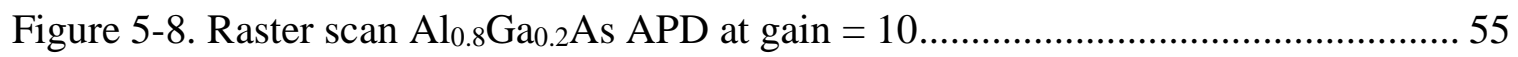

Figure 6-1. Wafer structure for GaAs/AlGaAs photodiode ....................................... 58

Figure 6-2. Current - voltage characteristics of a $200 \mu \mathrm{m}$-diameter GaAs/AlGaAs

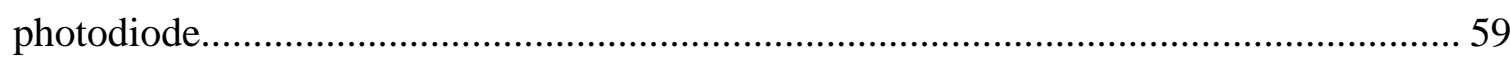

Figure 6-3. SEM image of the recessed window on GaAs/AlGaAs photodiode .............. 60

Figure 6-4. SEM image of the textured surface on GaAs/AlGaAs photodiode................ 62

Figure 6-5. Measured reflectance of the textured surface compared with $\mathrm{SiO}_{2} \mathrm{AR}$ coating

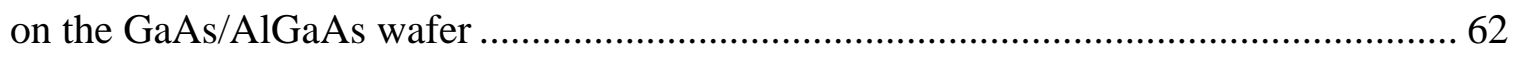

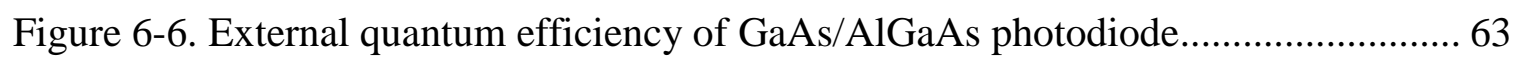

Figure 6-7. (a) Photograph of a 1x16 UV to near IR linear array and (b) enlarged picture

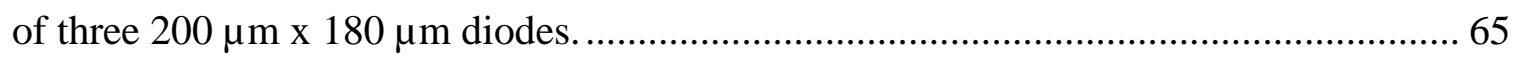

Figure 6-8. Dark current of each element in the 1x16 UV to near IR linear array at $-1 \mathrm{~V}$ bias. 
Figure 6-9. (a) Photograph of a 1x32 UV to near IR linear array and (b) enlarged picture

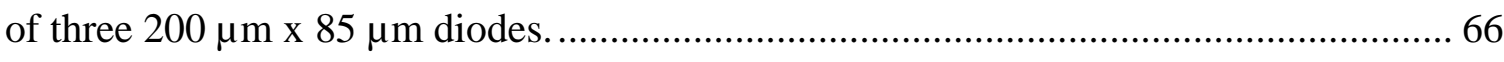
Figure 6-10. Dark current of each element in the $1 \times 32 \mathrm{UV}$ to near IR linear array at $-1 \mathrm{~V}$

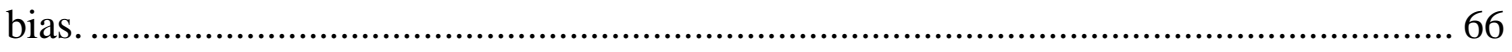

Figure 7-1. Cross section of the InGaP/AlGaAs APD ............................................... 76 


\section{Chapter 1. Introduction}

\subsection{Ultraviolet radiation detection}

The ultraviolet (UV) spectrum, corresponding to photon energies from $3.1 \mathrm{eV}$ to 124 $\mathrm{eV}$ is divided into the following subdivisions [1.1]:

$\begin{array}{lll}\text { Near UV } & \text { NUV } & 400 \mathrm{~nm}-300 \mathrm{~nm} \\ \text { Middle UV } & \text { MUV } & 300 \mathrm{~nm}-200 \mathrm{~nm} \\ \text { Far UV } & \text { FUV } & 200 \mathrm{~nm}-122 \mathrm{~nm} \\ \text { Extreme UV } & \text { EUV } & 122 \mathrm{~nm}-10 \mathrm{~nm}\end{array}$

Ultraviolet light has high photon energy, which can activate many chemical processes. The most important natural UV source is the sun. Approximately $9 \%$ of the energy emitted by the sun is in the UV range. But the stratospheric ozone layer prevents wavelengths shorter than $280 \mathrm{~nm}$ from reaching the Earth's surface. Also UV radiation with wavelength shorter than $200 \mathrm{~nm}$ will be absorbed by oxygen in the atmosphere.

The detection of UV radiation is important for a wide range of civil and military applications, such as chemical and biological analysis (ozone, pollutants, and most organic compounds have absorption lines in the UV range) [1.2], flame detection (fire alarm and missile plume), and short-range secure communication [1.3].

The primary requirements for UV detectors are high quantum efficiency, low dark current, high gain, and low noise. In additions, for some applications, solar-blind operation is very important to eliminate the background noise. Solar-blind operation requires a cutoff in detection for wavelengths longer than $280 \mathrm{~nm}$, since the radiation shorter than 280 is strongly absorbed by the stratospheric ozone layer. 
Traditionally, UV detection has been achieved using photomultiplier tubes (PMTs). They exhibit high sensitivity in the UV and large detection area. However, they are bulky, fragile, subject to magnetic fields, expensive, and they have limited dynamic range. As an alternative, semiconductor photodetectors have the advantages of smaller size and good reliability. For some applications avalanche photodiodes (APDs), which utilize impact ionization to achieve the internal gain, could be a potential replacement for PMTs.

\subsection{Material candidates for UV detection}

Due to its well-established technology, $\mathrm{Si}$ is the most common material choice for APDs for UV detection, although commercial GaAs-based UV photodiodes are also available. However, owing to its narrow bandgap, the peak quantum efficiency of a Si APD is in the visible range. For UV-detection, visible light from the environment will result in a high background noise and expensive filters are required. Also, for high-sensitivity applications, the detector must be cooled to reduce the dark current [1.4].

UV photodetectors based on wide-bandgap semiconductors such as $\mathrm{SiC}$ and III-nitrides have the potential to exceed the performance of Si APDs in some areas [1.5-1.6]. The wide bandgap itself is an important advantage for photodetectors, since it enables roomtemperature operation and provides intrinsic visible-blindness. In addition, the thermal conductivity of wide bandgap materials is, in general, significantly higher than silicon, which makes them suitable for high temperature and high power applications. 


\subsection{Review of SiC PIN-structure APDs}

4H-SiC, with an indirect bandgap of $3.2 \mathrm{eV}$, is particularly suitable for UV detection. Previous students in our group have done extensive studies on 4H-SiC APDs and developed various technologies to improve the performance [1.7-1.9]. State-of-art SiC APDs exhibit extremely low dark current of $0.18 \mu \mathrm{A} / \mathrm{cm}^{2}$ at a gain of 1000. By incorporating a recessed, window a peak external quantum efficiency of $65 \%$ has been achieved [1.10]. This structure is shown in Fig. 1-1. However, the photo response decreases rapidly below $260 \mathrm{~nm}$ and has a long tail out to $380 \mathrm{~nm}$, which limits their use for solar-blind applications. This relatively narrow spectral response limits the applications in which SiC APDs can be deployed. The measured external quantum efficiency of the structure in Figure 1-1 is shown in Figure 1-2.

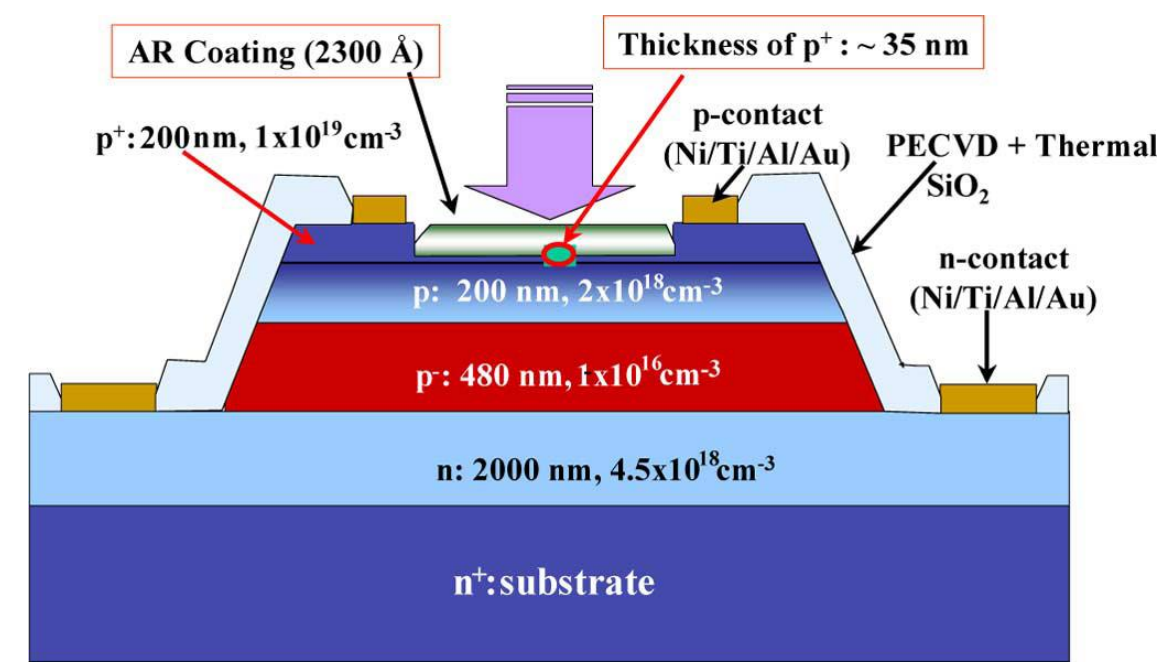

Figure 1-1. Structure of SiC PIN APD with recess window

Beginning from the top, the structure consists of a $200 \mathrm{~nm} \mathrm{p}+$ layer $\left(1.0 \times 10^{19} \mathrm{~cm}^{-3}\right)$, a $200 \mathrm{~nm}$ p layer $\left(2.0 \times 10^{18} \mathrm{~cm}^{-3}\right)$, a $480 \mathrm{~nm} \mathrm{p}^{-}$layer $\left(1.0 \times 10^{16} \mathrm{~cm}^{-3}\right)$ and a $2000 \mathrm{~nm}$ 
$\mathrm{n}$ buffer layer $\left(4.5 \times 10^{18} \mathrm{~cm}^{-3}\right)$. The $\mathrm{p}+$ contact layer was etched from an initial thickness of $200 \mathrm{~nm}$ to about $35 \mathrm{~nm}$ in the central active region to form a recessed window. Also a $230 \mathrm{~nm} \mathrm{SiO}_{2}$ was deposited as an anti-reflection coating (optimized for $260 \mathrm{~nm}$ ).

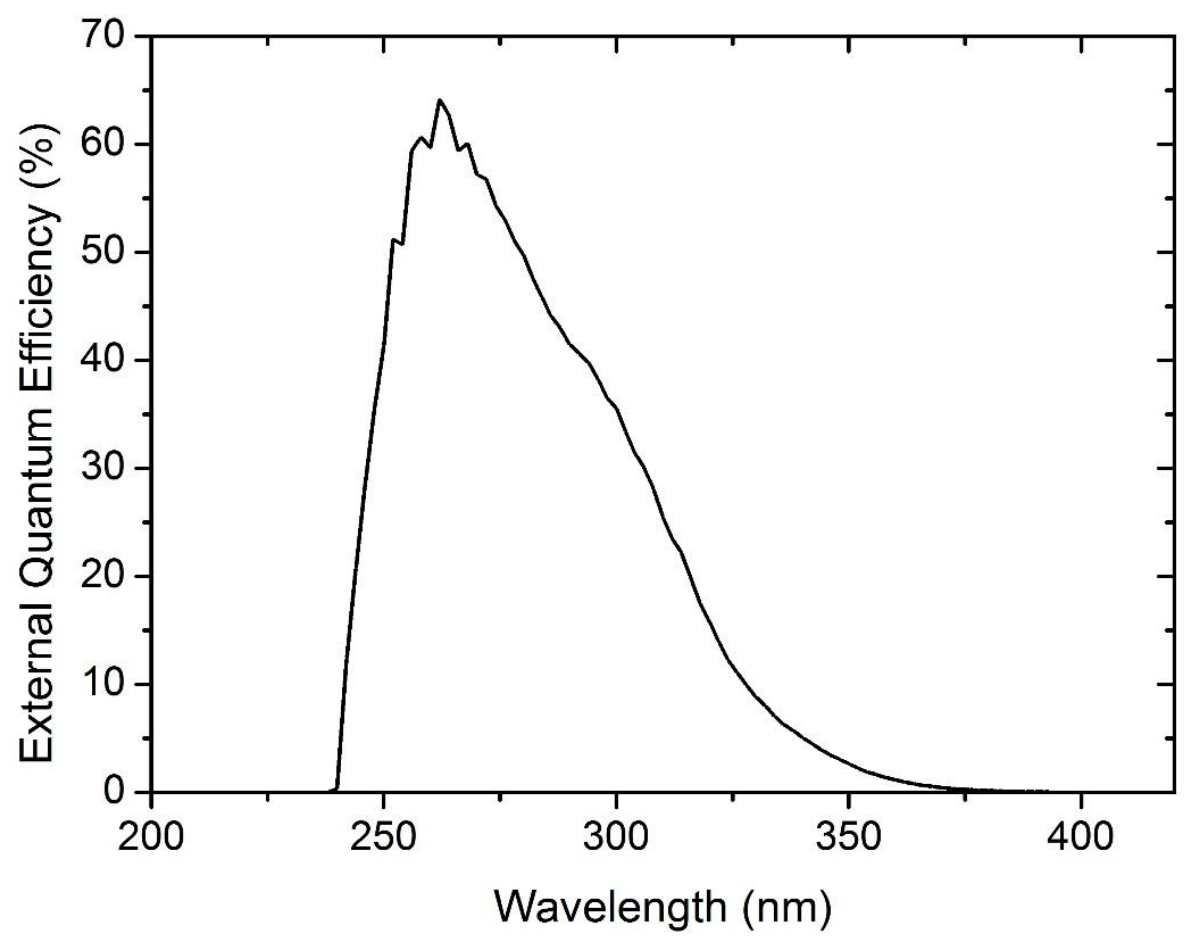

Figure 1-2. Measured external quantum efficiency of SiC PIN APD

My work focused on developing new photodetectors with high efficiency in the spectral regions where current $\mathrm{SiC}$ photodiodes have poor response. The discussion will be divided into two parts, the deep-UV $(200 \mathrm{~nm}-250 \mathrm{~nm})$ and the near-UV $(300 \mathrm{~nm}-400 \mathrm{~nm})$. The work in the deep-UV will be SiC-based and the work in the near-UV spectrum will utilize GaAs and AlGaAs. 


\subsection{Organization of dissertation}

This dissertation is divided into 7 chapters. In chapter 1, I have briefly introduced the background of UV detectors and the performance of SiC PIN APDs. In chapter 2, I describe the general process of fabricating AlGaN/SiC- and GaAs/AlGaAs-based APDs and present the experimental characterization techniques for photodiodes. Chapter 3 focuses on the different approaches that I have investigated to improve the deep-UV response of $4 \mathrm{H}-\mathrm{SiC}$ detectors. In chapter 4, $\mathrm{AlGaN} / \mathrm{SiC}$ heterogenous structure photodiodes are demonstrated as an alternative way to achieve good deep-UV detection. Both a separate absorption and multiplication (SAM) structure and a transparent window structure were studied. In chapter 5, $\mathrm{Al}_{0.8} \mathrm{Ga}_{0.2} \mathrm{As}$, as a material candidate to replace $\mathrm{SiC}$ and $\mathrm{Si}$ for near-UV detection, is described. In Chapter 6 I discuss my work using a recessed window structure combined with a surface texturing technique to achieve high quantum efficiency from near-UV to near-IR in GaAs detectors. Chapter 7 concludes my work and discusses possible future research directions closely related to this work. 


\section{Chapter 2. Fabrication and characterization of photodetectors}

The first part of this chapter will discuss fabrication of III-nitride/SiC photodiodes and GaAs/AlGaAs photodiodes. Since these two material systems are very different in their fabrication processes, they will be discussed separately. In the later part of this chapter I describe several of the measurement setups used to characterize the devices developed in this work.

\subsection{Fabrication of AlGaN/SiC Photodiodes}

\subsubsection{Sample preparation}

SiC wafers purchased from Cree Inc. are often four-inch double-side-polished wafers. Usually there will be markers on the back side of the wafer. But sometimes there are no markers on either side of the wafer. In this case, a standard sheet resistance measurement using a four-point probe setup is used to determine on which side the epitaxial layers were grown. The resistivity of the epitaxial layer is usually several orders of magnitude higher than the highly doped substrate. For the $\mathrm{AlGaN} / \mathrm{SiC}$ sample, the epitaxial layer side can be determined from the color.

After this step, the wafer is scored with a diamond scribe in order to cleave it into small

pieces. The relative locations of the small pieces are recorded. Then one of the pieces is cleaned using the following procedure: First the sample is submerged in an ultrasonic cleaner for 5 minutes, and then rinsed with methanol and de-ionized (DI) water. After cleaning, the sample is blown dry using a nitrogen gun and baked at $90{ }^{\circ} \mathrm{C}$ on a hot plate 
for 1 minute to make sure the water is fully evaporated. This step is necessary since water will affect adhesion of photoresist in the following steps.

\subsubsection{Mesa patterning}

Both $\mathrm{SiC}$ and III-Nitride are very hard materials, which makes it difficult to pattern them. No standard wet etch technique with acceptable etching rate at room temperature is available. So dry etching using reactive ion etch (RIE) and inductively coupled plasma (ICP) etch were used.

Depending on the required etch depth, different types of photoresist can be used to pattern mesas. Usually, AZ4620, a thick photoresist, is used to achieve etch depths deeper than $1 \mu \mathrm{m}$. Prior to coating with photoresist, Hexamethyldisiloxane (HMDS), an adhesion promoter for photoresist, is dropped on the wafer and spun at 4000 revolutions per minute (rpm) for $30 \mathrm{~s}$. Next AZ 4260 photoresist is applied to the wafer and spun at $4000 \mathrm{rpm}$ for $40 \mathrm{~s}$. The wafer is then baked for $3 \frac{1 / 2}{2}$ minutes at $90^{\circ} \mathrm{C}$. Then the sample is exposed with a Suss MicroTech MJB-4 aligner using the "mesa" mask. The exposure time is different for different photomask materials, e.g., 160 seconds is used for a quartz mask and 250 seconds is used for soda lime glass mask. The exposed photoresist is then developed in a solution of AZ 400K diluted to a ratio1:3 AZ 400K: de-ionized water. The total time for this step can range from $40 \mathrm{~s}$ to $1 \mathrm{~min}$. Following confirmation that the photoresist has been completely developed by observing under a microscope, the wafer is baked again at 130 ${ }^{\circ} \mathrm{C}$ for five minutes. This will make the photoresist reflow and harden, so that a small bevel angle at the edge of the photoresist is formed. This step is critical for the AlGaN/SiC mesa 
etch, because this bevel angle will be transferred to the device during the dry etch, which lowers the electric field at the sidewall. The profile of the patterned photoresist is measured with a profile meter. Typically the thickness of the photoresist is $\sim 7 \mu \mathrm{m}$ and the bevel angle is less than 20 degrees.

The mesa is etched by inductively coupled plasma (ICP) with an Oxford ICP 100 system using the following recipe. RF power $160 \mathrm{~W}$, ICP power $1000 \mathrm{~W}$, and pressure of $6 \mathrm{mTorr}$ using $10 \mathrm{sccm}$ Ar and $40 \mathrm{sccm} \mathrm{Cl}_{2}$ at $-25^{\circ} \mathrm{C}$. A small drop of fomblin oil is placed on a silicon wafer carrier with a thick $\mathrm{SiO}_{2}$ layer; the wafer is placed on top of the oil. This prevents the wafer from overheating during the etch process, which can result in uneven etching or difficulty removing the photoresist. The etch rate is approximately $5 \mathrm{~nm} / \mathrm{s}$ for $\mathrm{SiC}$, and 10 to $20 \mathrm{~nm} / \mathrm{s}$ for AlGaN. The etching selectivity of $\mathrm{SiC}$ relative to photoresist is approximately 1:3. After etching, the photoresist is removed by ultrasonic cleaning in acetone. An optional oxygen plasma with power 200 to $300 \mathrm{~W}$ can be used if the acetone clean does not work well. This is followed by a spin clean with acetone, methanol, and deionized water.

\subsubsection{Passivation}

In order to remove ICP etch residues, the wafer is first immersed in $25 \%$ ammonium hydroxide in an ultra-sonic bath for 10 minutes. Then a modified RCA cleaning process is performed. The sample is first dipped in 1:1:4 mixture of $\mathrm{NH}_{4} \mathrm{OH}, \mathrm{H}_{2} \mathrm{O}_{2}$, and DI water at $75^{\circ} \mathrm{C}$ for 5 minutes to clean the organic residues. Then it is dipped in buffered oxide etchant (BOE) for 1 minute to remove native oxides and some of the ionic contaminants. Finally 
the sample is dipped in 1:1:4 mixture of $\mathrm{HCl}, \mathrm{H}_{2} \mathrm{O}_{2}$, and DI water at $75^{\circ} \mathrm{C}$ for 5 minutes to remove the remaining traces of ionic contaminants. If the wafer has an AlGaN layer, the sample is loaded onto the heated shelf of the March oxygen plasma cleaner immediately after the RCA clean to perform thermal oxidation. An oxidation of 15 minutes followed by immersion in BOE for 1 minute is repeated 3 times to remove the surface damage. The thin layer of oxide from the last oxidation is kept as a passivation layer. If the wafer is pure $\mathrm{SiC}$, an oxidation in the furnace at higher temperature is preferred. Instead of loading the wafer into an oxygen plasma cleaner, the wafer is placed in a quartz boat and loaded into the center in the oxidation furnace. A thermal oxidation at $1050{ }^{\circ} \mathrm{C}$ for 3.5 hours is used to form a thin silicon oxide passivation layer. This is followed by $\mathrm{SiO}_{2}$ deposition by plasma enhanced chemical vapor deposition (PECVD) to achieve the final anti-reflection coating thickness. An $\mathrm{SiO}_{2}$ layer is then deposited by PECVD using the following recipe: temperature $=285^{\circ} \mathrm{C}, \mathrm{RF}$ power $=33$, reflected $\mathrm{RF}$ power $=0$, pressure $=800 \mathrm{mTorr}$ and gases; $105 \mathrm{sccm} \mathrm{SiH}_{4}, 400 \mathrm{sccm} \mathrm{N}_{2} \mathrm{O}$ and $500 \mathrm{sccm} \mathrm{N}$. The rate of deposition is approximately $10 \mathrm{~nm} / \mathrm{min}$. The same recipe is used for $\mathrm{SiO}_{2}$ deposition for all fabrication described in this dissertation. Based on the reflectance calculations, a specific thickness is used to create an anti-reflection coating optimized at a desired wavelength.

\subsubsection{Contact formation}

AZ nLOF 2020 negative photoresist is used to define the pattern for contact formation. The photoresist is applied to the sample and spun at $5.5 \mathrm{krpm}$ for 30 seconds and then baked at $110{ }^{\circ} \mathrm{C}$ on a hotplate for 1 minite. The pattern is defined by exposure for 1.5 
seconds using the "contact" mask. This is followed by 1 minute post-exposure bake at 110 ${ }^{\circ} \mathrm{C}$ and developing in AZ $300 \mathrm{MIF}$ for about 30 seconds. Oxygen plasma etch using a March plasma cleaner at power $150 \mathrm{~W}$ for $1 \mathrm{~min}$ is used to remove the undeveloped photoresist and ensure that the contact window is open. A chemical etch in $\mathrm{BOE}$ removes the $\mathrm{SiO}_{2}$ in the contact window for access to the semiconductor. The etch rate is approximately 110 $\mathrm{nm} / \mathrm{min}$; over etching by 15 seconds ensures all $\mathrm{SiO}_{2}$ is removed. The wafer is rinsed thoroughly in de-ionized water and blown dry with the nitrogen gun. The wafer is then placed in the March plasma cleaner again for oxygen plasma etch at the same power and time. This etch is to remove any residue left behind following the $\mathrm{SiO}_{2}$ removal and it also ensures a clean surface for metal deposition.

The wafer is then loaded into the electron beam evaporator. Four layers of metal are deposited for both $\mathrm{p}$ and $\mathrm{n}$ contacts on $\mathrm{SiC}$ in the following sequence: $\mathrm{Ni} 25 \mathrm{~nm} / \mathrm{Ti} 35 \mathrm{~nm}$ / Al $100 \mathrm{~nm} / \mathrm{Ni} 80 \mathrm{~nm}$. For the n contact on AlGaN, V 15 nm / Al 80 nm / V $20 \mathrm{~nm} / \mathrm{Au}$ $100 \mathrm{~nm}$ is used. The photoresist and unnecessary metal is removed by metal lift-off. Initially the wafer is soaked in acetone for 30 mins and then light ultra-sonic agitation is used to remove any remaining metal. The wafer is spin cleaned with acetone, methanol, DI water and dried. A contact anneal by rapid thermal anneal (RTA) for 3 minutes at $850{ }^{\circ} \mathrm{C}$ in vacuum is then performed to achieve ohmic contacts and lower the contact resistance.

If no contact pad is required the device is ready for testing. If a contact pad is required nLOF 2020 is again used to pattern the contact pad. A two layer metal stack is used for the contact pad: Ti $20 \mathrm{~nm} / \mathrm{Au} 150 \mathrm{~nm}$. Metal lift-off completes the fabrication and the devices are ready for testing. 


\subsection{Fabrication of GaAs/AIGaAs Photodiodes}

There are some similarities between the fabrication steps for III-nitride/SiC and GaAs/AlGaAs photodiodes. Therefore I will only discuss the steps that are unique to the GaAs/AlGaAs photodiode fabrication process. The photolithography process for mesa definition is similar, but instead of using AZ4620, a thinner photoresist AZ5214 is used. A 1:1:10 mixture of $\mathrm{H}_{3} \mathrm{PO}_{4}, \mathrm{H}_{2} \mathrm{O}_{2}$ and DI water is used to etch the mesas. The etch rate for GaAs is about $300 \mathrm{~nm} / \mathrm{s}$. For AlGaAs, the etch rate will increase with $\mathrm{Al}$ mole fraction. The typical etch rate for $\mathrm{Al}_{0.8} \mathrm{Ga}_{0.2} \mathrm{As}$ is $1000 \mathrm{~nm} / \mathrm{s}$.

The mesa etch is followed by sulfur sidewall passivation. For this procedure, the wafer is soaked in 5\% ammonium sulfide solution for 5 minutes and then rinsed with DI water and blown dry using a nitrogen gun. After that, a $300 \mathrm{~nm}$-thick layer of $\mathrm{SiO}_{2}$ is deposited by PECVD to isolate the passivated sidewall from the air. The contact window is formed in the same manner as above but the metal contact stack is Ti $30 \mathrm{~nm} / \mathrm{Pt} 50 \mathrm{~nm} / \mathrm{Au} 100$ $\mathrm{nm}$. The wafer is then annealed using RTA at $450{ }^{\circ} \mathrm{C}$ for 30 seconds in nitrogen. All the other steps are the same as the fabrication of $\mathrm{AlGaN} / \mathrm{SiC}$ photodiodes.

\subsection{Characterization}

The following characterization techniques are used to determine photodiode and avalanche photodiode performance. 


\subsubsection{Current-voltage and capacitance-voltage measurements}

DC current-voltage (I-V) measurements are made using a low-noise probe station capable of low current measurement (as low as 1fA with sufficient integration time) and an HP 4156B semiconductor parameter analyzer. A $30 \mathrm{~W}$ deuterium lamp is used as a broad spectrum UV light source for the photocurrent measurement. Photocurrent and dark current are measured versus bias voltage. Avalanche gain at a particular voltage is calculated using the flowing formula:

$$
\mathrm{M}=\frac{I_{\text {photo }}-I_{\text {dark }}}{I_{\text {photo_unity }}-I_{\text {dark_unity }}}
$$

where $I_{\text {photo }}$ and $I_{\text {dark }}$ are the photocurrent and dark current, respectively. $I_{\text {photo_unity }}$ and $\mathrm{I}_{\text {dark_unity }}$ are the photocurrent and dark current at a predetermined bias where the avalanche gain is insignificant. A bias that is $\sim 50 \%$ of the breakdown voltage is usually used as the unity gain point for a PIN structure.

The capacitance-voltage $(\mathrm{C}-\mathrm{V})$ measurement utilizes the same probe station. The semiconductor parameter analyzer is replaced by an HP 4275A multi-frequency LCR meter. Open-circuit and short-circuit calibrations are performed prior to measurements. The capacitance-voltage measurement is useful in determining the reach-through voltage of a separate absorption and multiplication (SAM) structure APD. It can also be used to determine the depletion width of photodiodes.

\subsubsection{External quantum efficiency measurements}

The external quantum efficiency is the ratio of the number of carriers collected to the number of photons incident on the photodiode at unity gain. In some publications, 
responsivity is used instead of quantum efficiency. Responsivity, which has a unit of amperes per watt, is the unity-gain photocurrent per incident power at a given wavelength from the photodiode. The responsivity can be calculated from quantum efficiency by the following formula:

$$
R_{\lambda}=\eta_{\lambda} \lambda \cdot \frac{q}{h c} \approx \eta_{\lambda} \lambda \cdot \frac{1}{1240 \mathrm{~W} \cdot \mathrm{nm} / \mathrm{A}}
$$

where $\lambda$ is the wavelength, $\eta_{\lambda}$ is is the quantum efficiency at given wavelength, $\mathrm{q}$ is the elementary charge, $\mathrm{c}$ is the speed of light in vacuum, h is Planck's constant, and $\mathrm{R}$ is the responsivity.

A laser driven light source, which has significantly higher power in the UV spectrum than other wide spectrum light sources such as a Xenon lamp is used in our quantum efficiency setup. The light from the light source is coupled into a monochromator, which functions as a tunable, narrow spectral width source from the UV to the visible. The original blazed grating in the spectrometer, which has a blaze wavelength of $1 \mu \mathrm{m}$ was replaced by a new blazed grating with a blaze wavelength of $250 \mathrm{~nm}$ to further enhance the UV light power from the spectrometer. The output from the monochromator is coupled into a $300 \mu \mathrm{m}$-core UV enhanced fiber that has low attenuation in the UV. From the fiber output two uncoated microscope objectives placed back-to-back collimate the beam and focus the light to a small spot on the active area of the device. It is important that all the light is focused on the device in order to get an accurate measurement. A chopper placed in front of the fiber input provides frequency modulation to the signal (usually $200-300$ Hz). A Stanford Research SR-850 lock-in amplifier measures the photocurrent of the device. The photocurrent response of a calibrated UV-enhanced silicon detector is also 
measured. The quantum efficiency and responsivity of the device under test (DUT) can then be calculated from the following formula:

$$
\eta_{D U T}=\eta_{r e f} \cdot \frac{I_{D U T}}{I_{r e f}}
$$

where $\eta_{r e f}$ is the quantum efficiency of the calibrated silicon detector. IDUT and $\mathrm{I}_{\text {ref }}$ are the test device and reference photocurrents.

\subsubsection{Excess noise measurements}

The excess noise measurement characterizes the noise that arises from fluctuations in the gain of an avalanche photodiode. Avalanche gain results from impact ionization of the carriers. The carriers gain energy from the high electric field and initiate impact ionization. However, impact ionization is a stochastic process. Not all the carriers experience the same gain. Excess noise can be characterized by the excess noise factor $\mathrm{F}(\mathrm{M})$.

The excess noise factor is measured by a HP8970B noise figure analyzer. An Ar-ion laser that operates at $351 / 363 \mathrm{~nm}$ is used as the light source. The device is biased by a Keithley 2400 source meter through a bias tee and the AC component is fed into the noise figure analyzer. The center frequency of the HP8970 is chosen so that ambient noise is minimized.

The excess noise figure, $\mathrm{F}(\mathrm{M})$, of an APD can be calculated from the spectral noise density, $S$, where $S$ is given by Eqn. 2-1 below. In the equation $I_{0}$ is the photocurrent at unit gain, $R(\omega)$ is the frequency dependent impedance of the system and $M$ is the gain. 
Eqn. 2-1

The gain can be calculated based on the DC current read from the Keithley source meter. $2 \mathrm{qR}(\omega)$ is determined by measuring the noise versus different photocurrent, $\mathrm{I}_{0}$, at unity gain. Also, the effective impact ionization coefficient ratio $\mathrm{k}$ is can be calculated using McIntyre's local mode equation [2.1]:

$$
\mathrm{F}(\mathrm{M})=\mathrm{kM}+(1-\mathrm{k})(2-1 / \mathrm{M}) \text {. }
$$

Eqn. 2-2

\subsubsection{Spatial uniformity measurements}

A spatial uniformity scan provides a picture of the spatial response of the detector, which is useful to determine if there are "hot spots" such as edge breakdown or microplasmas. It can also reveal low response in the active area due to high lateral resistance. For this measurement, the chopped UV light from the Ar-ion laser is focused onto the APD by a UV lens with a spot size less than $5 \mu \mathrm{m}$. The lens is mounted on a motorized micropositioner that can be controlled by a computer. A labview program controls the movement of the micropositioner and collects photocurrent from the lock-in amplifier.

\subsubsection{Surface Reflection}

Owing to the significant refractive index difference between the semiconductor and air, part of the incident optical signal is reflected, a factor that limits the quantum efficiency. Therefore it is important to minimize the light reflection at the operating wavelength. The 
reflectance is characterized with a Perkin Elmer Lambda 950 spectrometer. The measurement steps are similar to the quantum efficiency measurement. First the optical power reflected by the semiconductor wafer is measured. Then a calibrated mirror with known reflectance is measured under the same conditions. The reflectance of the sample can be calculated by multiplying the reflectance reading from the spectrometer by the reflectance of the calibrated mirror. 


\section{Chapter 3. Deep-UV Enhanced 4H-SiC Avalanche Photodiode}

As discussed in the first chapter, $4 \mathrm{H}-\mathrm{SiC}$ is very suitable for UV detection. The wide bandgap results in very low dark current. It also has a favorable impact ionization coefficient ratio. High performance $\mathrm{SiC}$ APDs with high peak responsivity, low dark current, low excess noise, and high gain with diameters up to $250 \mu \mathrm{m}$ have previously been demonstrated by our group. However, the narrow spectral response limited its applications in deep-UV detection. In this chapter, I discuss the source of the poor deep-UV response of SiC PIN APD and different approaches to enhance the deep-UV performance.

\subsection{Spectral response study of SiC PIN APD}

\subsubsection{Spectral response modeling and analysis}

In order to better understand the spectral response curve that is shown in Figure 1-2, the quantum efficiency of the $4 \mathrm{H}-\mathrm{SiC}$ APD with PIN structure shown in Figure 1-1 is modeled using a simple method that is described in Ref [3.1]. The idea of the modeling is that for the PIN structure, the total photocurrent measured externally is due to i) electrons collected from the $\mathrm{p}$ layer at the $\mathrm{p}$-i depletion edge, ii) electron-hole pairs collected from within the depletion region, and iii) holes collected from the n layer at the i-n depletion edge. It is assumed that all carriers generated within the depletion region are collected, while only a fraction of the carriers generated in the undepleted $\mathrm{p}$ and $\mathrm{n}$ regions are 
collected. The collection efficiency depends on the layer thickness, diffusion length, and surface recombination velocity.

The modeled spectral response was fitted to the measured data by adjusting the fitting parameters. The fitted external quantum efficiency curve and the measured curve are shown Figure 3-1.

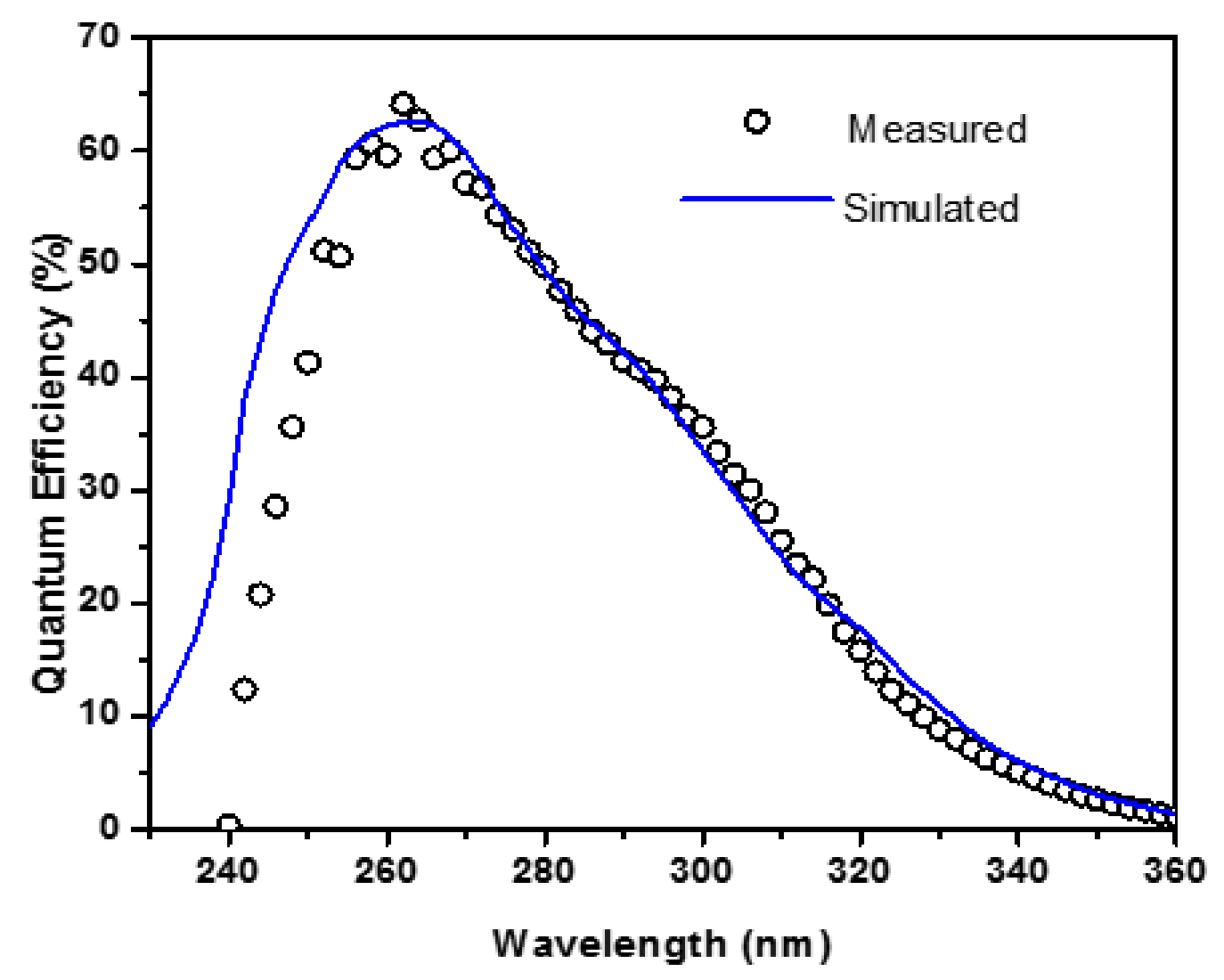

Figure 3-1. Measured and Simulated Quantum Efficiency of SiC APD

To better illustrate this, the contribution of different layers to the external quantum efficiency is compared in Figure 3-2 at three typical wavelengths $(240 \mathrm{~nm}, 265 \mathrm{~nm}$, and $320 \mathrm{~nm}$ ). Since $\mathrm{SiC}$ has an indirect bandgap, the absorption near the band edge is very weak. Therefore at $320 \mathrm{~nm}$, most of the photons are absorbed in the $\mathrm{n}$ buffer layer and the 
substrate. Due to the short diffusion length in the highly doped material, many of the carriers are absorbed in the $\mathrm{n}$ buffer layer and the substrate. Owing to the short diffusion length in the highly doped material, many of the carriers recombine before reaching depletion region. The absorption profile was optimized for operation at $265 \mathrm{~nm}$, so that most of these carriers are absorbed in the depletion region and, thus, high quantum efficiency was achieved.

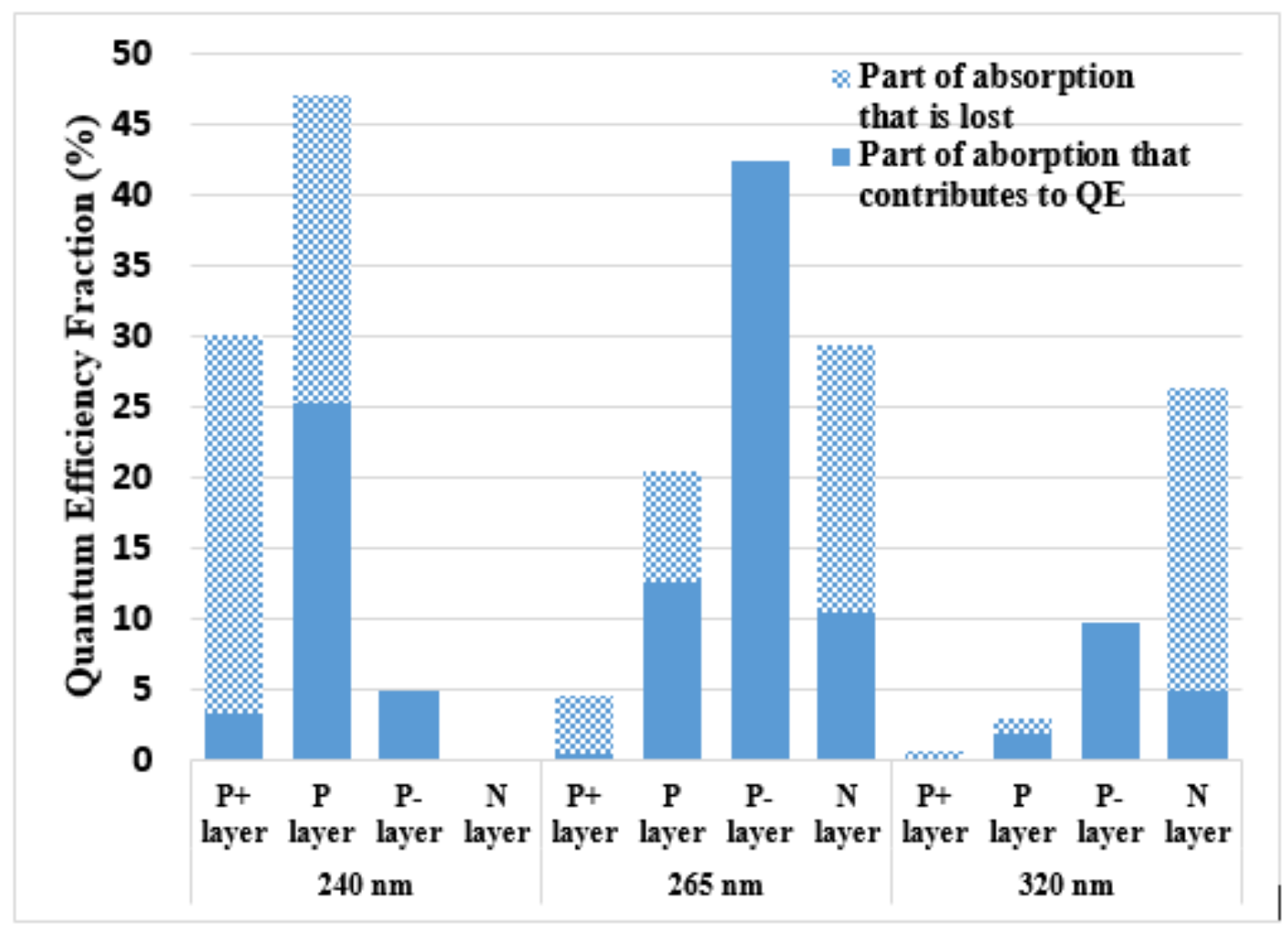

Figure 3-2. Contribution of different layers to quantum efficiency for incident wavelengths of $240 \mathrm{~nm}, 265 \mathrm{~nm}$, and $320 \mathrm{~nm}$.

At $240 \mathrm{~nm}$, most of the photons are absorbed in the top two layers. Similar to the carriers generated in the $\mathrm{n}$ layer, the photons generated in the highly doped $\mathrm{p}$ region also have short diffusion lengths. But there is another effect that also limits the deep UV 
response, surface recombination. Surface recombination is characterized by the surface recombination velocity.

\subsubsection{Surface band-bending}

Surface recombination is caused by band bending at the surface [3.2]. This is illustrated in Figure 3-3. Surface defects such as dangling bonds, pin the Fermi level near the middle of the band gap at the surface. This creates a region where photo generated carriers are pushed toward the surface where they quickly recombine through trap states instead of diffusing to the depletion region where they contribute to the photocurrent and are collected. Surface recombination is characterized by the surface recombination velocity. The reported surface recombination velocity of $\mathrm{SiC} \sim 1 \times 10^{8} \mathrm{~cm} / \mathrm{s}$ [3.3], which is about 6 orders of magnitude higher than that of $\mathrm{Si}[3.4]$. 


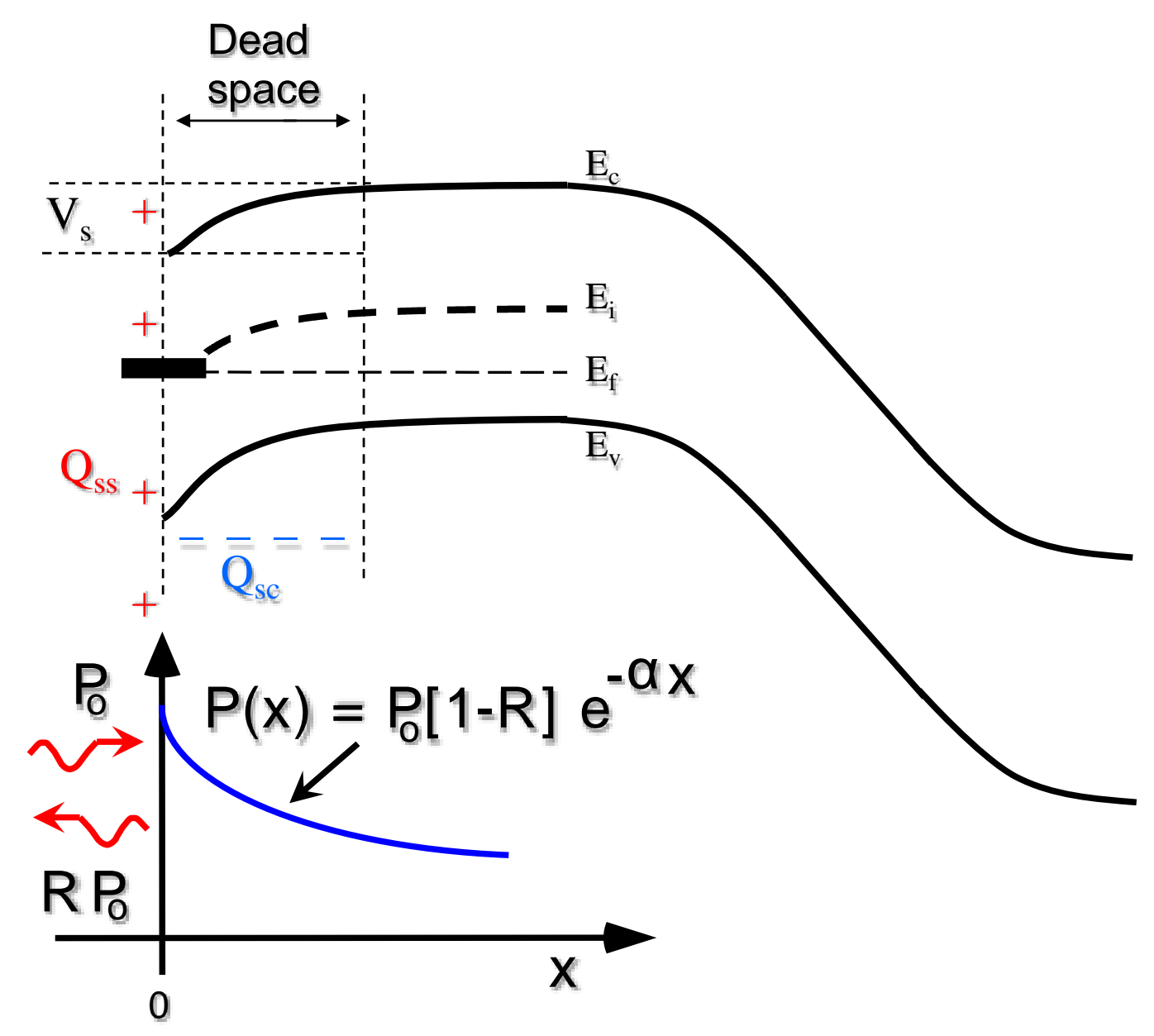

Figure 3-1. Band diagram showing surface band-bending (top) and the spatial distribution of absorbed photons (bottom)

In summary, $\mathrm{SiC}$ has a narrow spectral response. The poor response at wavelengths shorter than $260 \mathrm{~nm}$ is due to the shallow absorption, the high surface recombination velocity, and short diffusion length. The slowly decreasing response at wavelength longer than $300 \mathrm{~nm}$ is due to fact that the absorption is weak at the band edge for the indirect bandgap material. 


\subsubsection{Approaches to enhance the deep-UV response of SiC photodiode}

From the study of the spectral response of the SiC PIN APDs, we know that the deepUV response is greatly affected by three parameters: top doped layer thickness, diffusion length, and surface recombination velocity.

Firstly, a thinner top highly-doped layer will reduce the percentage of photons that are absorbed in this layer, which will minimize the loss of the photon-generated carriers due to the short diffusion length and high surface recombination velocity. Usually this is accomplished using a recessed window structure, which has already been studied by previous students in our group and proved to be effective if the recessed depth is optimized. Theoretically greater deep-UV enhancement will be achieved with deeper recessed windows. But as the top highly-doped layer becomes thinner, the lateral resistance will increase to the extent of causing non-uniform response across the active area. In order to overcome this problem, a thin layer of semitransparent metal can be deposited on the active area of the photodiode. A metal- $n^{-}-\mathrm{p}$ structure utilizing this approach is discussed below in 3.2 .

Secondly, it will also be beneficial to increase the diffusion length. However, since the diffusion length strongly depends on material quality and the doping level, modifying it is not straightforward. Instead of increasing the diffusion length, a high-low junction can be incorporated into the highly-doped layer to provide an effective built-in electric field. This electric field can assist drift into the depletion region and increase efficiency. Also the electric field created by the high-low junction near the top surface will block carriers from reaching the top surface, thus suppressing surface recombination. This approach is 
described in section 3.3

Since high surface recombination velocity is the most detrimental factor for the deepUV performance of SiC, I have also analyzed surface band bending and found that it is due primarily to the dangling bonds that arise from termination of the periodic crystal structure. There are two ways to solve this problem: a) Use a transparent window layer on top of the absorption material. This is widely used in InP/InGaAs photodiodes in which InP acts as transparent window layer at telecom wavelengths [3.5]. A similar structure with AlGaN acting as window layer on $\mathrm{SiC}$ is discussed in chapter 4. b) Choose suitable material to passivate the top surface in order to lower the surface state density. For example, sulfur is a good passivation material on GaAs [3.6] and hydrogen is found to be effective on $\mathrm{Si}$ [3.7]. Similar surface passivation treatment on $\mathrm{SiC}$ discussed in chapter 7 .

\section{2 $\mathrm{SiC}$ metal-n"-p photodiodes}

From the analysis of the photo-response of $\mathrm{SiC}$ in the last section, we know that if most of the photons are absorbed in the depletion region, high quantum efficiency can be achieved. Therefore different Schottky [3.8] and metal-semiconductor-metal (MSM) structures [3.9] have been studied by other groups. As an alternate approach, we examined $\mathrm{SiC}$ a metal-n-p structure, where the $\mathrm{n}-$ absorption region is fully depleted at low bias. 


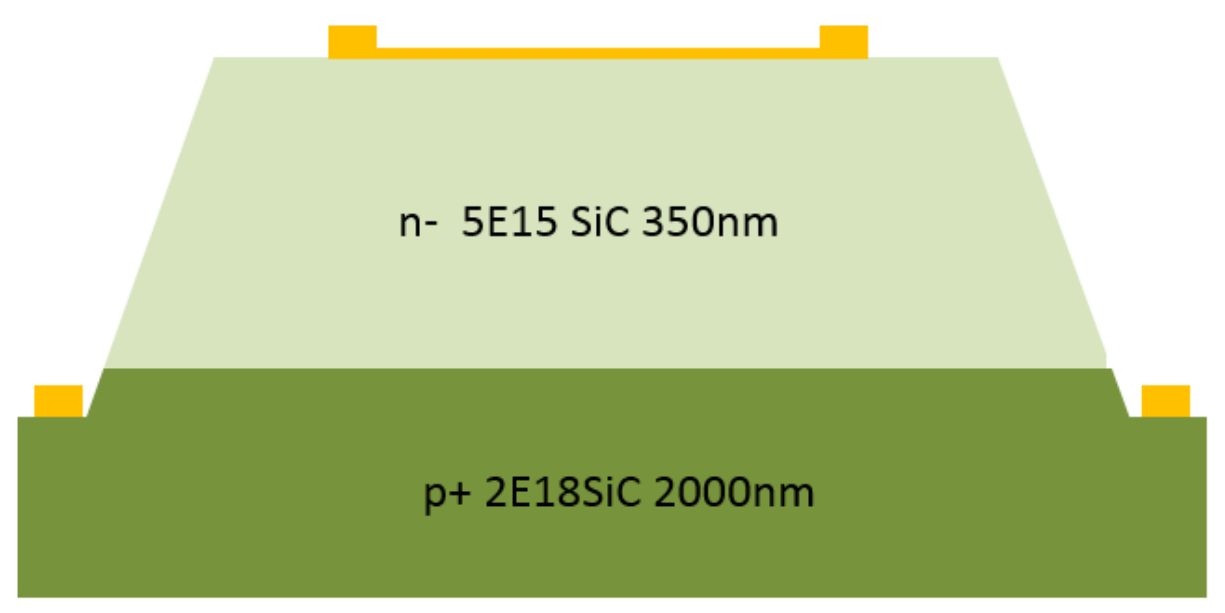

Figure 3-4. Device structure of the $\mathrm{SiC}$ metal-n-p photodiode

The SiC metal-n-p diode structure is shown in Figure 3-4. From bottom to top, it consists of a $2 \mu \mathrm{m}$-thick $\mathrm{p}+\mathrm{SiC}$ layer and a $350 \mathrm{~nm}$ n- SiC layer. A thin semi-transparent metal contact consisting of $\mathrm{Ni} 5 \mathrm{~nm} / \mathrm{Au} 5 \mathrm{~nm}$ was deposited in the central active region to ensure that the electric field is uniformly distributed across the active area and to make it semitransparent in the $200 \mathrm{~nm}$ to $400 \mathrm{~nm}$ spectral range. A semitransparent metal with the same thickness was also deposited on a sapphire wafer to measure the transmission spectrum. The measurement was done with the Perkin Elmer Lambda 950 spectrometer. The measured transmission of the semitransparent contact is shown in Figure 3-5. For comparison, a conventional $\mathrm{p}-\mathrm{n}-\mathrm{n}+\mathrm{SiC}$ photodetector was fabricated consisting of a $2-\mu \mathrm{m}$ p+ SiC layer, a $480 \mathrm{~nm} \mathrm{n-SiC} \mathrm{layer,} \mathrm{and} \mathrm{a} 100 \mathrm{~nm} n+$ SiC layer. 


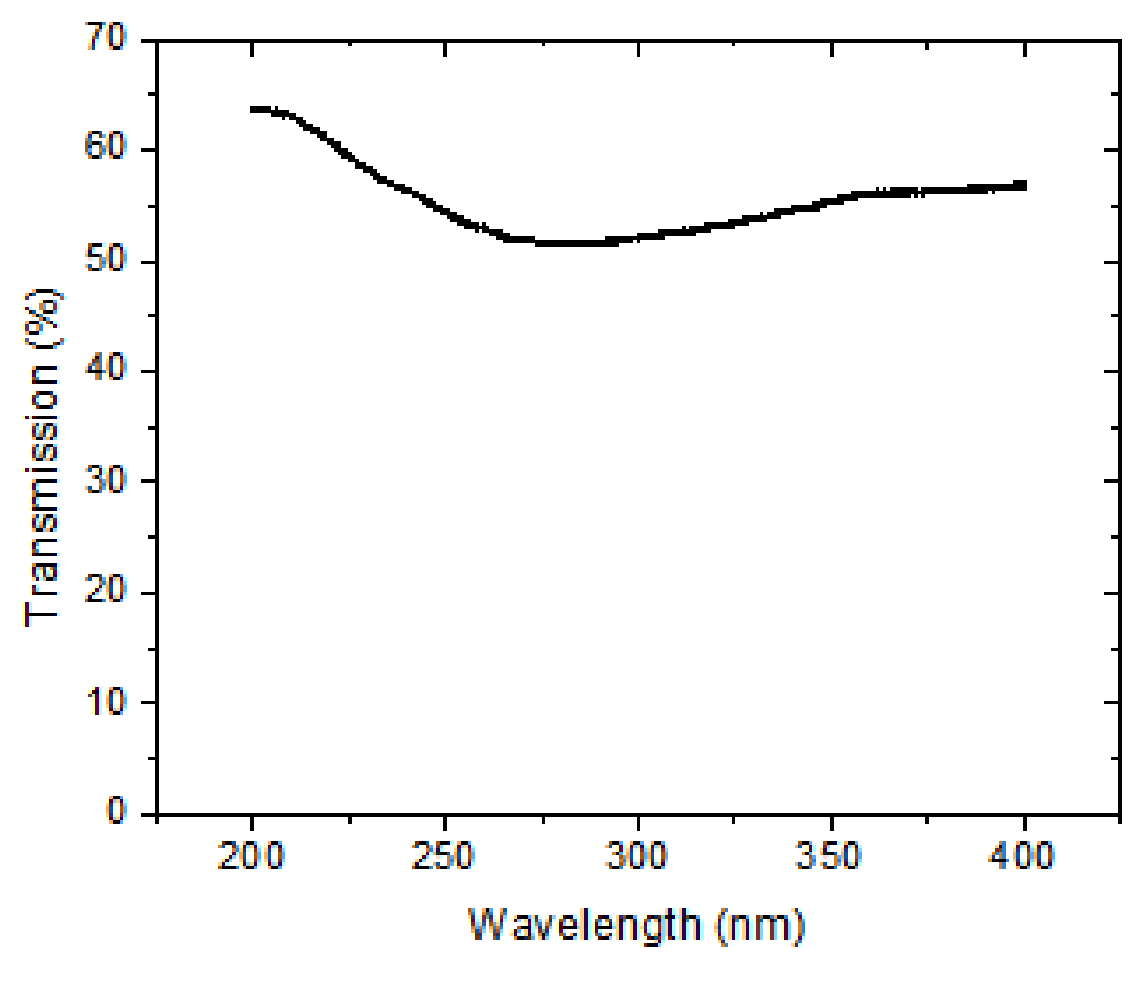

Figure 3-5. Transmission of the semitransparent contact

The current-voltage characteristic of a typical $50 \mu$ m-diameter metal-n-p photodiode is shown in Figure 3-6. Dark current less than $0.02 \mathrm{nA}$ is observed for reverse bias voltage less than $60 \mathrm{~V}$. Gain of 50 is estimated at reverse bias of $90 \mathrm{~V}$.

The measured external quantum efficiency at unity gain of typical metal-n- $-\mathrm{p}$ and $\mathrm{p}-\mathrm{n}-$ $\mathrm{n}+$ diodes are shown in Figure 3-7. The response of the p-n-n+ photodiode peaks at 270 $\mathrm{nm}$ and sharply decreases at shorter wavelength. In contrast, the response of the metal- $\mathrm{n}^{-}-$ p photodiodes is nearly flat from $270 \mathrm{~nm}$ to $225 \mathrm{~nm}$ with a slight decrease below $225 \mathrm{~nm}$ and a peak external quantum efficiency of $\sim 45 \%$. This response is greater than that observed for the p-n-n+ diode for wavelength shorter than $240 \mathrm{~nm}$ despite losses due to the 
semi-transparent metal contact. After accounting for these losses, the internal quantum efficiency at $255 \mathrm{~nm}$ is $81 \%$, which is significantly higher than that for $\mathrm{p}-\mathrm{n}-\mathrm{n}+$ devices.

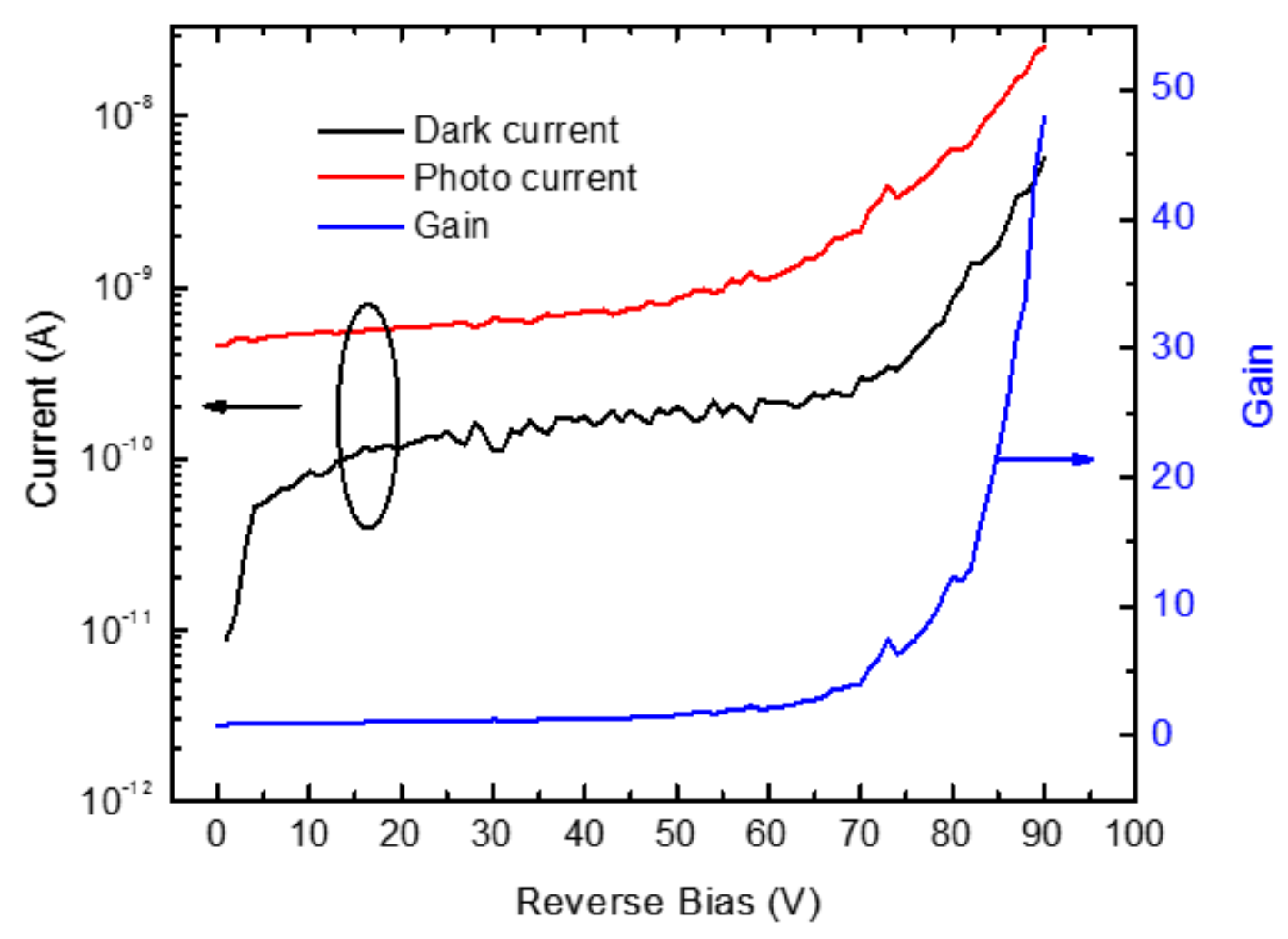

Figure 3-2. I-V of SiC metal-n־-p photodiode. 


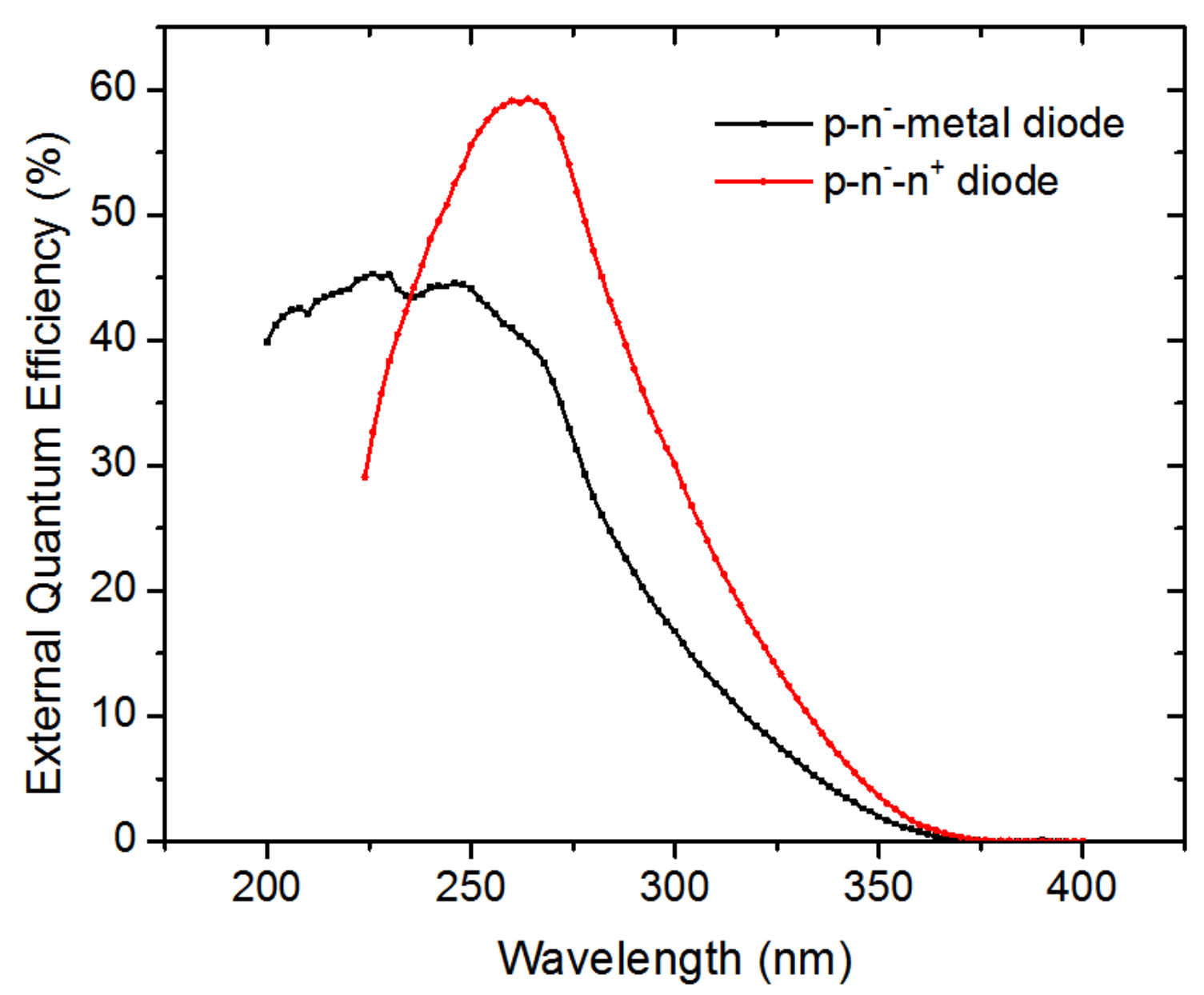

Figure 3-7. QE of metal-n־-p photodiode compared with n+-n־-p photodiode.

The enhancement of photo-response at short wavelength is attributed to improved collection of photo-generated carriers through drift within the high electric field region near the surface of the lightly-doped $\mathrm{SiC}$ absorption region despite the presence of surface states, while the observed decrease in photo-response at wavelengths shorter than $255 \mathrm{~nm}$ indicates that surface recombination still plays an important role. 


\subsection{SiC NIP photodiode with graded doping $\mathbf{n}$ layer}

As noted above, modeling of the spectral response of the SiC PIN APD shows that the high surface recombination velocity is responsible for the poor deep-UV response. Most of the carriers generated near the surface will move toward the surface and recombine through the states in the forbidden band. In order to stop the carriers from reaching the surface, a high-low junction utilizing a graded doping profile is incorporated into the highly-doped layer. Figure 3-8 shows the device structure design of the SiC NIP photodiode with graded doping in the top n-type region.

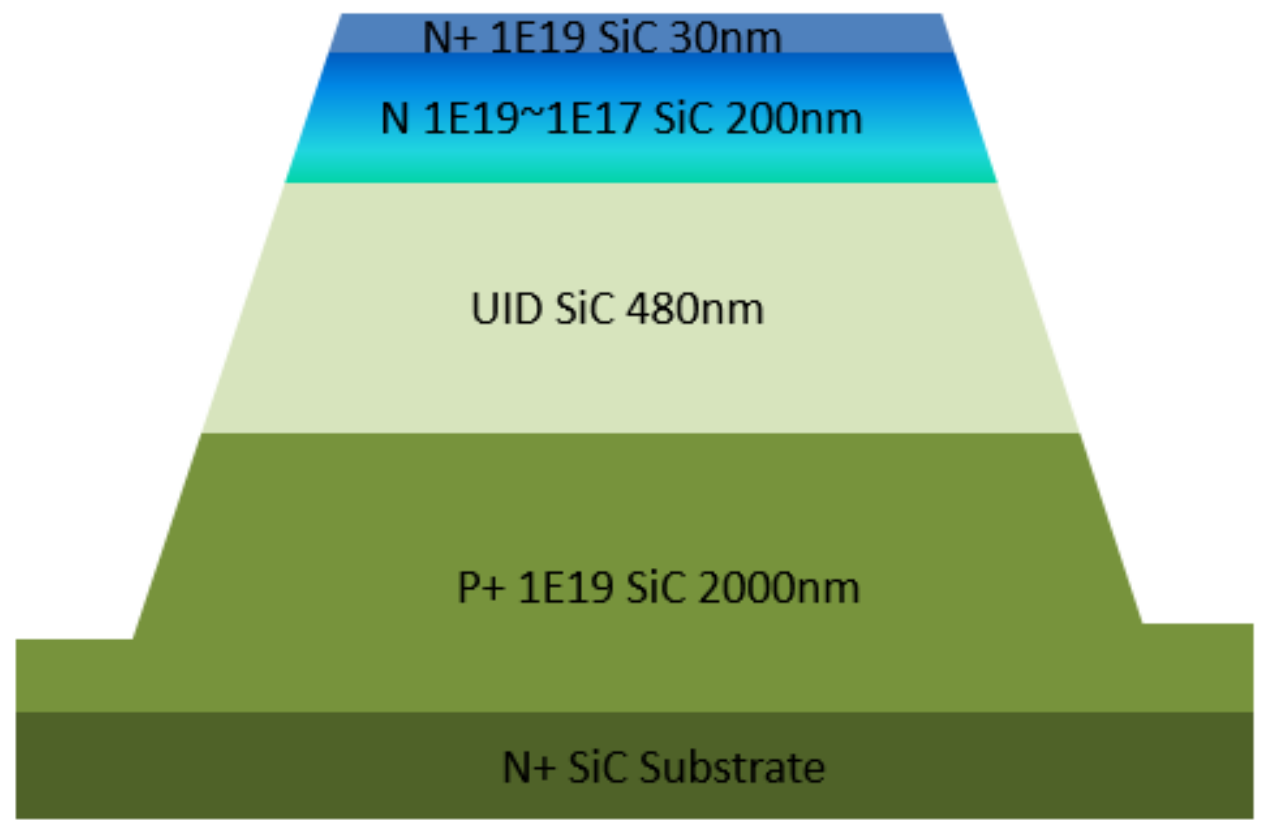

Figure 3-8. Cross section of the SiC NIP photodiode design

The wafer was grown by Etc-Epi Inc. From bottom to top, the structure I designed consists of a $\mathrm{SiC} \mathrm{n}+$ substrate with $2 \mu \mathrm{m}$ p+ layer, a $480 \mathrm{~nm}$ unintentionally doped (UID) 
layer, a $200 \mathrm{~nm}$ n layer with graded doping density changing from $1 \times 10^{17} \mathrm{~cm}^{-3}$ to $1 \times$ $10^{19} \mathrm{~cm}^{-3}$, and a $30 \mathrm{~nm} \mathrm{n}+$ top layer. The $30 \mathrm{~nm}$ top layer was added because approximately $30 \mathrm{~nm}$ of $\mathrm{SiC}$ will be consumed during the thermal oxidation step in the photodiode processing. We also sent a small piece of this wafer for Secondary Ion Mass Spectrometry (SIMS) analysis to verify the wafer doping profile and layer thickness. The SIMS result is shown in Figure 3-9. $\mathrm{Al}$ is the $\mathrm{p}$ type dopant for $\mathrm{SiC}$ and $\mathrm{N}$ is the $\mathrm{n}$ type dopant. The SIMS analysis shows that most of the layer thicknesses and the doping profile, including the graded doping, were well within the target range. However, the top layer thickness was measured to be $150 \mathrm{~nm}$ instead of the targeted $30 \mathrm{~nm}$.

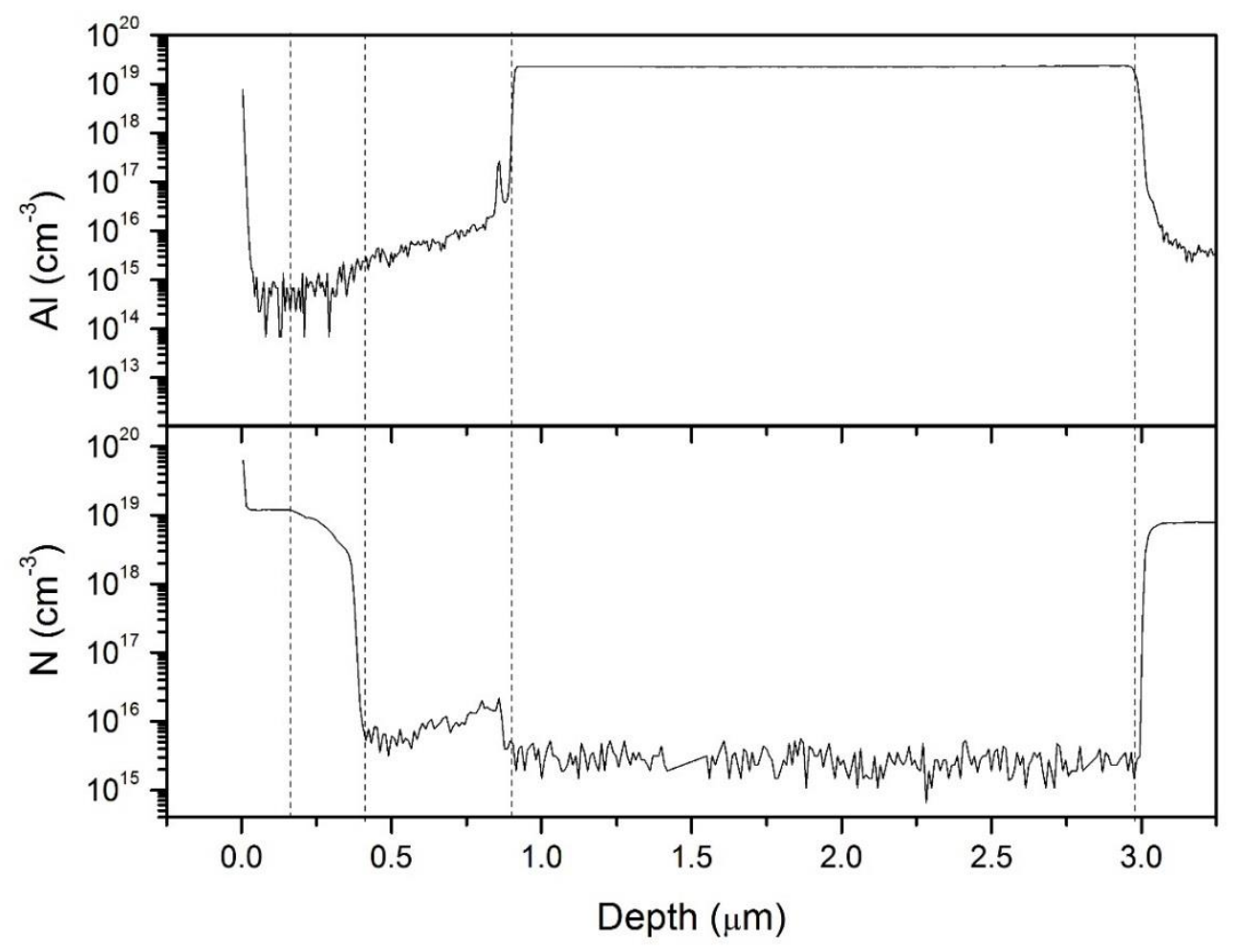

Figure 3-9. SIMS analysis of the SiC NIP wafer 
The wafer was fabricated into photodiodes using the process flow described in chapter 2. However, due to the thick top layer, a recessed window was added after mesa patterning; $120 \mathrm{~nm}$ of the top layer in the active area was removed by ICP etching. The etch depth was confirmed by the profile meter. Also the thickness of the $\mathrm{SiO}_{2}$ anti-reflection (AR) coating was optimized for $240 \mathrm{~nm}$.

The I-V characteristic of a $50 \mu$ m-diameter device is shown in Figure 3-10. The photodiode exhibited very low primary current near breakdown and high gain over 1000 was achieved. Dark current of $\sim 1 \mathrm{pA}$ was observed at gain of 100 .

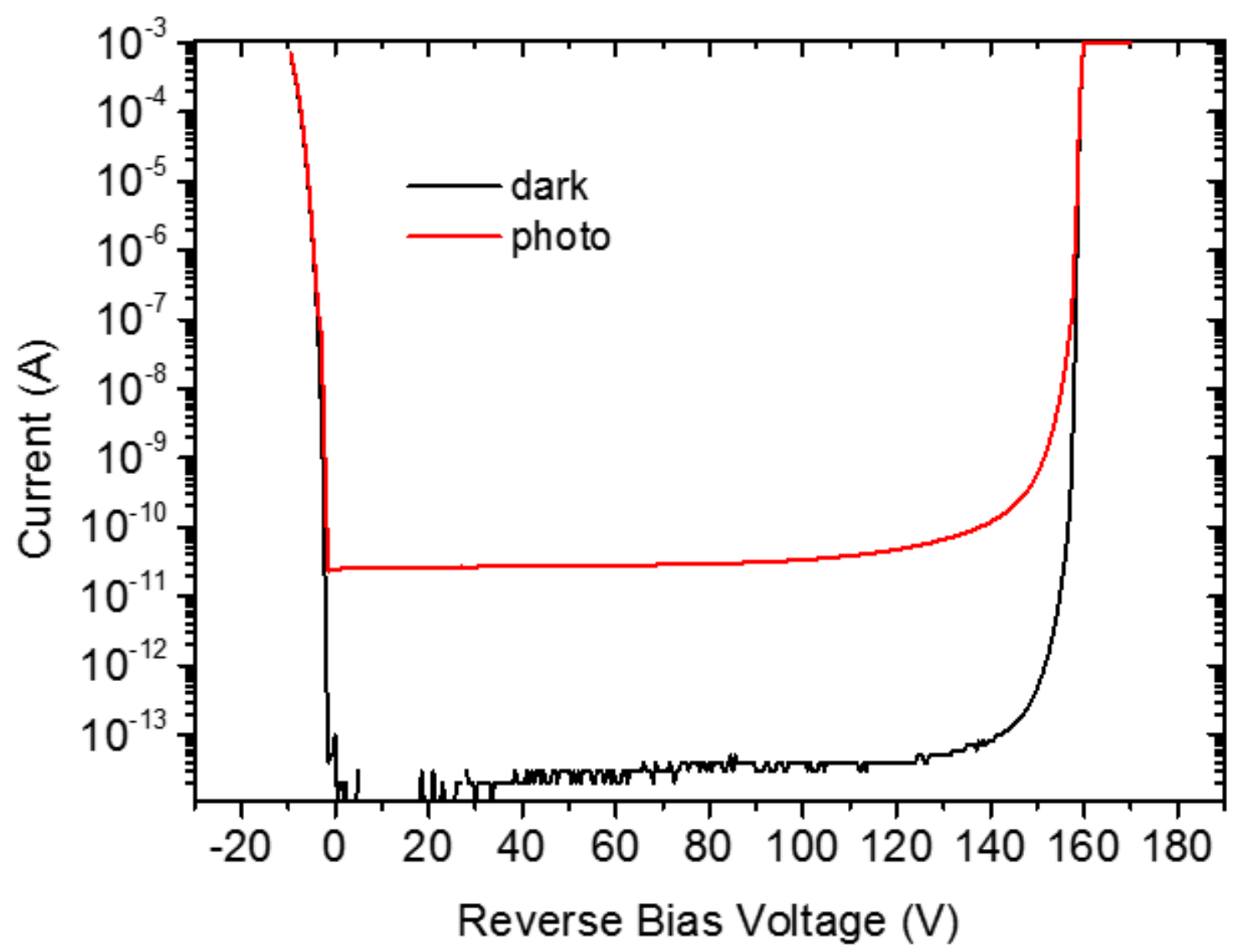

Figure 3-10. I-V characteristics of the SiC NIP photodiode 
External quantum efficiency measured at unity gain compared with a SiC PIN photodiode that was described earlier in this chapter with same i layer thickness is plotted in Figure 3-11. The measurement yielded peak quantum efficiency of $~ 55 \%$ at $270 \mathrm{~nm}$, which is about 7\% lower than the PIN structure. This might be due to the fact that the AR coating thickness was optimized at $240 \mathrm{~nm}$ instead of the peak quantum efficiency wavelength. It was also observed that for wavelength shorter than $240 \mathrm{~nm}$ the efficiency is enhanced, which is consistent with the motivation of this design. The quantum efficiency at $240 \mathrm{~nm}$ was improved from $\sim 1 \%$ to $\sim 30 \%$, which is believed to be a result of the builtin electric field in the $\mathrm{n}$ layer created by the graded doping profile.

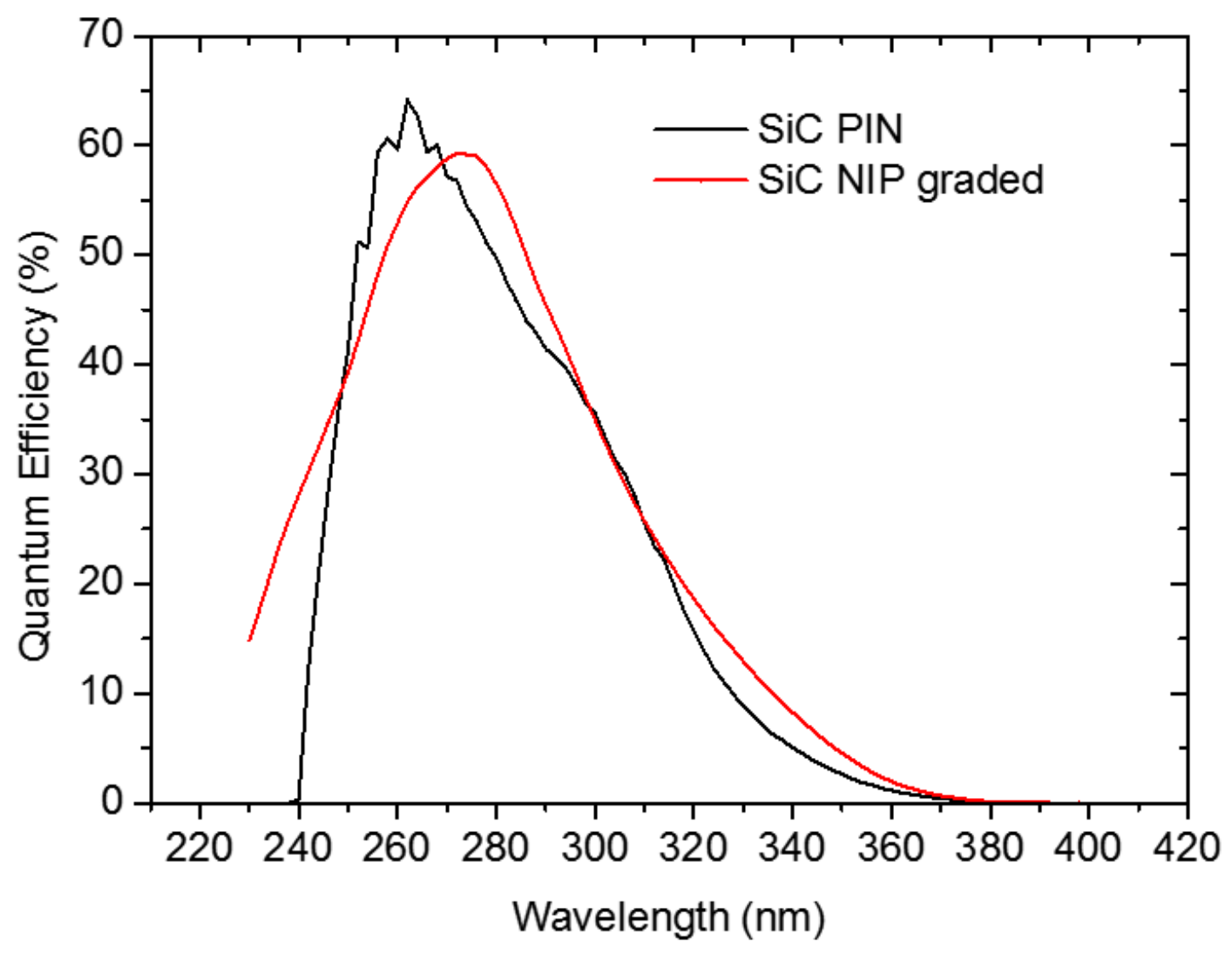

Figure 3-11. External quantum efficiency of the SiC NIP APD with graded $n$ layer 


\subsection{Conclusion}

In this chapter, the spectral response of the SiC APDs with PIN structure has been studied by external quantum efficiency modeling. The contributions from carriers generated in different layers are analyzed. The results show over $75 \%$ of the deep-UV photons are absorbed in the two highly-doped top layers, but less than half of these carriers diffuse into the high electric field region and get collected. This is due to the fact that the diffusion length in the highly-doped $\mathrm{SiC}$ is relatively short and to the high surface recombination velocity. The surface band bending, which is the physical explanation of the high surface recombination, is also discussed.

Different approaches to enhance the deep-UV response of the SiC APD are discussed and two of them are investigated in this chapter. The SiC metal- $\mathrm{n}^{-}-\mathrm{p}$ photodiode demonstrated flat external quantum efficiency of $\sim 45 \%$ from $270 \mathrm{~nm}$ to $225 \mathrm{~nm}$ with a slight decrease below $225 \mathrm{~nm}$. This response is greater than that observed for the p-n-n+ diode for wavelength shorter than $240 \mathrm{~nm}$ despite losses due to the semi-transparent metal contact. The deep-UV response enhancement results from the improved collection of photo-generated carriers through drift within the high electric field region near the surface of the lightly doped $\mathrm{SiC}$ absorption region despite the presence of surface states. However, these devices exhibited high dark current due to the high electric field at metal/SiC interface.

The SiC NIP photodiode with graded doping profile in the n layer utilizes a high-low junction to provide an effective electric field that can restricts carriers from reaching the top surface. An enhanced deep-UV response was observed with the external quantum 
efficiency of $\sim 30 \%$ at $240 \mathrm{~nm}$. Also the dark current at high bias voltage is as low as the SiC p-i-n APD. 


\section{Chapter 4. AlGaN/SiC Photodiode for Deep-UV detection}

In the previous chapter, I have investigated various approaches to enhance the deepUV response of homojunction SiC APDs. In this chapter, I will focus on the $\mathrm{AlGaN} / \mathrm{SiC}$ heterojunction structure with $\mathrm{AlGaN}$ heteroepitaxially grown on $\mathrm{SiC}$ epitaxial layers. The goal of this project is to extend the spectral response of SiC APD into shorter wavelength utilizing the optical properties of AlGaN. This work was also done in collaboration with researchers at the Army Research Laboratory in Adelphi, MD.

\subsection{AlGaN/SiC SAM avalanche photodiode}

As discussed in previous chapters, $\mathrm{SiC}$ APDs exhibit high avalanche gain, low dark current, and low excess noise, but suffer from poor deep-UV response due to a shallow absorption profile and surface recombination. Conversely, aluminum gallium nitride $(\mathrm{AlGaN})$ alloys have a direct bandgap that can be engineered for efficient absorption from $3.4 \mathrm{eV}$ to $6.2 \mathrm{eV}$, but they exhibit large leakage current [4.1]. Furthermore, AlGaN has a k-factor, a figure of merit for excess noise in APDs, close to 1, a signature of high excess noise [4.2]. To combine the advantages of $\mathrm{SiC}$ and $\mathrm{AlGaN}$, a separate absorption and multiplication (SAM) APD has been designed using AlGaN as the absorption layer and $\mathrm{SiC}$ as the multiplication layer. The SAM structure allows the peak response to be tuned below $260 \mathrm{~nm}$ by adjusting the AlN mode fraction in the AlGaN layer. AlGaN/SiC SAM APDs differ from traditional APDs in several important respects, including: (1) The AlGaN 
absorption region has a wider bandgap than the $\mathrm{SiC}$ multiplication region and therefore the photo-response will include contributions from absorption in both layers. (2) The AlGaN is grown on lattice mismatched $\mathrm{SiC}$, which introduces a high density of defects in the $\mathrm{AlGaN}$ [4.3]. As a result, the carrier diffusion length is short and an electric field in the AlGaN absorption region is required for carrier collection. (3) Both $\mathrm{SiC}$ and $\mathrm{AlGaN}$ are polar materials and the smaller spontaneous polarization of $\mathrm{SiC}$ as compared to $\mathrm{AlGaN}$ results in the formation of significant positive polarization induced charge at the interface between $\mathrm{SiC}$ and $\mathrm{AlGaN}$ [4.4]. This interfacial charge will confine the electric field in the SiC layer.

One way to extend the electric field into the AlGaN absorption layer is to insert an $\mathrm{AlN}$ layer, which has larger spontaneous polarization than both the $\mathrm{AlGaN}$ absorption and $\mathrm{SiC}$ multiplication regions [4.5]. This layer creates a two-dimensional electron gas (2DEG) in the $\mathrm{SiC}$ multiplication region and two-dimensional hole gas in the $\mathrm{AlGaN}$ absorption region, with the effect of reducing the interface charge density. However, the AlN layer also forms a barrier for hole injection from $\mathrm{AlGaN}$ to $\mathrm{SiC}$ and electron injection from $\mathrm{SiC}$ to $\mathrm{AlGaN}$. Under reverse bias, the height of the barrier is significantly reduced. It is expected that the carriers will tunnel though the reduced barrier if the AlN layer is thin enough. Figure 4-1 shows a simulation of the band diagrams of the SiC/AlGaN heterojunction with and without an AlN layer. 

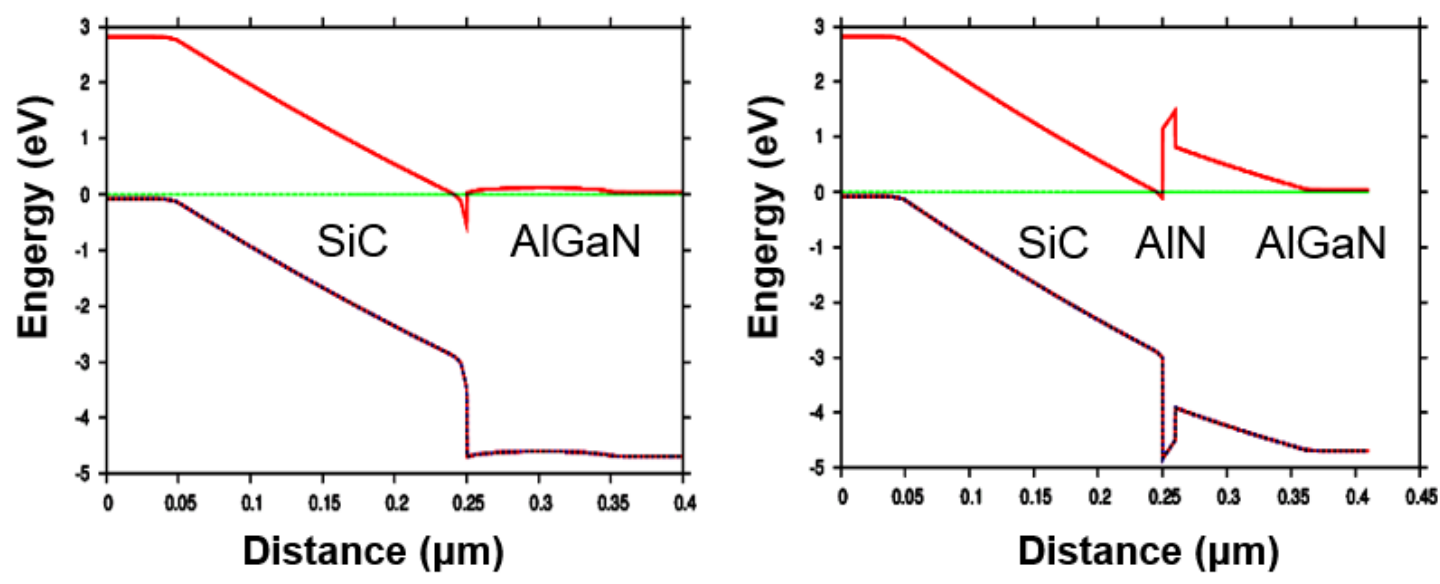

Figure 4-1. Comparison of a) thick i-layer PIN and b) SACM structure.

The influence of the AlN layer on the interface charge and device behavior is studied by comparing the photo response of an $\mathrm{Al}_{0.62} \mathrm{Ga}_{0.38} \mathrm{~N} / \mathrm{SiC}$ SAM APD with and without AlN layers. The structure of the $\mathrm{Al}_{0.62} \mathrm{Ga}_{0.38} \mathrm{~N} / \mathrm{SiC}$ APD is shown in Figure 4-2. From top to bottom, it consists of a $50 \mathrm{~nm}$ n-doped $\mathrm{Al}_{0.62} \mathrm{Ga}_{0.38} \mathrm{~N}$ contact layer, a $300 \mathrm{~nm}$ lightly $\mathrm{n}$ doped $\mathrm{Al}_{0.62} \mathrm{Ga} 0.38 \mathrm{~N}$ absorption layer, a $470 \mathrm{~nm}$ lightly $\mathrm{n}$-doped $\mathrm{SiC}$ multiplication layer, and a $2000 \mathrm{~nm}$ p-doped $\mathrm{SiC}$ buffer layer. The $\mathrm{SiC}$ substrate with two epitaxial layers was purchased from Cree, Inc. The $\mathrm{Al}_{0.62} \mathrm{Ga}_{0.38} \mathrm{~N}$ epitaxial layer was grown by Molecular Beam Epitaxy (MBE) by our collaborators at the Army Research Laboratory. The AlGaN/SiC photodiodes were fabricated using the process flow described in chapter 2 . 


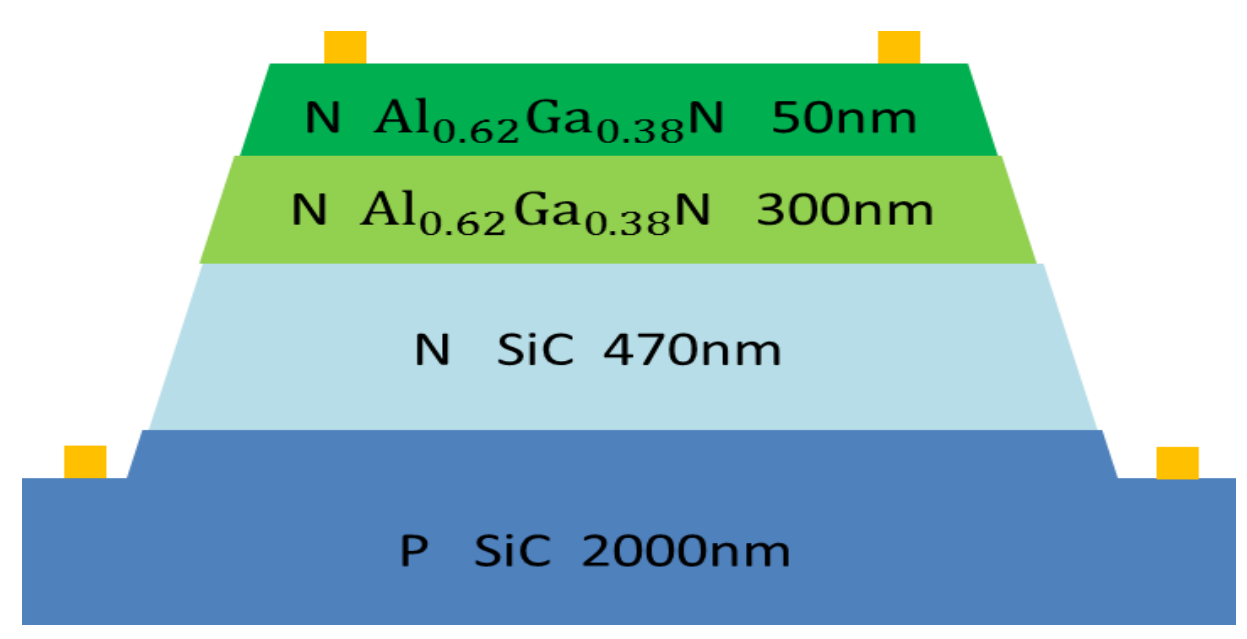

Figure 4-2. Cross section of the AIGaN/SiC SAM APD

Figure 4-3 shows the external quantum efficiency of the devices with and without the AlN layer. The photo response of these devices is expected to consist of two components, one arising from absorption in the $\mathrm{Al}_{0.62} \mathrm{Ga}_{0.38} \mathrm{~N}$ region and the other arising from the $\mathrm{SiC}$ multiplication region. The bandgap of $\mathrm{Al}_{0.62} \mathrm{Ga}_{0.38} \mathrm{~N}$ is $4.84 \mathrm{eV}$, which results in a cutoff wavelength at $255 \mathrm{~nm}$. Most of the light with wavelength shorter than $255 \mathrm{~nm}$ is absorbed in the $\mathrm{Al}_{0.62} \mathrm{Ga}_{0.38} \mathrm{~N}$ layer.

From Figure 4-3a, the response of the device without AlN layer looks similar to homojunction SiC APDs. The shape of the curve did not change with bias voltage. The sharp cut-off near $250 \mathrm{~nm}$ indicates that the photo-generated carriers in the $\mathrm{Al}_{0.62} \mathrm{Ga}_{0.38} \mathrm{~N}$ absorption layer are not collected due to the short diffusion length. The $\mathrm{Al}_{0.62} \mathrm{Ga}_{0.38} \mathrm{~N}$ layer principally acts as long pass optical filter. 

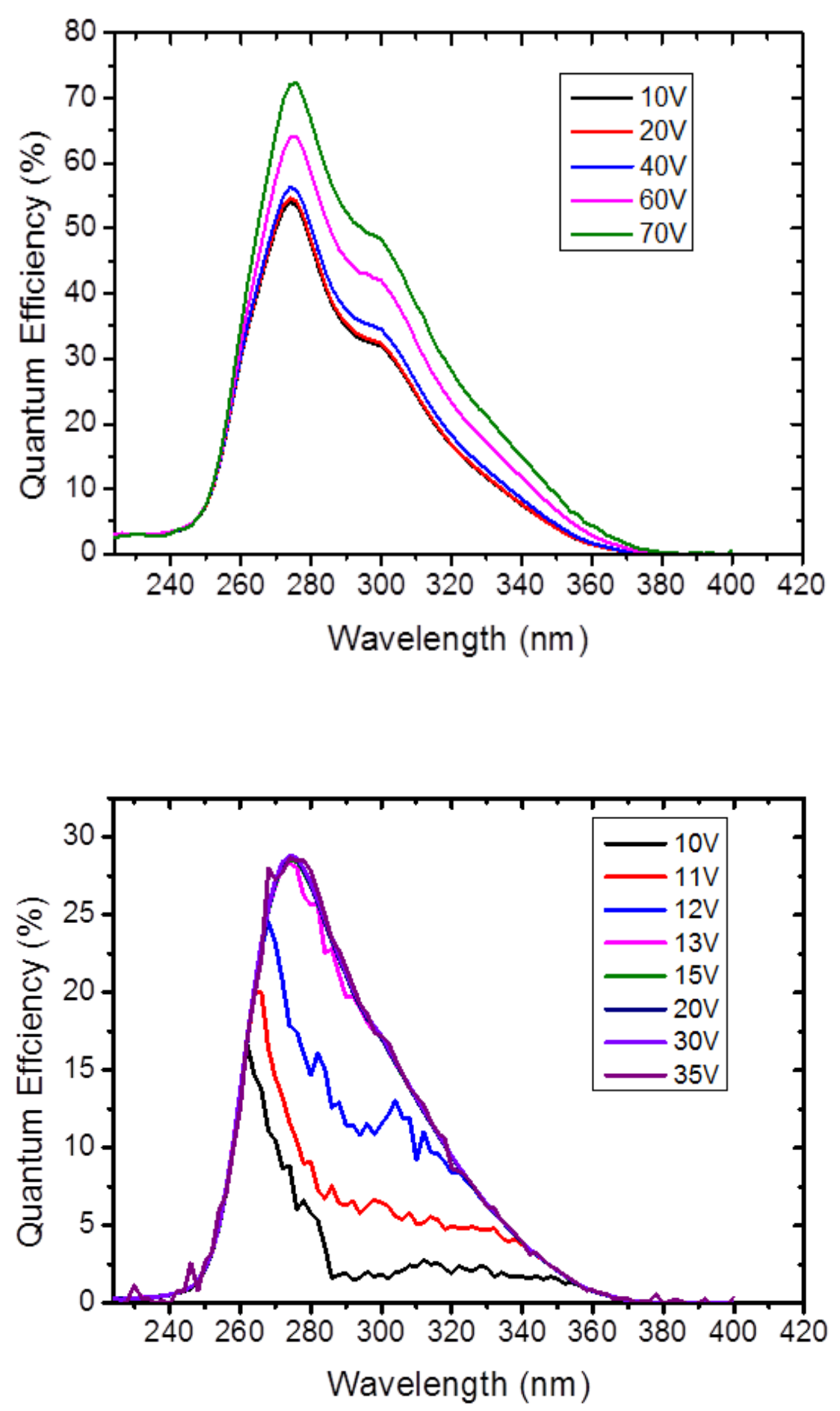

Figure 4-3. External quantum efficiency of AIGaN/SiC SAM APD without (a) and with AIN layer (b) [ You need to label the figures (a) and (b).] 
In contrast, the response of the device with $10 \mathrm{~nm}$ AlN layer shown in Figure 4-3b has a bias-dependent behavior. This is an indication that here is a barrier at the interface. The increase in long-wavelength response results from reduction in the barrier height that is associated with the AlN layer at the interface. As the applied reverse bias is increased, the barrier is lowered and electrons generated from lower energy photons in the $\mathrm{SiC}$ are able to surmount it and contribute to the photo-response. We note that the curve also drops sharply at $250 \mathrm{~nm}$, which means that the carriers generated in the $\mathrm{Al}_{0.62} \mathrm{Ga}_{0.38} \mathrm{~N}$ layer are still lost. This is due to the fact that the photo-generated holes in the $\mathrm{Al}_{0.62} \mathrm{Ga}_{0.38} \mathrm{~N}$ do not have enough energy to tunnel through the $10 \mathrm{~nm}$ AlN layer to SiC. The lower peak quantum efficiency can also be attributed to recombination through defects at SiC/AlN interface.

Overall, the AlN layer is effective in reducing the polarization-induced interface charge, but optimization of the thickness is required for higher injection efficiency of carriers generated in the $\mathrm{Al}_{0.62} \mathrm{Ga}_{0.38} \mathrm{~N}$ absorption region.

\subsection{SiC avalanche photodiode with AIGaN window layer}

An alternative approach to increase the absorption of the deep-UV photons in the highelectric-field, lightly-doped $\mathrm{SiC}$ absorption region while reducing absorption losses in the deep UV is to employ an $\mathrm{Al}_{0.8} \mathrm{Ga}_{0.2} \mathrm{~N}$ n-type doped contact window layer that is transparent to light with wavelength longer than $230 \mathrm{~nm}$. Figure $4-4$ shows a cross section of the $\mathrm{Al}_{0.8} \mathrm{Ga}_{0.2} \mathrm{~N} / \mathrm{AlN} / \mathrm{SiC}$ device. 


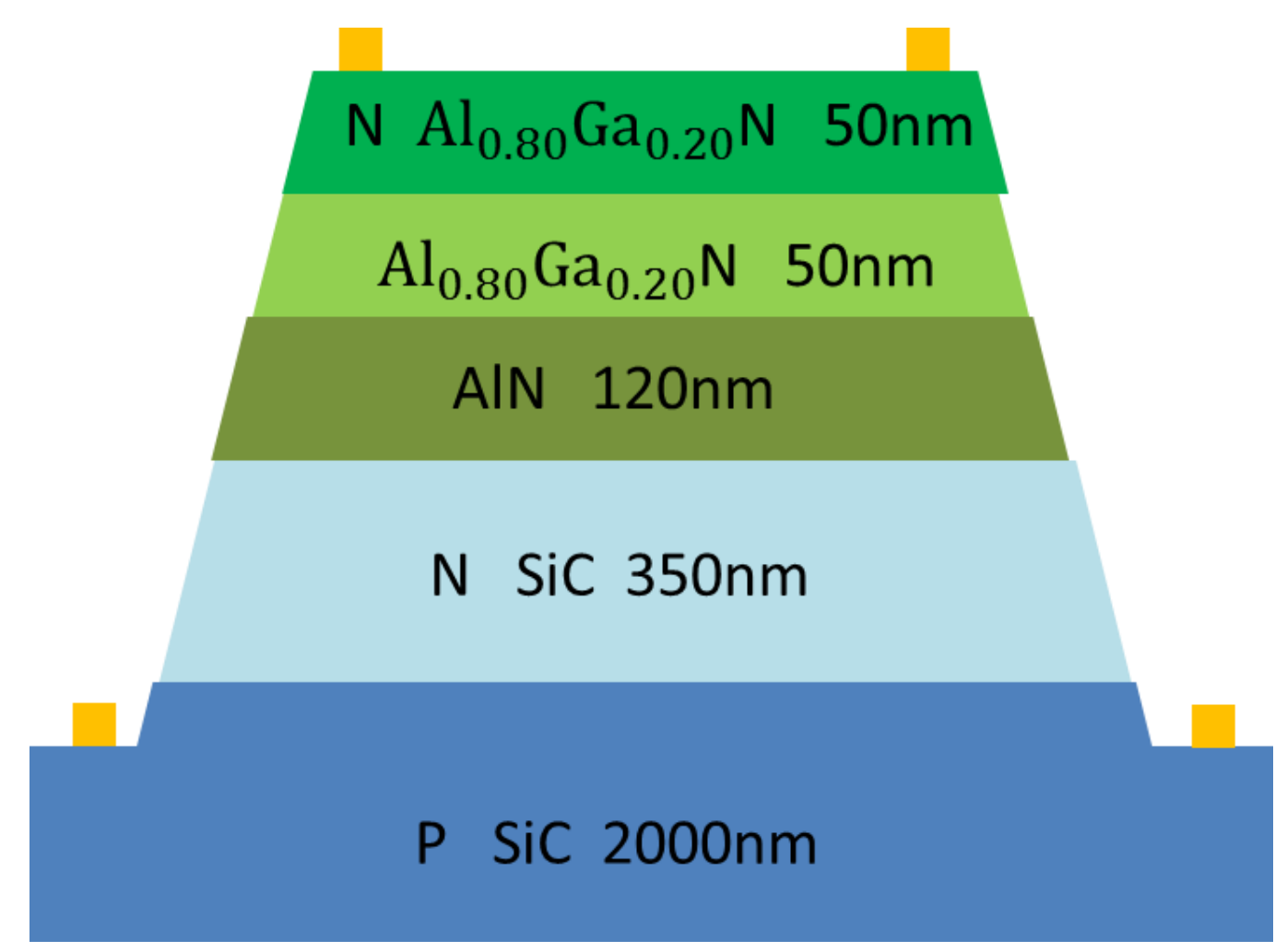

Figure 4-4. Cross section of $\mathrm{Al}_{0.8} \mathrm{Ga}_{0.2} \mathrm{~N} / \mathrm{AIN} / \mathrm{SiC}$ photodiode

The external quantum efficiency versus bias voltage is shown in Figure 4-5. The spectral response of this detector shows a strong cut-off at short wavelength and a peak that shifts from $230 \mathrm{~nm}$ to $242 \mathrm{~nm}$ when the bias voltage is increase from $10 \mathrm{~V}$ to $40 \mathrm{~V}$. A peak external quantum efficiency of $\sim 80 \%$ is observed at $242 \mathrm{~nm}$, which is significantly higher than the conventional homojunction PIN SiC detector. This can be explained by the shift of the absorption in $\mathrm{Al}_{0.8} \mathrm{Ga}_{0.2} \mathrm{~N}$. The band gap of $\mathrm{Al}_{0.8} \mathrm{Ga}_{0.2} \mathrm{~N}$ is $5.38 \mathrm{eV}$ [4.6], which corresponds to an absorption edge at approximately $230 \mathrm{~nm}$. So this enhancement results from increased absorption of the deep-UV photons in the depleted SiC layer. Carriers generated in this spectral range are then collected more efficiently by drift in this region. 


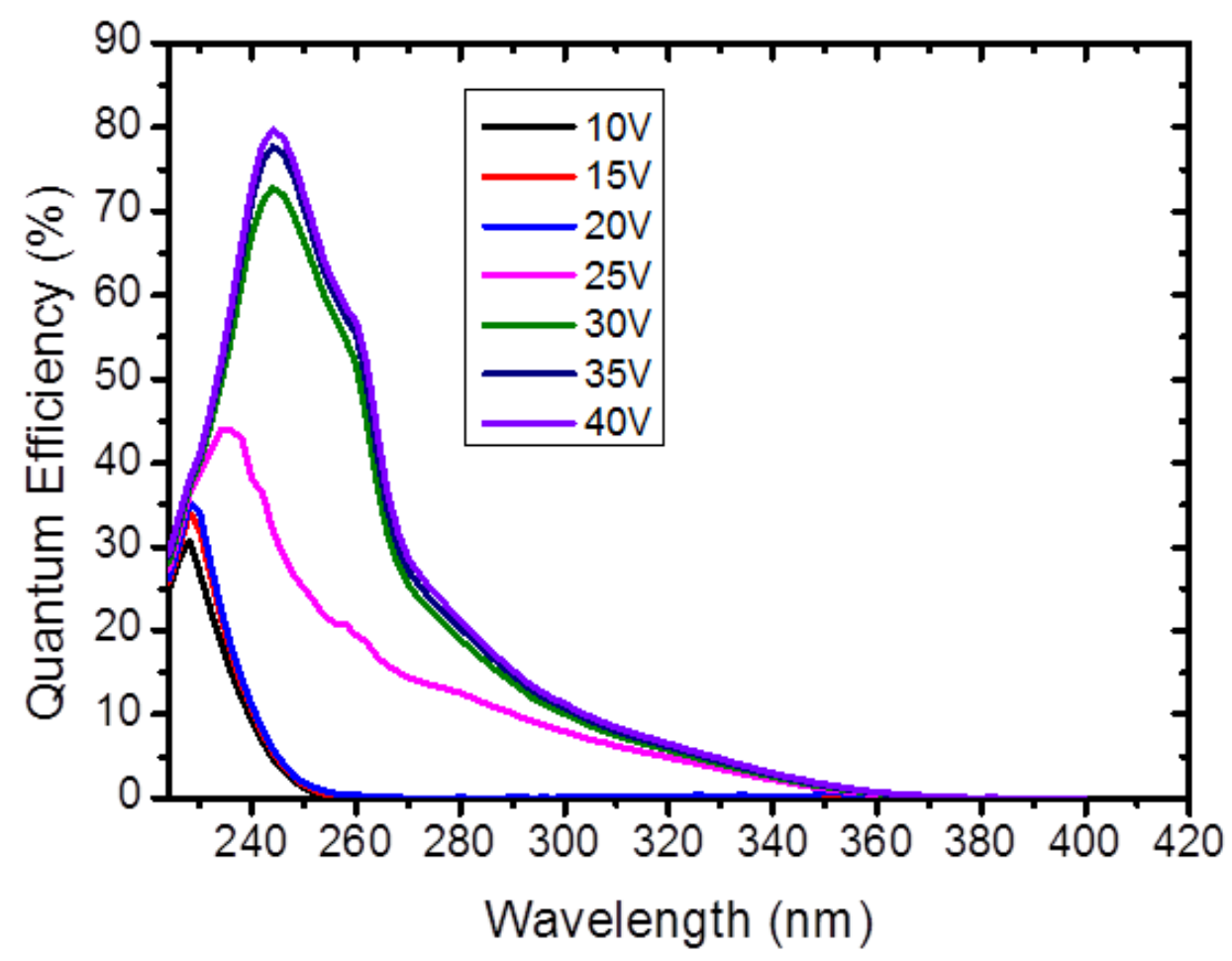

Figure 4-5. External quantum efficiency of $\mathrm{Al}_{0.8} \mathrm{Ga}_{0.2} \mathrm{~N} / \mathrm{AIN} / \mathrm{SiC}$ photodiode

The increase and red-shift of the photo-response with increasing reverse bias and the sharp cutoff at $242 \mathrm{~nm}$ is explained by the presence of a barrier associated with polarization-induced charge at the hetero-interfaces as shown if Figure 4-6. The Al-polar AlN grown on $\mathrm{SiC}$ give rise to positive polarization-induced charge at the $\mathrm{AlN} / \mathrm{SiC}$ interface. The $\mathrm{Al}_{0.8} \mathrm{Ga}_{0.2} \mathrm{~N}$ grown on $\mathrm{AlN}$ results in genitive polarization-induced charge at the $\mathrm{Al}_{0.8} \mathrm{Ga}_{0.2} \mathrm{~N} / \mathrm{AlN}$ interface. Considering the thickness of the AlN barrier layer, this barrier is significantly larger than the band offsets between these materials and prevents holes generated within the $\mathrm{Al}_{0.8} \mathrm{Gaa}_{0.2} \mathrm{~N}$ window layer from being collected regardless of reverse bias. As a result, this layers acts as an optical filter that cuts off the short- 
wavelength response of the photodiode above the band gap energy of the $\mathrm{Al}_{0.8} \mathrm{Ga}_{0.2} \mathrm{~N}$ window layer.

The relative lack of bias dependence on the response at wavelength shorter than 224 $\mathrm{nm}$ indicates that this barrier has minimal impact on electrons that are generated in higher of $4 \mathrm{H}-\mathrm{SiC}$ [4.7], as these carriers can have sufficient energy and the relaxation time allows them to traverse the barrier. Increasing the reverse bias reduces the barrier, resulting in a red-shift in photo-response associated with more electrons that are excited to lower bands having sufficient energy to cross it. The strong suppression of the photo response at wavelength longer than $260 \mathrm{~nm}$ for all reverse bias indicates that the barrier is still sufficient at the highest bias investigated to reduce the collection of the associated photoexcited carriers in the bottom of $\mathrm{M}$ and lower L valleys.

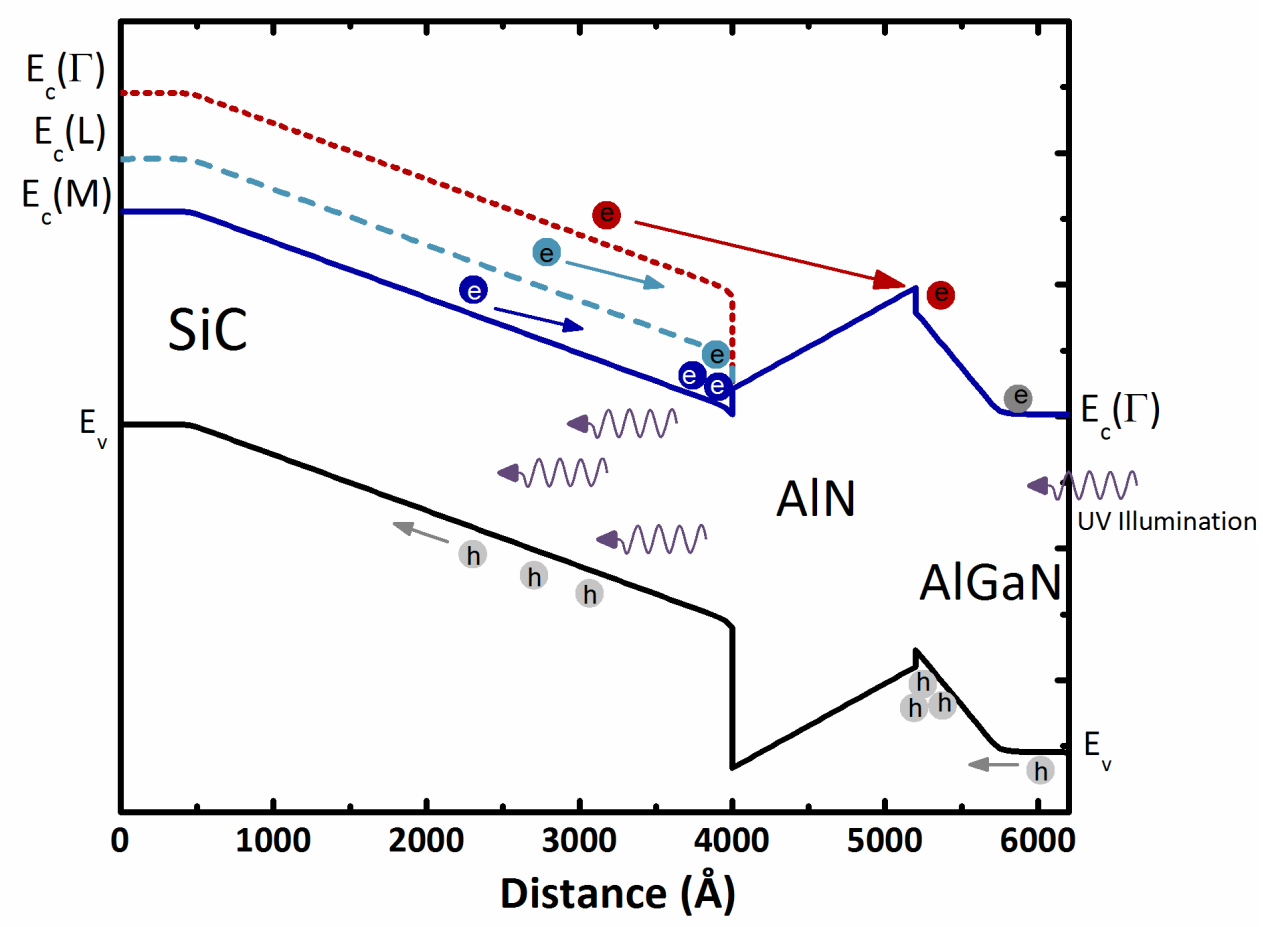

Figure 4-6. Band diagram of $\mathrm{SiC} / \mathrm{AlN} / \mathrm{Al}_{0.8} \mathrm{Ga}_{0.2} \mathrm{~N}$ for different band in $\mathrm{SiC}$ 
Given the fact that the $\mathrm{Al}_{0.8} \mathrm{Ga}_{0.2} \mathrm{~N}$ window layer has proved to be effective in enhancing the deep-UV response of the SiC APD, a modified structure was designed to further improve the performance. A cross section of that structure is shown in Figure 4-7. The material of the window layer was changed from $\mathrm{Al}_{0.8} \mathrm{Ga}_{0.2} \mathrm{~N}$ to $\mathrm{Al}_{0.9} \mathrm{Ga}_{0.1} \mathrm{~N}$. The motivation is to increase the bandgap energy of the window layer by increasing the Al mole fraction.. As a result, more photons with higher energy are able to pass through the window layer and get absorbed in the $\mathrm{SiC}$ n- layer, with a projected blue shift of the spectral response. Also the AlN layer between the $\mathrm{AlGaN}$ and $\mathrm{SiC}$ layer was removed so that there will be no barriers for the electrons that in the lower $\mathrm{L}$ and $\mathrm{M}$ valleys. The suppression of the photo response at longer wavelength discussed in the structure above is expected to be greatly reduced.

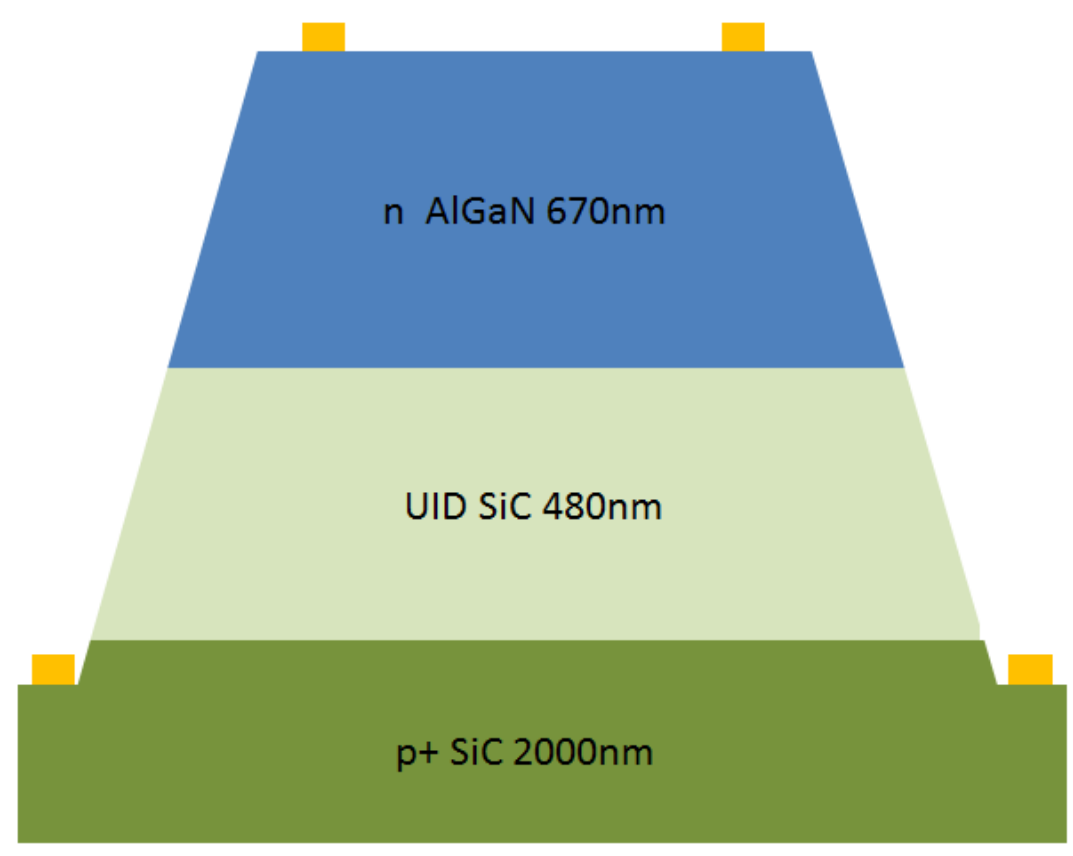

Figure 4-7. Cross section of $\mathrm{Al}_{0.9} \mathrm{Ga}_{0.1} \mathrm{~N} / \mathrm{SiC}$ photodiode 
The photoresponse of the $\mathrm{Al}_{0.9} \mathrm{Ga}_{0.1} \mathrm{~N} / \mathrm{SiC}$ photodiode and homojunction $\mathrm{SiC}$ photodiodes are shown in Figure 4-8. The recessed window SiC photodiode has a peak quantum efficiency of $\sim 60 \%$ at $260 \mathrm{~nm}$ and a sharp drop off in response at shorter wavelength. In contrast, the $\mathrm{Al}_{0.9} \mathrm{Ga}_{0.1} \mathrm{~N} / \mathrm{SiC}$ photodiode has a flatter response in the deepUV that exceeds $60 \%$ over a spectral range from $215-256 \mathrm{~nm}$ and a sharp cut-off at shorter wavelengths. This enhanced deep-UV response is attributed to the generation of carriers within the depletion region of the heterojunction, where these carriers are more efficiently collected through drift as shown in Figure 4-9. Importantly, the presence of fixed, positive, polarization-induced charge at the hereto-interface arising from the larger spontaneous polarization of $\mathrm{AlGaN}$ over that of $\mathrm{SiC}$ should act to repel holes from the interface so as to further suppress interface recombination. However, additional studies are required to understand the relative contributions of each of these effects. The sharp short-wavelength cutoff observed at $210 \mathrm{~nm}$ is attributed to the absorption of photons in the $\mathrm{Al}_{0.9} \mathrm{Ga}_{0.1} \mathrm{~N}$ window layer. The short carrier diffusion lengths within this region associated with the presence of dislocations arising from lattice mismatch inhibits the collection of photogenerated carriers through diffusion. 


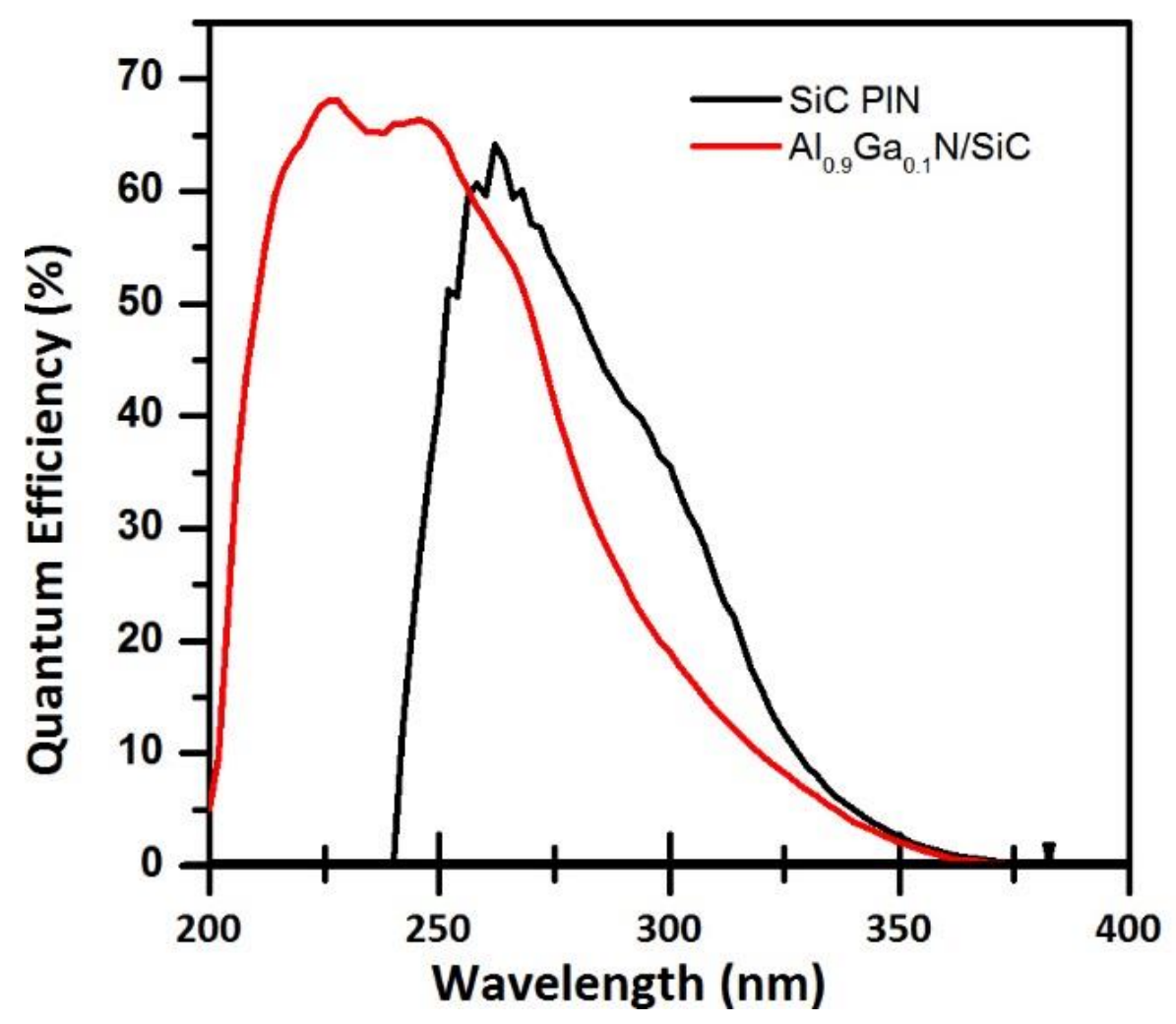

Figure 4-8. Quantum efficiency of $\mathrm{Al}_{0.9} \mathrm{Ga}_{0.1} \mathrm{~N} / \mathrm{SiC}$ and $\mathrm{SiC} \mathrm{PIN}$ photodiode
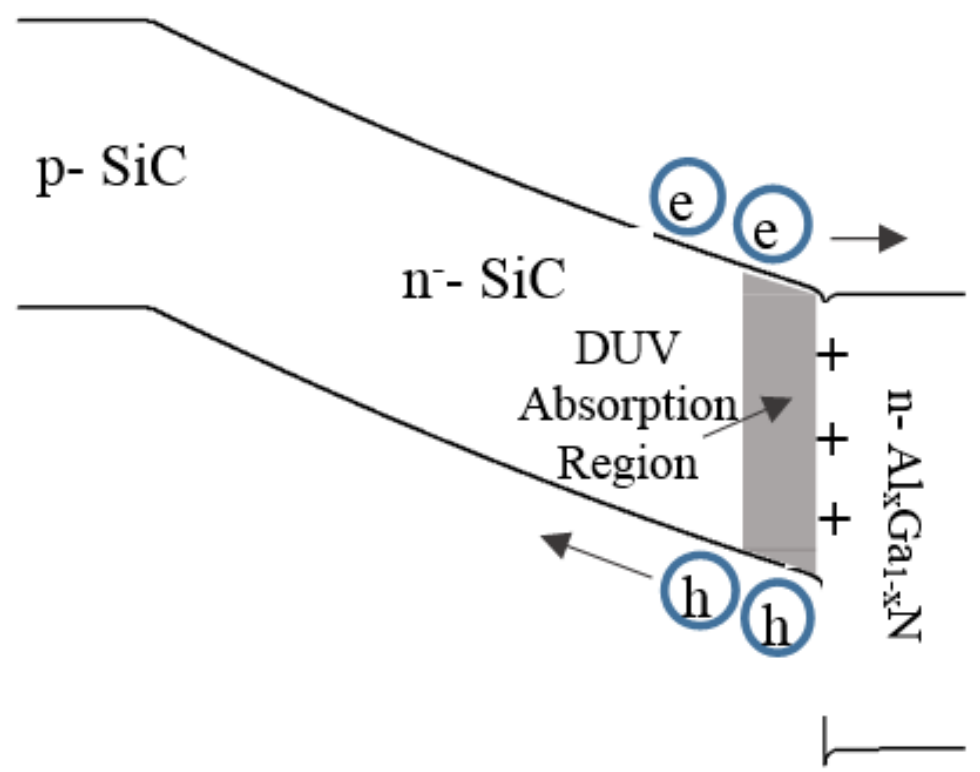

Figure 4-9. Band diagram of $\mathrm{Al}_{0.9} \mathrm{Ga}_{0.1} \mathrm{~N} / \mathrm{SiC}$ photodiode 


\subsection{Conclusion}

$\mathrm{AlGaN} / \mathrm{SiC}$ heterogeneous photodiodes were investigated for deep-UV detection in this chapter. Both reach-through and non-reach-through structure were studied.

The $\mathrm{AlGaN} / \mathrm{SiC}$ SAM structure is investigated as the early effort to extend the spectral response of SiC into deep-UV. However, the large polarization induced charge formed at the hetero-interface is detrimental to device performance, because it confines the electric field to the $\mathrm{SiC}$ multiplication layer. An AlGaN/AlN/SiC SAM structure with $\mathrm{Al}_{0.62} \mathrm{Ga}_{0.38} \mathrm{~N}$ as absorption layer was fabricated and characterized. The AlN layer proved to be effective in reducing the polarization-induced charge and extending the electric field into the $\mathrm{AlGaN}$. However the bias dependent behavior of the AlGaN/AlN/SiC indicates that the AlN acts as a barrier layer, thus a further optimization of the AlN layer thickness is needed.

The $\mathrm{SiC}$ with $\mathrm{AlGaN}$ transparent window layer provides another way to achieve high deep-UV response by absorbing the deep-UV photons in the depleted SiC layer. The $\mathrm{Al}_{0.8} \mathrm{Ga}_{0.2} \mathrm{~N} / \mathrm{AlN} / \mathrm{SiC}$ photodiodes exhibited a peak quantum efficiency of $\sim 80 \%$ at $242 \mathrm{~nm}$. However, the AlN layer formed a barrier between the AlGaN and SiC, especially for carriers with lower energy in the SiC L and M valleys. In order to remove the barrier effect and further enhance the deep-UV response, photodiodes an $\mathrm{Al}_{0.9} \mathrm{Ga}{ }_{0.1} \mathrm{~N} / \mathrm{SiC}$ structure were studied. The $\mathrm{Al}_{0.9} \mathrm{Ga}_{0.1} \mathrm{~N} / \mathrm{SiC}$ photodiode showed a flat response in the deep-UV that exceeded $60 \%$ over a spectral range from $215-256 \mathrm{~nm}$, a significant enhancement in efficiency compared to a SiC PIN photodiode. 


\section{Chapter 5. $\mathrm{Al}_{0.8} \mathrm{Ga}_{0.2}$ As Avalanche Photodiodes for Near-UV detection}

In the previous chapters I discussed the performance of SiC-based APDs. They have exhibited decent external quantum efficiency from $200 \mathrm{~nm}$ to $350 \mathrm{~nm}$. However, due to the fact that $\mathrm{SiC}$ has an indirect bandgap the absorption coefficient is very small near the band edge. Several micrometers of absorption layer are needed to achieve strong absorption in the near UV, which will result in very high breakdown voltage and very long transient time. Among the other material candidates are Si, GaP and AlGaAs. Si APDS have, for most applications, been the preferred detectors owing to their high efficiency, low dark current, and low excess noise [5.1]. GaP APDs have demonstrated low dark current and high quantum efficiency. However, their excess noise is high as evidenced by the nearunity effective impact ionization coefficient ratio $\mathrm{k}(0.6 \sim 0.7)$ [5.2]. Another promising candidate is AlGaAs, which is used widely in semiconductor photonic devices, due to its tunable bandgap and refractive index.

\subsection{Material property}

There have been numerous published works on AlGaAs-based photodiode with Al fraction less than $60 \%$ [5.3-5.5]. However, due to their relatively narrow bandgap, the dark current is higher than that of the wide-bandgap materials discussed in previous chapters [5.6]. Also the low avalanche gain and poor impact ionization coefficient ratio limit their performance as avalanche photodiodes [5.7]. However, AlGaAs with a high Al mode fraction is a promising material candidate for high-performance APDs. Low impact ionization coefficient ratio between 0.1 and 0.2 has been reported for $\mathrm{Al}_{0.8} \mathrm{Ga}_{0.2} \mathrm{As}$ [5.8]. 
With $80 \% \mathrm{Al}$ mole fraction, the bandgap is $2.23 \mathrm{eV}$, which is expected to yield low dark current and a better absorption profile in the UV.

\subsection{Photodiode design and fabrication}

The first structure I investigated is shown in Figure 5-1. The doping of the p- layer that we requested from the foundry is as low as possible to ensure a fully depleted layer and uniform electrical field in the multiplication layer. The i layer thickness was designed to be $1 \mu \mathrm{m}$ in order to keep the breakdown electric field below that at which tunneling becomes significant. The top layer is GaAs since it is easier to form low resistance ohmic contact layer on $\mathrm{GaAs}$ than $\mathrm{Al}_{0.8} \mathrm{Ga}_{0.2} \mathrm{As}$. In order to ensure minimal absorption in the contact layer, its thickness is only $10 \mathrm{~nm}$.

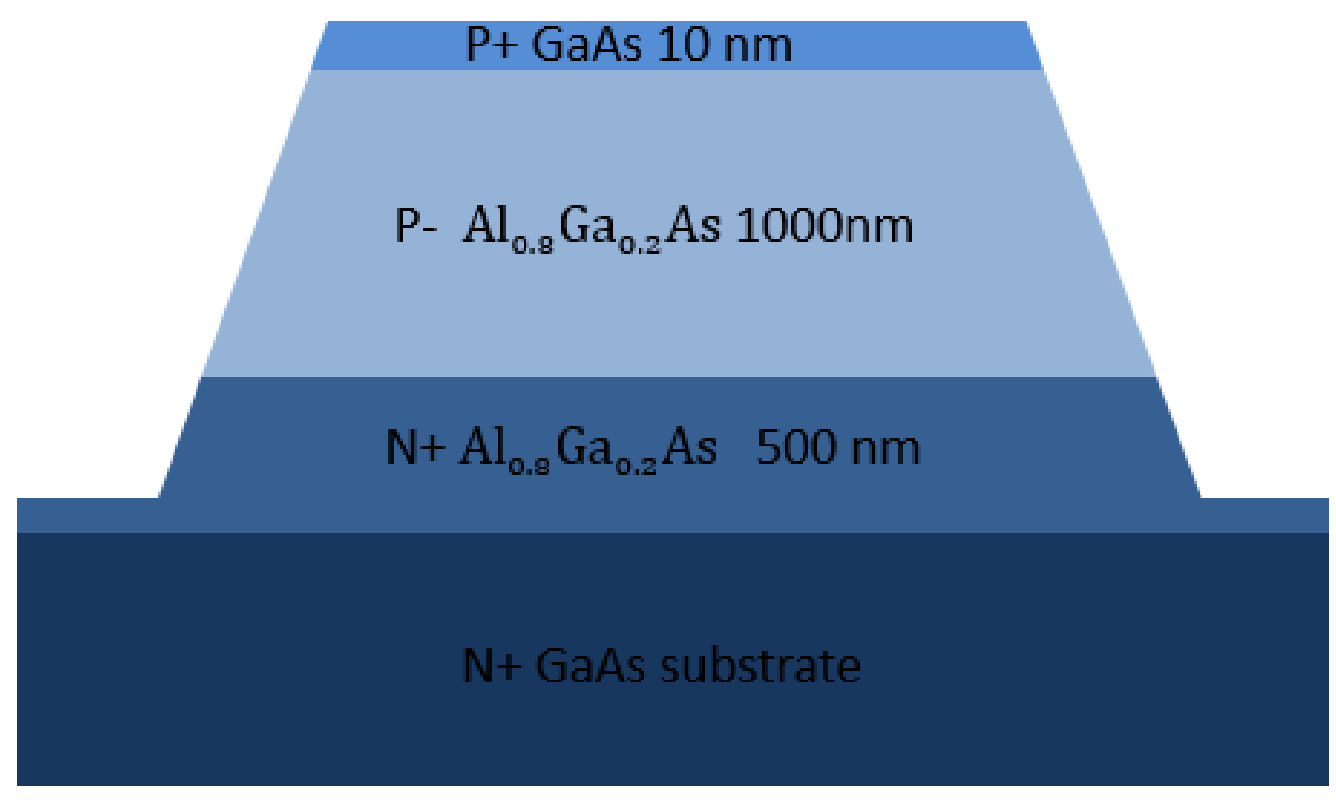

Figure 5-1. Cross section of the $\mathrm{Al}_{0.8} \mathrm{Ga} \mathrm{a}_{0.2} \mathrm{As}$ APD 
The $\mathrm{Al}_{0.8} \mathrm{Ga}_{0.2} \mathrm{As}$ APDs were fabricated using the process flow described in chapter 2 . The sidewall passivation is critical for $\mathrm{Al}_{0.8} \mathrm{Ga}_{0.2} \mathrm{As}$ detectors. For mesa structure APDs, atoms at the side wall that are not fully bonded will result in the formation of electrically active defects that degrade device performance through increased dark current or premature breakdown. In order to reduce the impact of these sidewall surfaces on device performance, mesa structure photodiodes are generally coated with a passivation material to reduce the density of active surface states. Sulfur passivation has been widely used to improve the electronic properties of GaAs devices by reducing the high density of states in the bandgap due to the native oxide layer, which is generally considered to be an unsuitable dielectric, having high defect densities [5.9]. The passivation procedure can cause unpinning of the Fermi level and inhibit the surface from further interaction with atmospheric contaminants. The sulfur passivation is typically achieved by soaking in $5 \%$ ammonium sulfide $\left((\mathrm{NH} 4)_{2} \mathrm{~S}_{\mathrm{x}}\right)$ solution before the deposition of $\mathrm{SiO}_{2}$ as sidewall passivation. This will leave a monolayer of sulfur that is bonded with the gallium atoms on the sidewall, without introducing any disorder or defects near the surface.

\subsection{Device performance}

The I-V characteristics of a $150 \mu \mathrm{m}$-diameter device are shown in Figure 5-2. The dark current is $31.7 \mathrm{pA}\left(179 \mathrm{nA} / \mathrm{cm}^{2}\right)$ when biased at $99 \%$ of the breakdown voltage, which yields a gain of 200. For comparison, a low-dark-current commercial Si APD from Laser Components, Inc. exhibits dark current density of $\sim 500 \mathrm{nA} / \mathrm{cm}^{2}$ at gain $=250$. In order to determine the primary effects that contribute to the dark current, the I-V characteristics of 
$\mathrm{Al}_{0.8} \mathrm{Ga}_{0.2} \mathrm{As}$ with different diameters were measured. The dark current at three different bias voltages is plotted versus device area in Figure 5-3. The linear relationship between the dark current of the device area at all the bias voltages implies that the dark current of these APDs is bulk-dominated both at low electric field and high electric field, which indicates that the sulfur passivation effectively suppresses surface leakage. For comparison, $150 \mu$ m-diameter devices were fabricated with the same process but without sulfur passivation. Figure 5-2 shows that the dark current with sulfur passivation is one order of magnitude lower than the one without sulfur passivation near breakdown.

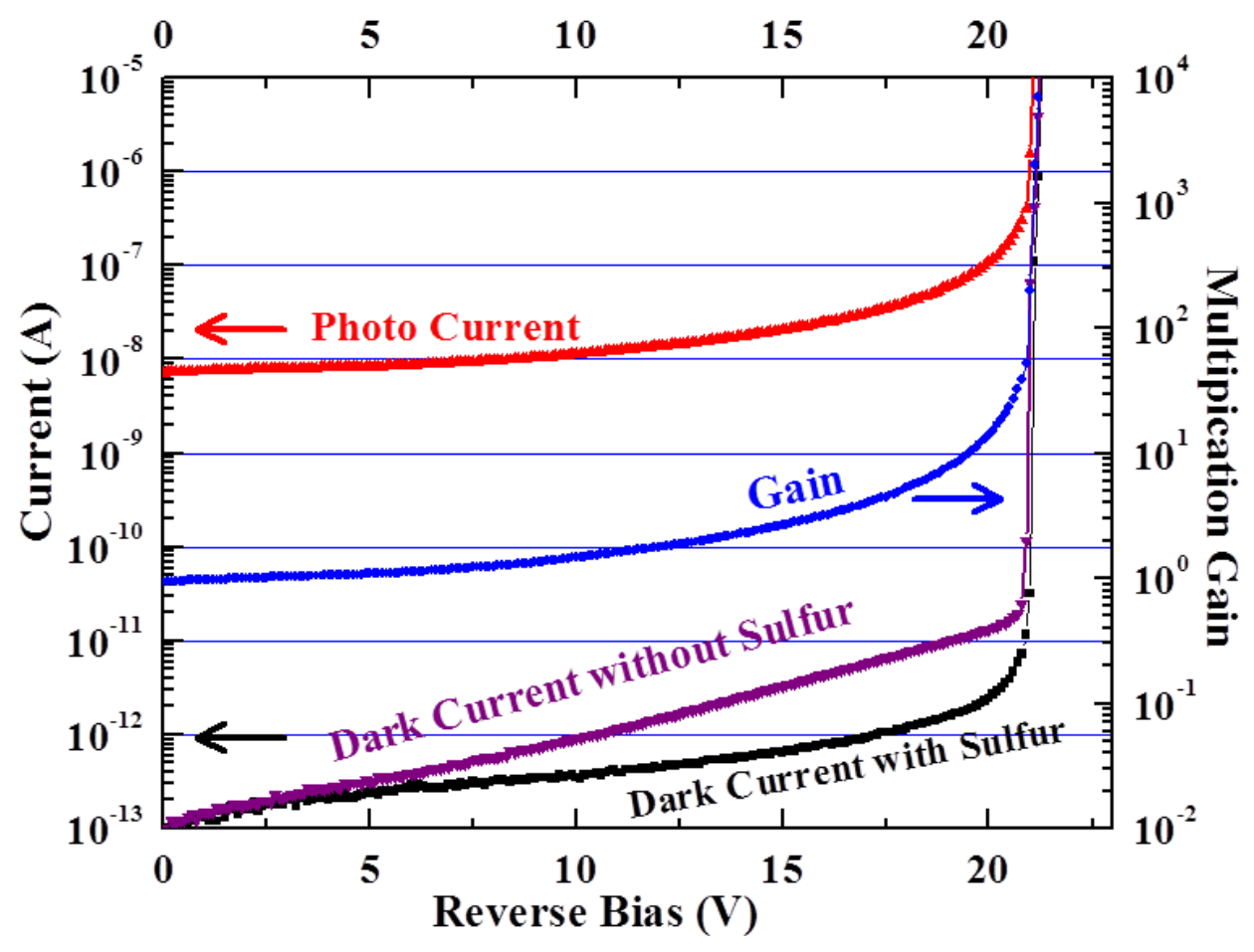

Figure 5-2. I-V characteristics of the $\mathrm{Al}_{0.8} \mathrm{Ga}$. 


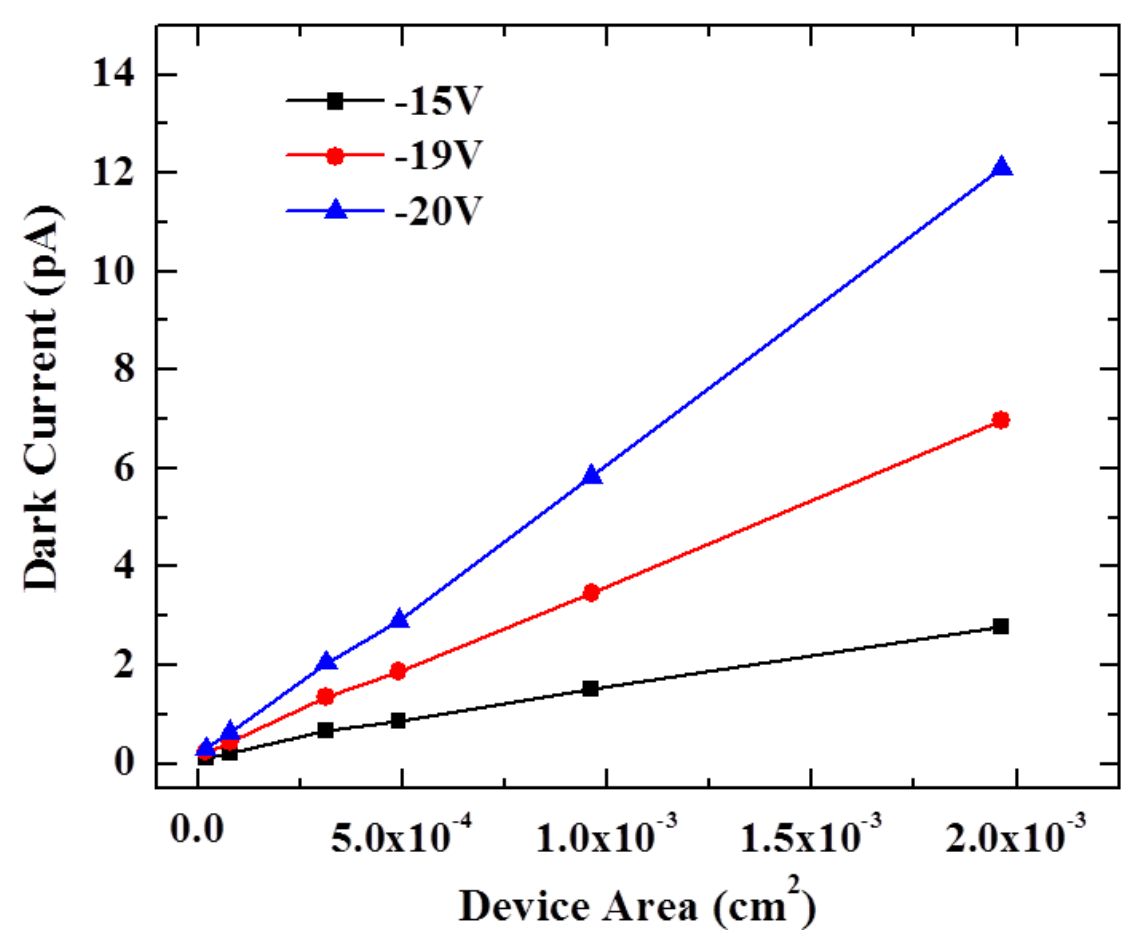

Figure 5-3. Dark current vs device area of Alo.8Ga0.2As APD

The external quantum efficiency at unity gain is plotted versus wavelength in Figure 54. The peak quantum efficiency is $27 \%$ at $465 \mathrm{~nm}$. The cut-off wavelength is approximately $550 \mathrm{~nm}$, which corresponds to the bandgap of $\mathrm{Al}_{0.8} \mathrm{Ga}_{0.2} \mathrm{As}$. However, an abrupt decrease in the efficiency dropping from $10 \%$ to $5 \%$ from $400 \mathrm{~nm}$ to $350 \mathrm{~nm}$ is observed, The relatively low external quantum efficiency can be explained by the capacitance measurement shown in Figure 5-6. The depletion width of the photodiode is calculated from the capacitance. The result shows that only $1 / 3$ of the $p$ - layer is depleted. A further SIMS analysis shown in Figure 5-6 revealed that the doping of the p- layer is $2 \times 10^{17} \mathrm{~cm}^{-}$ ${ }^{3}$, which is much higher than specified. The poor UV response is due the fact that a large fraction of the carriers were generated in the undepleted p- layer. Some carriers are generated as far as $600 \mathrm{~nm}$ away from the depletion region. Combined with short diffusion 
length, this results in poor efficiency. A simulation of the external quantum efficiency shown in Figure 5-4 yields a diffusion length of $120 \mathrm{~nm}$ for electrons in the p-doped $\mathrm{Al}_{0.8} \mathrm{Ga}_{0.2} \mathrm{As}$ layer, which is much shorter than the distance most electrons need to diffuse before reaching the edge of the depletion region.

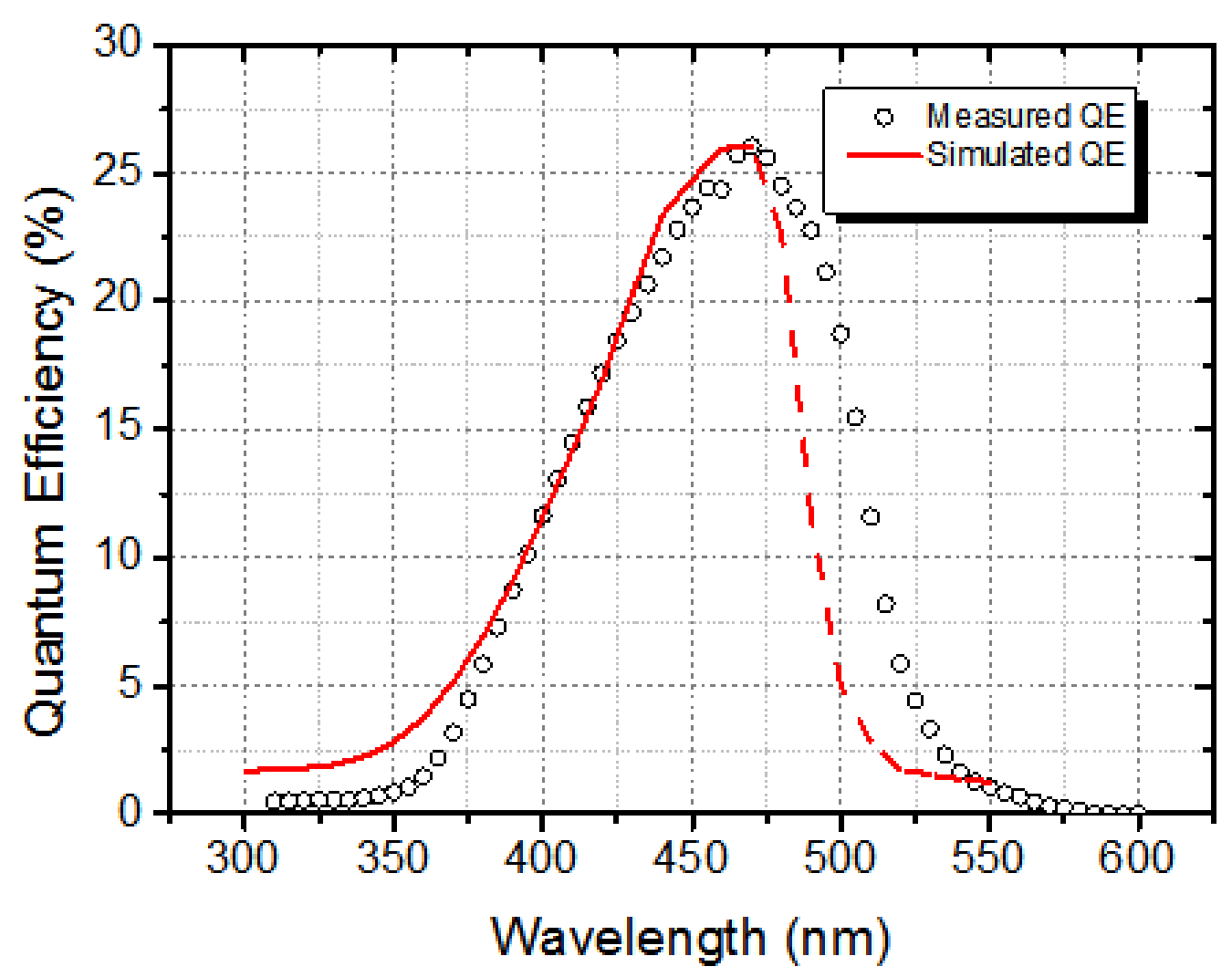

Figure 5-4. Measured and simulated external quantum efficiency of $\mathrm{Al}_{0.8} \mathrm{Ga}_{0.2} \mathrm{As}$ APD 


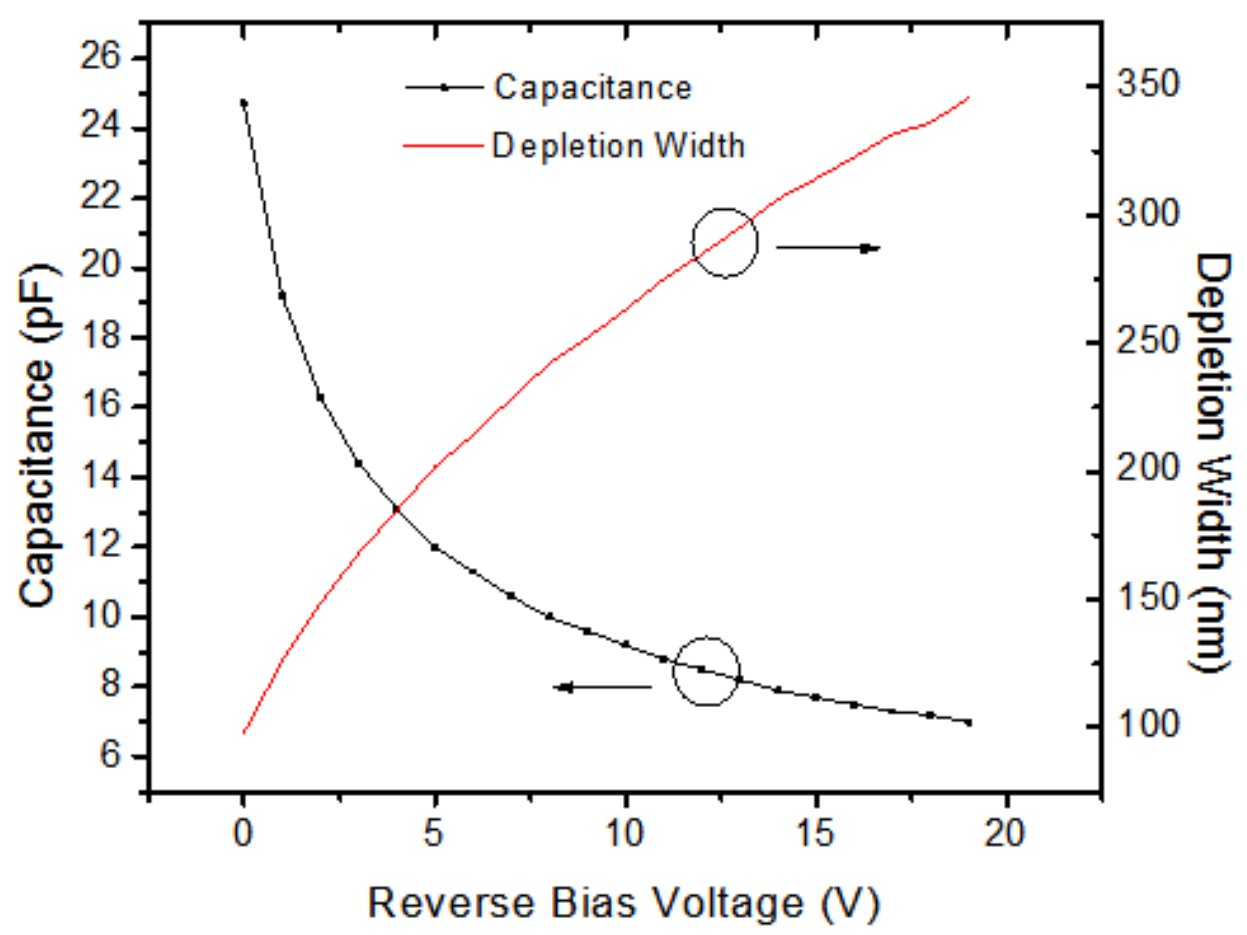

Figure 5-5. Capacitance and depletion width vs voltage of the $\mathrm{Al}_{0.8} \mathrm{Ga}_{0.2} \mathrm{As} \mathrm{APD}$

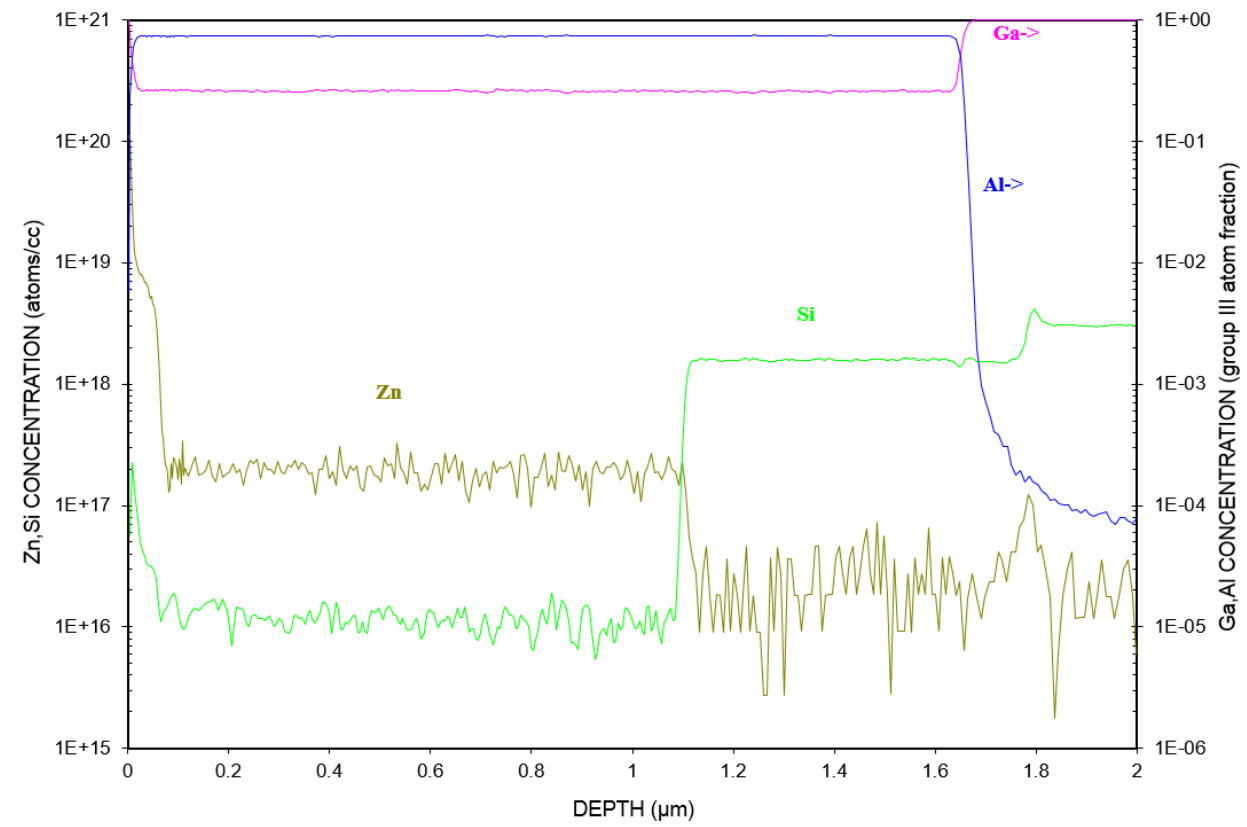

Figure 5-4. SIMS analysis of $\mathrm{Al}_{0.8} \mathrm{Ga} \mathbf{a}_{0.2} \mathrm{As}$ wafer 
The excess noise factor was measured following the procedure described in chapter 2 . The device was illuminated with an argon laser at 351/363 nm. Figure 3-16 shows a plot of the excess noise factor versus gain for the $\mathrm{Al}_{0.8} \mathrm{Ga}_{0.2} \mathrm{As}$ APD. The curves for $\mathrm{k}$ values ranging from 0.1 to 0.2 are included for reference. A $\mathrm{k}$ value of $\sim 0.15$ provided the best fit to the data. This measurement agrees well with previous reports on $\mathrm{Al}_{0.8} \mathrm{Ga}_{0.2} \mathrm{As}$ APD.

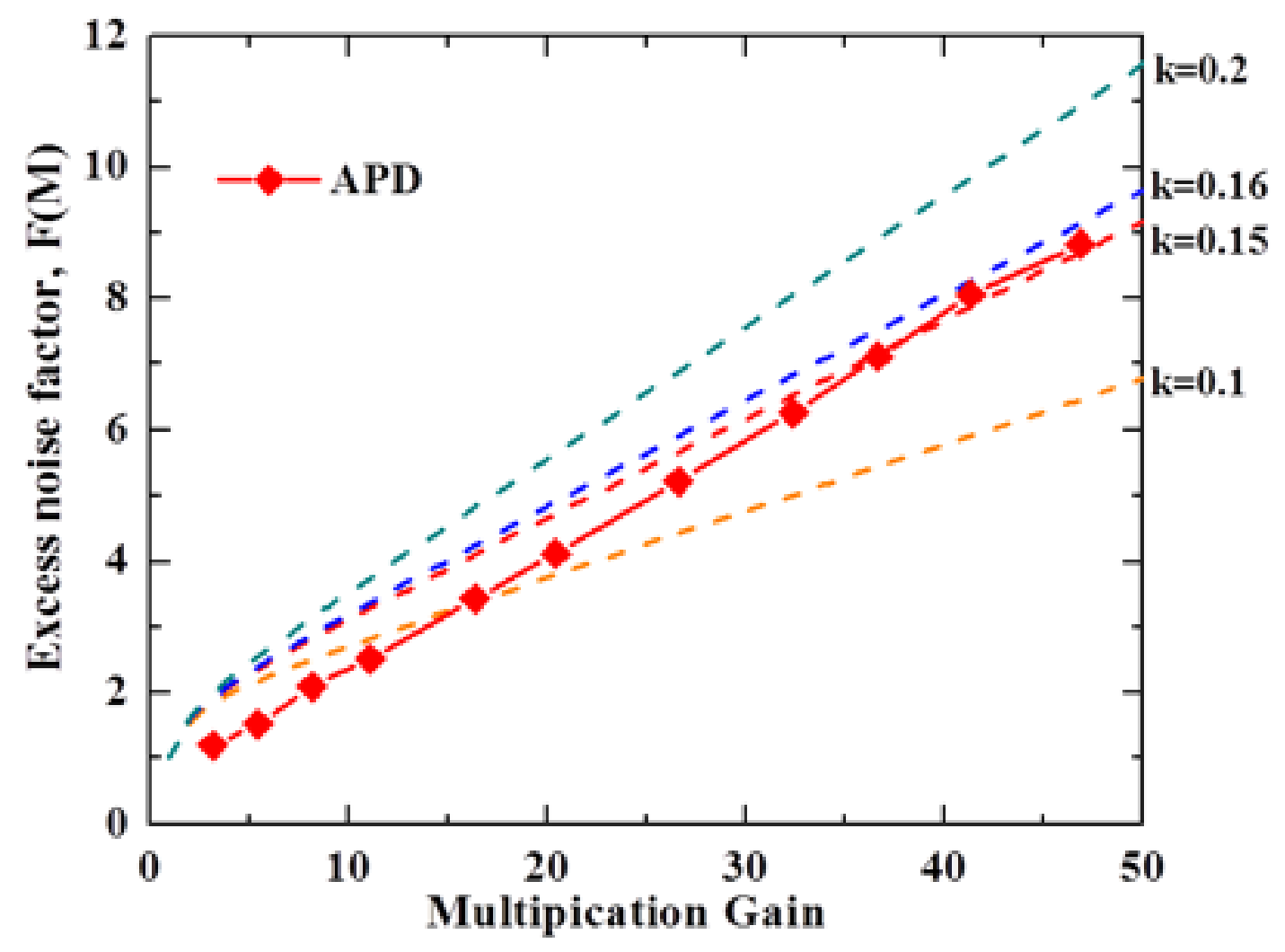

Figure 5-7. Excess noise of $\mathrm{Al}_{0.8} \mathrm{Ga}_{0.2 \mathrm{As}} \mathrm{APD}$

For the spatial uniformity measurements, an $\mathrm{Al}_{0.8} \mathrm{Ga}_{0.2} \mathrm{As}$ APD with diameter of 200 $\mu \mathrm{m}$ was biased near breakdown at a gain of 10. Light from an argon laser at 351/363 nm was scanned across the detector while the photocurrent was measured with a lock-in amplifier. The spot size was $\sim 5 \mu \mathrm{m}$, which is smaller than the contact ring of the device allowing us to resolve the features of the device. In Figure 5-5 the center region represents 
the active or operational region of the device. The valley directly outside the center region is the top contact ring. This feature is due to the fact that the metal of the contact ring blocks the incident signal. The outer ring is the edge of the mesa. Shadowing of the probe tip causes the dip in the upper region of the ring. It is observed from the raster scan that the $\mathrm{Al}_{0.8} \mathrm{Ga}_{0.2} \mathrm{As}$ APD has a very uniform spatial response.

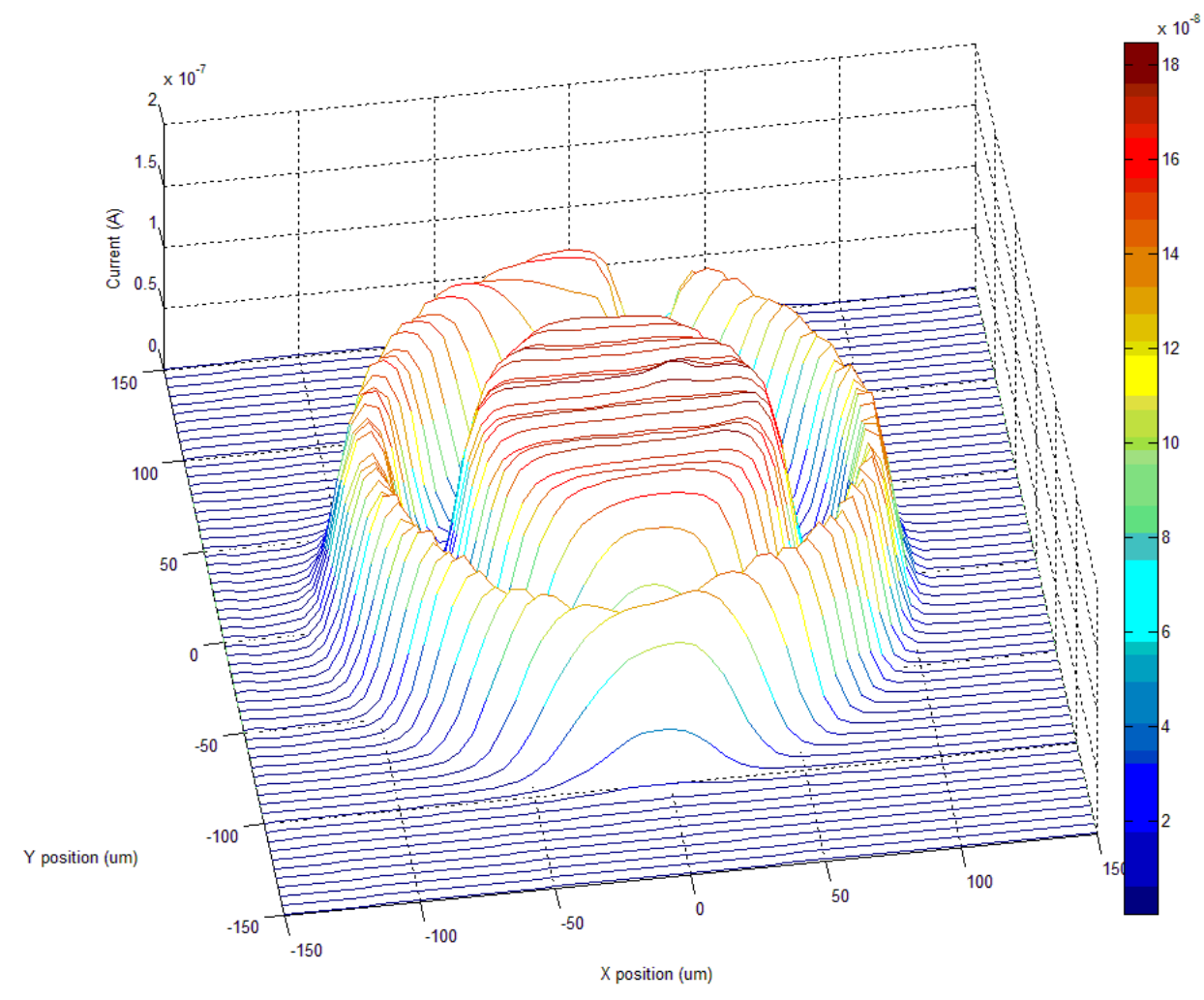

Figure 5-8. Raster scan $\mathrm{Al}_{0.8}$ Ga0.2As APD at gain = 10

\subsection{Conclusion}

In this chapter, $\mathrm{Al}_{0.8} \mathrm{Ga}_{0.2} \mathrm{As}$ APD was studied as a candidate to replace $\mathrm{Si}$ APDs for the weak light detection in the near-UV spectrum. $\mathrm{Al}_{0.8} \mathrm{Ga} \mathrm{G}_{0.2} \mathrm{As}$ APDs with PIN structure were 
designed, fabricated and characterized. Sulfur passivation using 5\% ammonium sulfide was used to passivate the sidewall of the photodiode. The dark current of the $\mathrm{Al}_{0.8} \mathrm{Ga} \mathrm{a}_{0.2} \mathrm{As}$ APD was $31.7 \mathrm{pA}\left(179 \mathrm{nA} / \mathrm{cm}^{2}\right)$ at gain of 200 , which is lower than the state of the art $\mathrm{Si}$ APD. The dark current of the photodiode was found to be proportional to the device area, which indicates that the dark current is bulk dominated and the sulfur passivation is effective. The excess noise of the $\mathrm{Al}_{0.8} \mathrm{Ga}_{0.2} \mathrm{As}$ APD is low with a $\mathrm{k}$ value of $\sim 0.15$. The raster scan at a gain of 10 showed a uniform spatial response.

The $\mathrm{Al}_{0.8} \mathrm{Ga}_{0.2} \mathrm{As}$ APD yielded a peak quantum efficiency of $27 \%$ at $465 \mathrm{~nm}$. However, an abrupt decrease of the efficiency at shorter wavelength is observed, decreasing from $10 \%$ to $5 \%$ for wavelengths $400 \mathrm{~nm}$ to $350 \mathrm{~nm}$. This is due to the fact that the doping in the p- layer is higher than the design. A large fraction of the carriers were generated in the undepleted p- layer. A lower doping in the lightly-doped layer is needed to achieve higher quantum efficiency in the near-UV spectrum. 


\section{Chapter 6. GaAs/AlGaAs Photodiode for Wide Spectrum Detection}

GaAs photodetectors are widely used in for visible and near infrared detection [6.1]. However, there have been few reports on the performance in the UV spectrum. In this chapter, I will discuss different techniques to enhance the UV response of the GaAs photodiode. In this project I developed a photodiode that can achieve high quantum efficiency from UV to near infrared. This work was funded by NASA and motivated by the goal of reducing the number of cameras in some satellites.

\subsection{Wafer Design}

A schematic cross section of the first structure studied is shown in Figure 6-1. The wafer was purchased from Landmark Optoelectronics, Inc. The i-layer thickness was chosen to ensure strong absorption for wavelengths as long as $850 \mathrm{~nm}$. The Al concentration in the AlGaAs layer is graded from $30 \%$ at the top to $10 \%$ at the AlGaAs/GaAs interface. This graded bandgap structure creates an electric field that assists carrier transport into the depletion region instead of relying exclusively on diffusion. 


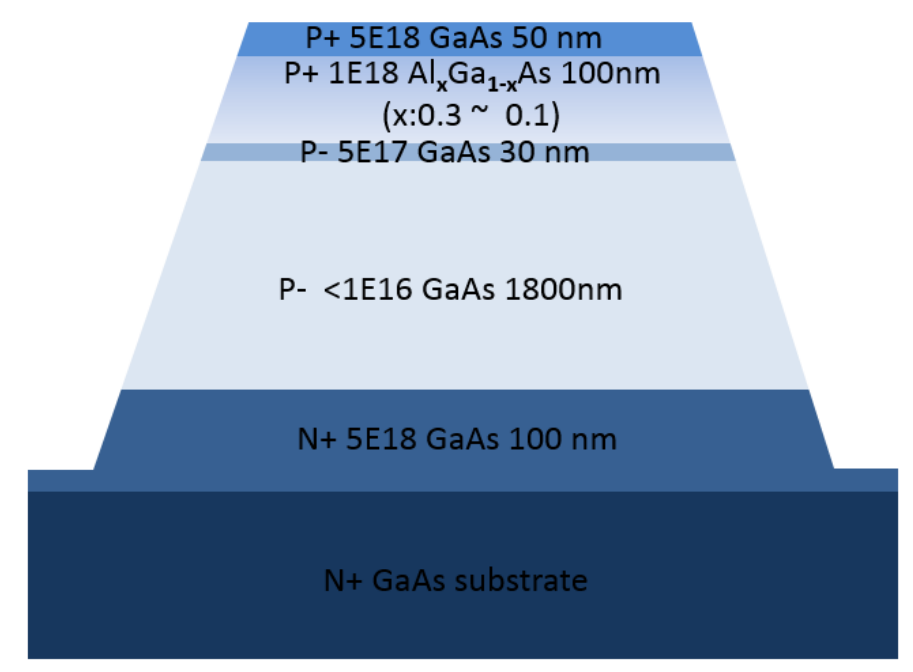

Figure 6-1. Wafer structure for GaAs/AlGaAs photodiode

GaAs/AlGaAs photodiodes were fabricated using the process flow described in chapter 2. The current-voltage (I-V) characteristics of a $200-\mu \mathrm{m}$ diameter device are shown in Figure 6-2. The photodiode has a breakdown voltage of $50 \mathrm{~V}$. The dark current is less than $3 \mathrm{pA}$ for bias voltage lower than $30 \mathrm{~V}$. The dark current near breakdown at a gain of 10 is $500 \mathrm{pA}$.

The initial measurement of the external quantum efficiency yielded low efficiencies in the UV spectrum. This is due to two reasons. First, the absorption depth in this spectrum region is very shallow. Most of the photons are absorbed within $100 \mathrm{~nm}$ of the surface. Owing to surface recombination and the short diffusion length, a significant fraction of the carriers recombine prior to being collected. Second, the reflectance of GaAs increases rapidly with the photon energy. For photons with wavelength shorter than $400 \mathrm{~nm}$, the reflectance is higher than $40 \%$.

In order to improve the quantum efficiency in the UV spectrum, the recessed window structure and surface texturing techniques were investigated. 


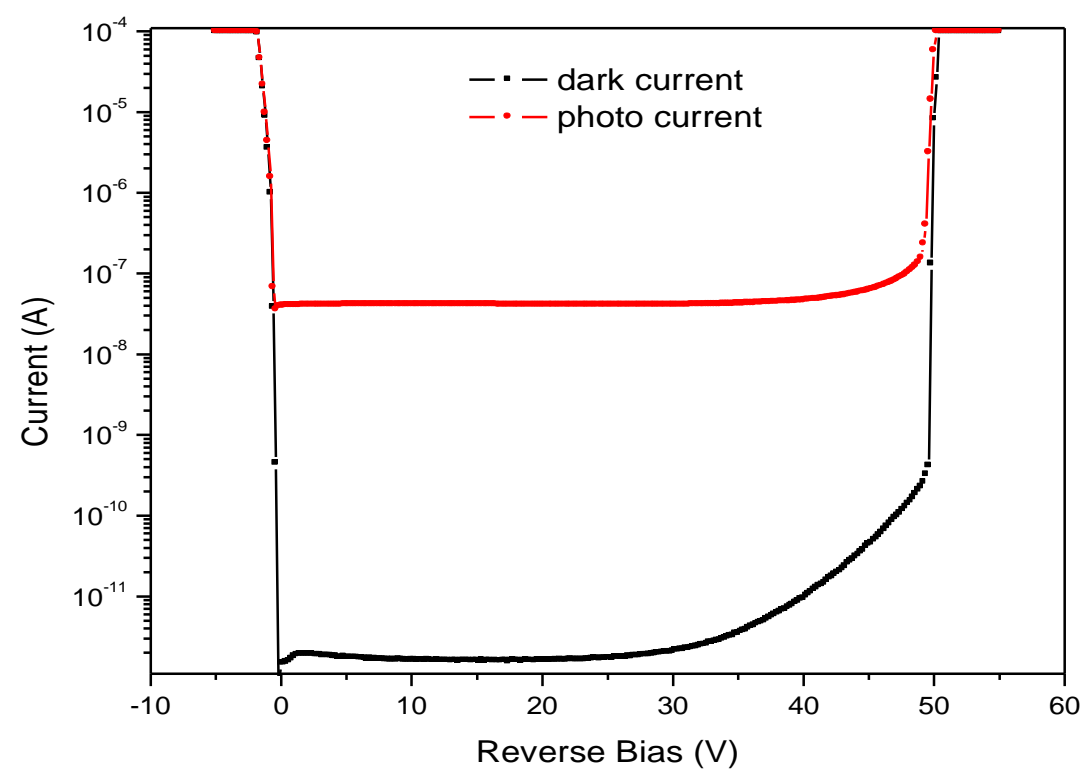

Figure 6-2. Current - voltage characteristics of a $200 \mu \mathrm{m}$-diameter GaAs/AlGaAs photodiode

\subsection{Recessed window structure}

For the recessed window approach, the top GaAs p+ cap layer was removed by chemical etching in the active portion of the device. The etch process was optimized such that the bottom of the window extended into the $100 \mathrm{~nm}$ p AlGaAs layer. SEM top-view and side-view photographs after etching the window are shown in Figure 6-3. The recessed window enhances detection at short wavelengths by decreasing the distance to the collection region, which is beneficial for UV radiation. 


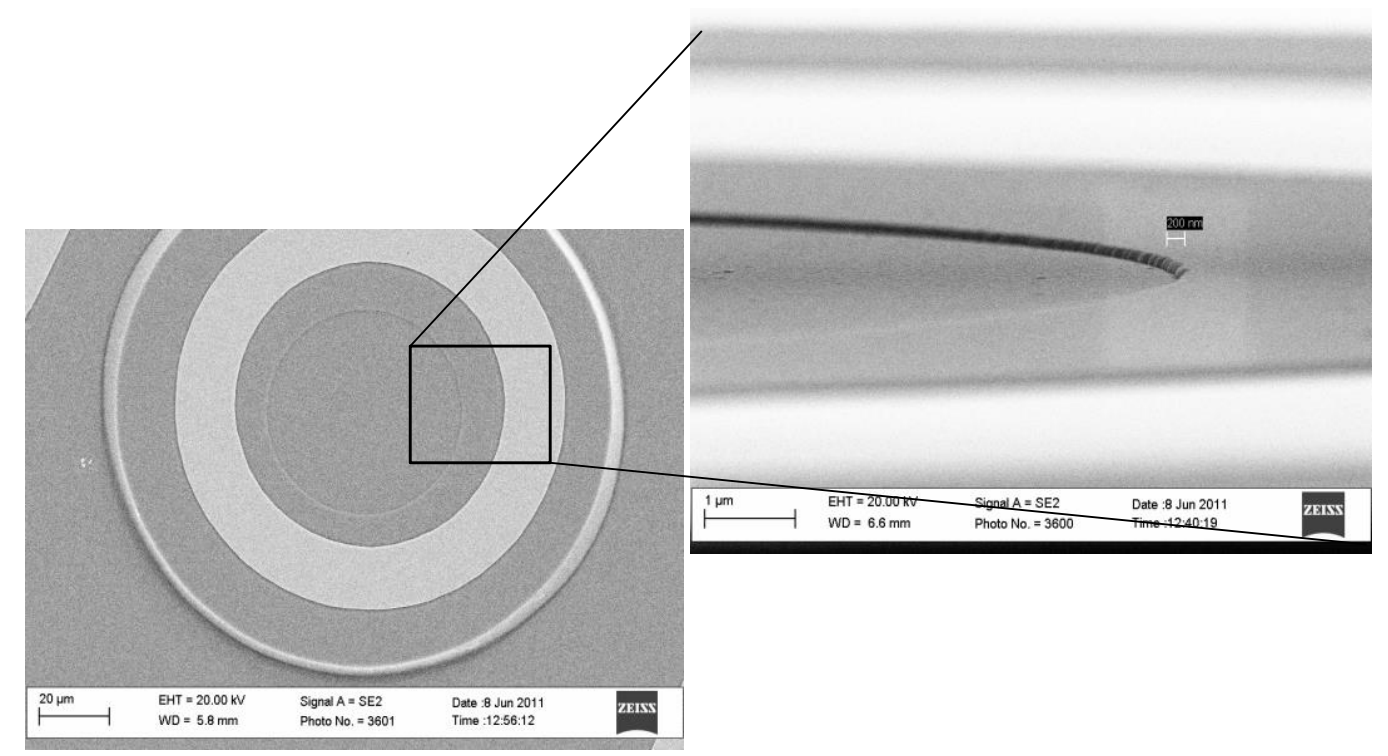

Figure 6-3. SEM image of the recessed window on GaAs/AIGaAs photodiode

\subsection{Nanosphere surface texturing}

At present, dielectric anti-reflection (AR) coatings are widely used to reduce the surface reflectivity and increase the responsivity of photodiode near the wavelength of interest. However, the reflectance of an AR coating is very sensitive to the wavelength and thus not able to achieve low reflectance across a wide spectrum. Surface texturing is another technique to reduce the surface reflectance loss. It has been demonstrated to successfully reduce reflectance in solar cells, light emitting diodes, and photodiodes [6.2-6.5]. A previous student in our group has investigated laser surface texturing on Si photodiodes. High efficiency over a broad spectrum was achieved [6.6]. However, the devices exhibited higher dark current after the texturing, possibly due to the laser induced damage. Alternatively, a texturing technique referred to as nanosphere natural lithography texturing can create a random pattern with feature size $\sim 100 \mathrm{~nm}$, while limiting the damage to the top contact layer [6.7]. 
For the texturing, $5 \%$ solution of $100 \mathrm{~nm}$-diameter $\mathrm{SiO}_{2}$ spheres in water was purchased from Corpuscular Inc. A solution of surfactant Triton X-100 and Methanol was added to the suspension. The volume ratio of the $\mathrm{SiO}_{2}$ suspension to Triton $\mathrm{X}-100$ solution was 10:1. The $\mathrm{SiO}_{2}$ suspension was spun onto the wafer with spin rate of $100 \mathrm{rpm}$. This created a uniform monolayer of $\mathrm{SiO}_{2}$ spheres that covered most of the surface area after the solvent evaporated. Then an $\mathrm{Ar} / \mathrm{BCl}_{3}$ inductive coupled plasma etch was performed to transfer the nanosphere monolayer random pattern onto the wafer surface. The etch depth was $~ 100$ $\mathrm{nm}$ in the areas between the $\mathrm{SiO}_{2}$ spheres. This depth kept the plasma from etching into the lightly-doped layer. A buffered oxide etch was used to remove any $\mathrm{SiO}_{2}$ residue. A welltextured surface will look very dark. A scanning electron microscope (SEM) image of the textured surface is shown in Figure 6-4. The reflectance of the textured surface was measured using the Perkin Elmer Lambda 950 spectrometer. The detailed measurement steps are discussed in Chapter 2. Figure 6-5 compares the spectral reflectance of the textured GaAs/AlGaAs wafer and the $\mathrm{SiO}_{2} \mathrm{AR}$ coating deposited on the same wafer using PECVD. The AR coating was optimized for $\sim 400 \mathrm{~nm}$. The minimum reflectance for the AR coating is the same as that for the textured surface. However, the textured surface provides low reflectivity across a very wide spectrum from $300 \mathrm{~nm}$ to $600 \mathrm{~nm}$. 


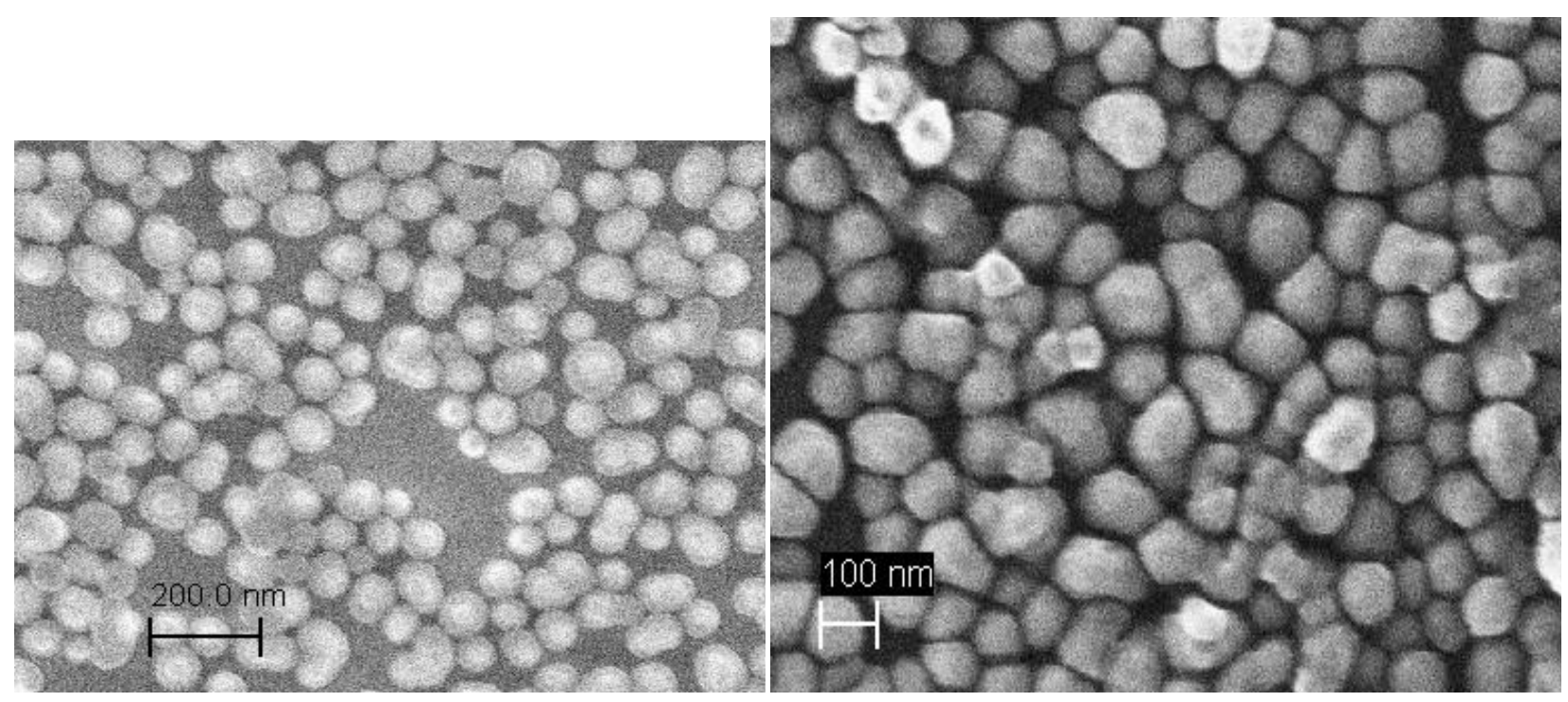

Figure 6-4. SEM image of the textured surface on GaAs/AIGaAs photodiode

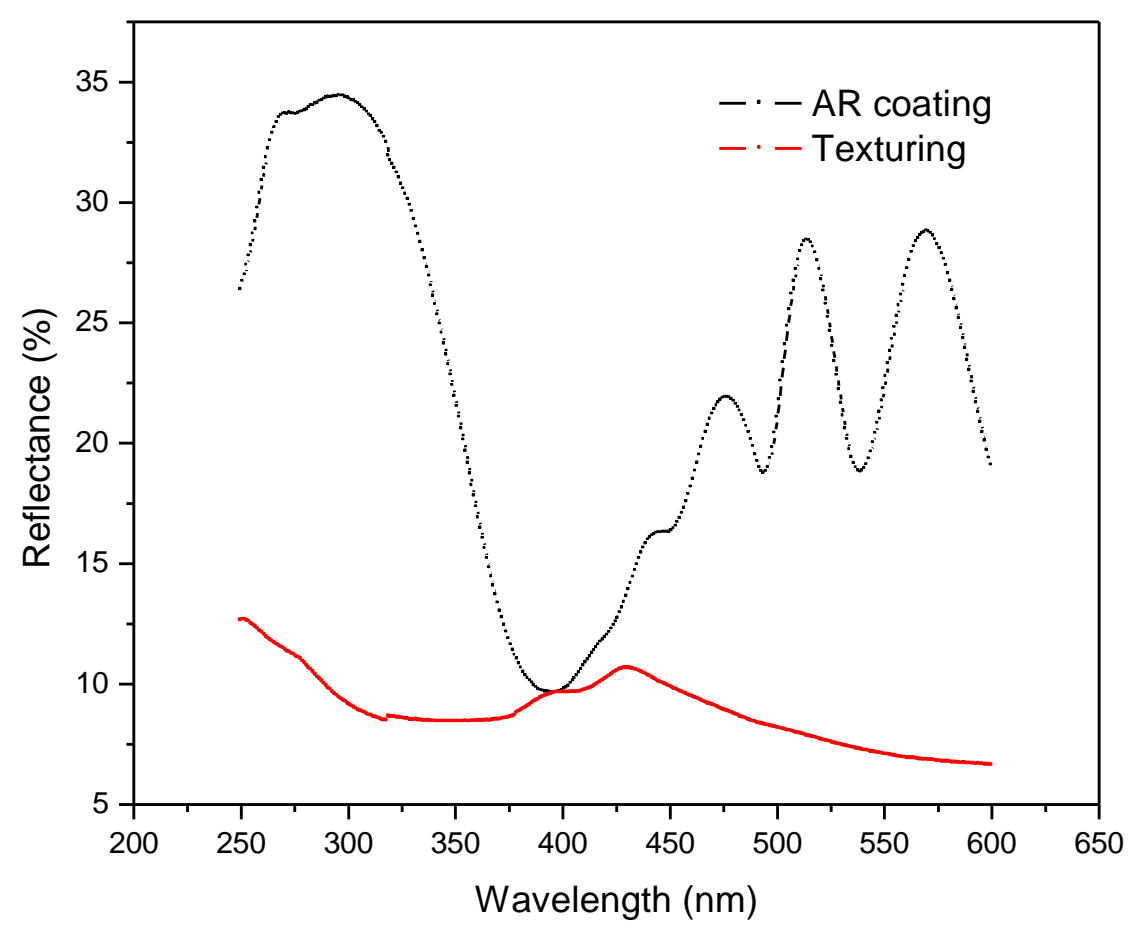

Figure 6-5. Measured reflectance of the textured surface compared with $\mathrm{SiO}_{2} \mathrm{AR}$ coating 


\subsection{Device performance}

The GaAs/AlGaAs photodiodes were fabricated with both the recessed window and surface texturing technique using the processing steps described in chapter 2 . The recessed window and surface texturing were finished before the mesa patterning so that the ICP plasma would not damage the sidewall. The same I-V characteristics were achieved after incorporating the surface texturing, showing that the surface texturing did not increase the dark current of the photodiode. The external quantum efficiency of the GaAs/AlGaAs photodiode with recessed window and surface texturing is plotted in Figure 6-6 and compared with a photodiode that was fabricated without these techniques. A much flatter curve with efficiency over $45 \%$ across a wide spectrum from $350 \mathrm{~nm}$ to $850 \mathrm{~nm}$ was achieved. The external quantum efficiency at $400 \mathrm{~nm}$ increased from 5\% to $45 \%$.

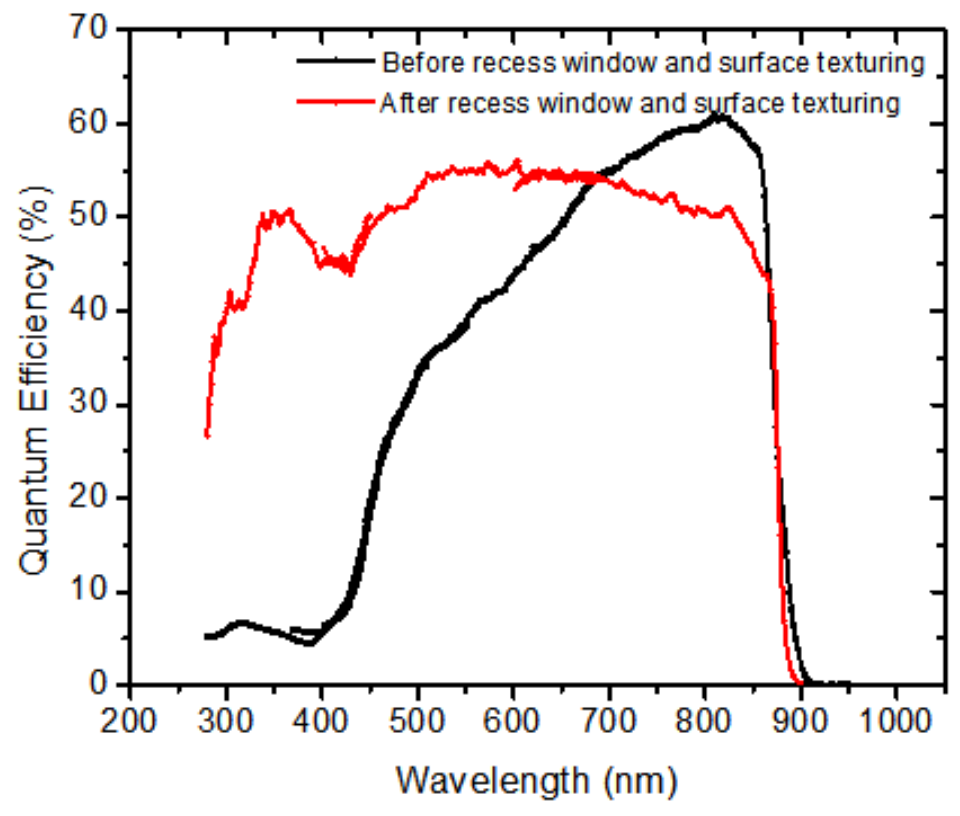

Figure 6-6. External quantum efficiency of GaAs/AIGaAs photodiode 


\subsection{High-efficiency UV to near IR arrays}

The photodiodes described in the previous section represent new state-of-the-art sensitivity across a broad spectral range. Given this success, these devices were fabricated into $1 \times 16$ and $1 \times 32$ photodiode arrays. For the array fabrication, new photomask was designed using a round-corner square mesa in order to achieve higher fill factor. The current voltage characteristics for each element in the array were measured. Figure 6-7(a) is a photograph of a completed 1x16 linear array and Fig. 6-7(b) is an enlarged picture of three $200 \mu \mathrm{m} \times 180 \mu \mathrm{m}$ diodes. Figure 6-8 shows the dark current of each of the 16 photodiodes at $-1 \mathrm{~V}$ bias. From the dark current distribution we can find the uniformity among the elements in the linear array is very good. The dark current of the single element ranges from $0.7 \mathrm{pA}$ to $3.4 \mathrm{pA}$. Figures 6-11 and 6-12 show similar results for the $1 \mathrm{x} 32$ linear arrays. Because of the smaller size of the element in the 1 x 32 array, the dark current for the element is lower and better uniformity is achieved. 


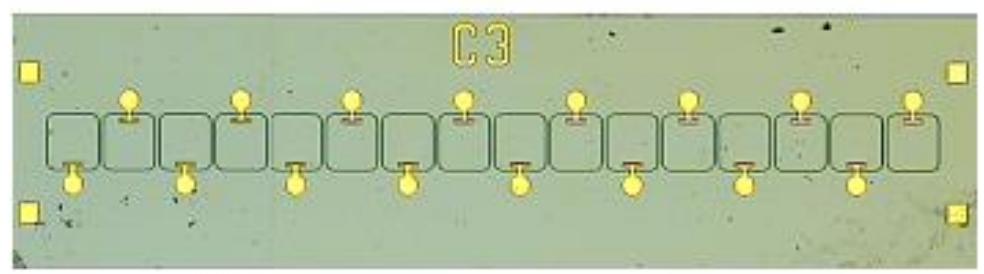

(a)

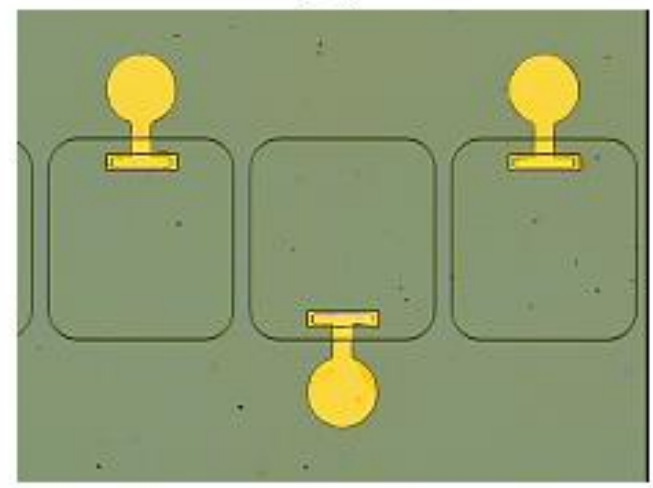

(b)

Figure 6-7. (a) Photograph of a $1 \times 16$ UV to near IR linear array and (b) enlarged picture of three $200 \mu \mathrm{m} x$ $180 \mu \mathrm{m}$ diodes.

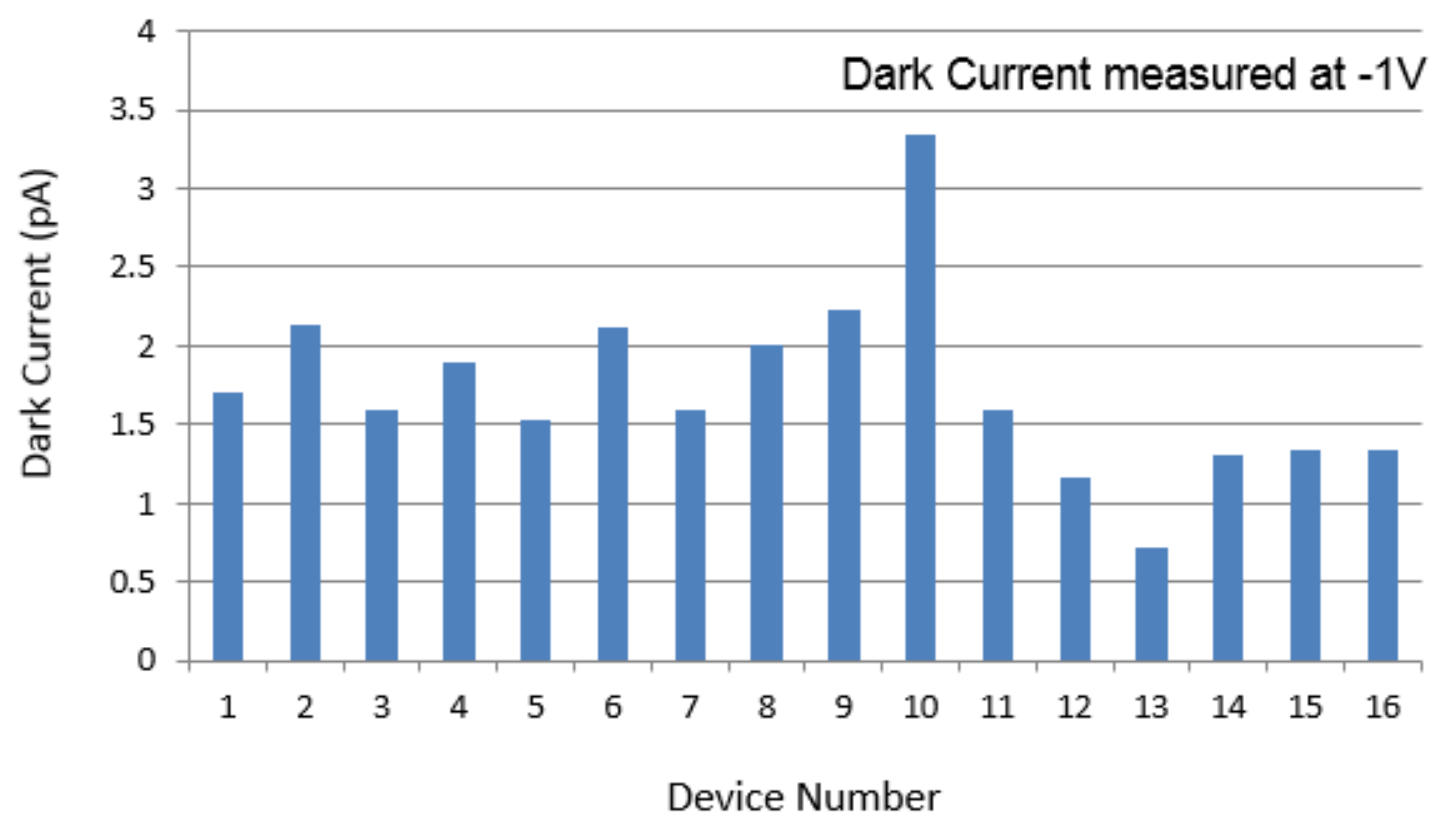

Figure 6-8. Dark current of each element in the 1x16 UV to near IR linear array at -1 V bias. 


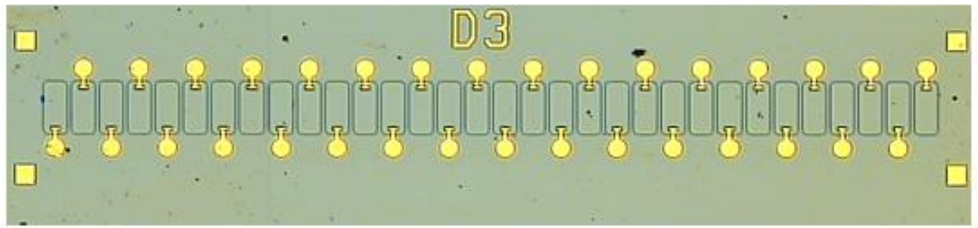

(a)

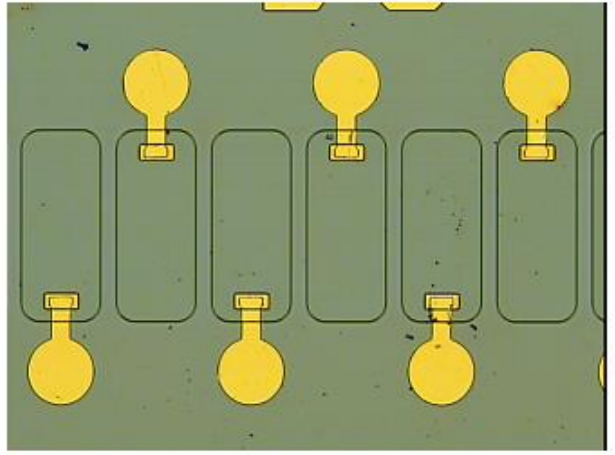

(b)

Figure 6-9. (a) Photograph of a 1x32 UV to near IR linear array and (b) enlarged picture of three $200 \mu \mathrm{m} x$ $85 \mu \mathrm{m}$ diodes.

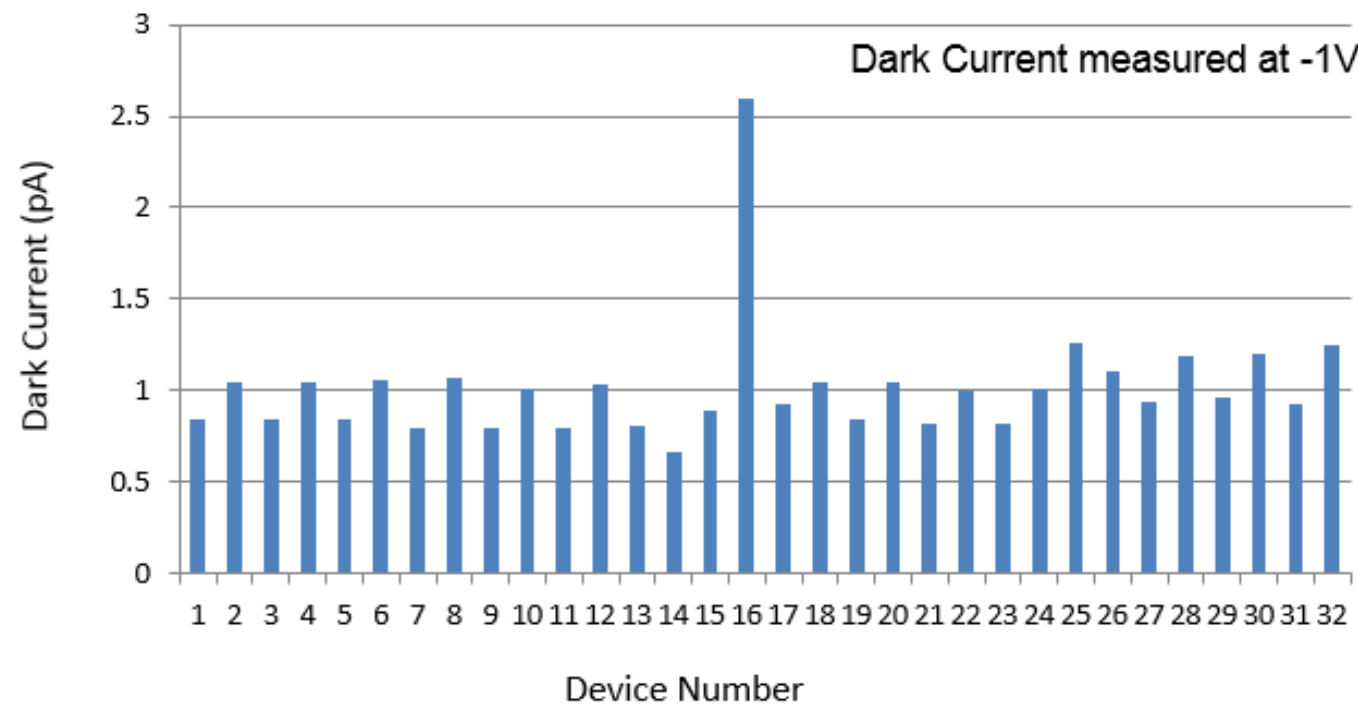

Figure 6-10. Dark current of each element in the 1x32 UV to near IR linear array at -1 V bias. 


\subsection{Conclusion}

GaAs/AlGaAs photodiodes were studied for wide spectrum detection from the nearUV to the near infrared. The photodiodes exhibited low dark current of less than $10 \mathrm{pA}$ at bias voltage lower than $30 \mathrm{~V}$ and $500 \mathrm{pA}$ near breakdown at gain of 10 .

In ordered to enhance the near-UV response and achieve a flat quantum efficiency curve over the wide spectrum, a recessed window and surface texturing were employed. In the recessed window structure, part of the highly doped layer in the active region is removed by chemical etching. The recessed depth was optimized such that the bottom of the window extended into the $100 \mathrm{~nm}$ p AlGaAs layer. Following the recessed window, the surface was textured using the nanosphere natural lithography etching, which created a random pattern on the top surface with feature size of $\sim 100 \mathrm{~nm}$. The etch depth was controlled at around $100 \mathrm{~nm}$ to keep prevent etching the lightly-doped layer.

With recessed window and surface texturing, the GaAs/AlGaAs photodiode yielded external quantum efficiency over $45 \%$ from $350 \mathrm{~nm}$ to $850 \mathrm{~nm}$. At $400 \mathrm{~nm}$, the responsivity showed relative enhancement of $300 \%$. These photodiodes were fabricated into 1x16 and 1x32 UV-to-near infrared linear arrays and delivered to NASA. 


\section{Chapter 7. Conclusion and future work}

\subsection{Summary}

My work has focused on developing high performance avalanche photodiodes (APD) in the deep-UV $(200 \mathrm{~nm}-250 \mathrm{~nm})$ and near-UV $(300 \mathrm{~nm}-400 \mathrm{~nm})$ spectrum for weak light detection.

Previously in our group, 4H-SiC APDs with low dark current, low noise, high gain and high peak quantum efficiency have been developed. However, the narrow spectral response has limited their use for applications in the deep-UV and near-UV detection. Initially I studied the spectral response of the SiC APD with PIN structure by modeling the external quantum efficiency. The results showed that over $50 \%$ of the carriers that were generated in the highly-doped top layer were did not diffuse into the depletion region, which resulted in poor deep-UV response. This is due to the fact that the diffusion length in highly-doped $\mathrm{SiC}$ is relatively short and the surface recombination velocity is high. Different approaches utilizing both the homojunction and heterojunction structures to enhance the deep-UV response of the SiC APD have been reported here.

A SiC metal-n- ${ }^{-}$p photodiode was first studied in order to minimize the absorption in the undeleted region. The fabricated device demonstrated flat external quantum efficiency of $\sim 45 \%$ from $270 \mathrm{~nm}$ to $225 \mathrm{~nm}$ with a slight decrease below $225 \mathrm{~nm}$. This response is greater than that observed for the p-n-n+ diodes for wavelengths shorter than $240 \mathrm{~nm}$ despite losses due to the semi-transparent metal contact. The deep-UV response enhancement resulted from the improved collection of photo-generated carriers through 
drift within the high electric field region near the surface of the lightly-doped SiC absorption region despite the presence of surface states.

A SiC NIP photodiode with graded doping profile in the top $\mathrm{n}$ layer was also investigated. The goal of this structure was to utilize the high-low junction to provide an effective electric field that can stop the carriers from reaching the top. An enhanced deepUV response was observed with the external quantum efficiency of $\sim 30 \%$ at $240 \mathrm{~nm}$.

The AlGaN/SiC SAM structure illustrates another promising way to achieve high, tunable UV response with an AlGaN absorption layer. An AlGaN/AlN/SiC SAM structure photodiode with $\mathrm{Al}_{0.62} \mathrm{Ga}_{0.38} \mathrm{~N}$ as the absorption layer was fabricated and characterized. An AlN layer proved to be effective in reducing the polarization-induced charge and extending the electric field into the AlGaN. However the bias dependent behavior of the $\mathrm{AlGaN} / \mathrm{AlN} / \mathrm{SiC}$ indicated that the AlN acts as a barrier layer, thus a further optimization of the AlN layer thickness was needed.

A $\mathrm{SiC}$ detector with $\mathrm{AlGaN}$ transparent window layer achieved high deep-UV response by absorbing the deep-UV photons in the depleted $\mathrm{SiC}$ layer. The $\mathrm{Al}_{0.8} \mathrm{Ga}_{0.2} \mathrm{~N} / \mathrm{AlN} / \mathrm{SiC}$ photodiodes exhibited a peak quantum efficiency of $\sim 80 \%$ at $242 \mathrm{~nm}$. A modified structure $\mathrm{Al}_{0.9} \mathrm{Ga}_{0.1} \mathrm{~N} / \mathrm{SiC}$ structure without the AlN layer was shown to further enhance the deepUV response, The $\mathrm{Al}_{0.9} \mathrm{Ga}_{0.1} \mathrm{~N} / \mathrm{SiC}$ photodiode showed a flat response in the deep-UV that exceeded $60 \%$ over a spectral range from $215-256 \mathrm{~nm}$, which is a significant enhancement compared with a SiC PIN photodiode

For near-UV detection, GaAs/AlGaAs was investigated as an material candidate to replace Si. $\mathrm{Al}_{0.8} \mathrm{Ga}_{0.2} \mathrm{As}$ APDs with PIN structure were designed, fabricated and characterized. Sulfur passivation using the 5\% ammonium sulfide was used to passivate 
the sidewall of the photodiode. The dark current of the $\mathrm{Al}_{0.8} \mathrm{Ga}_{0.2} \mathrm{As}$ APD was $31.7 \mathrm{pA}$ (179 $\mathrm{nA} / \mathrm{cm}^{2}$ ) at gain of 200, which is lower than state-of-the-art Si APDs. The dark current of the photodiode was found to be proportional to the device area, which indicates that the dark current is bulk dominated and the sulfur passivation is effective. The excess noise of the $\mathrm{Al}_{0.8} \mathrm{Ga}_{0.2} \mathrm{As}$ APD is low with a $\mathrm{k}$ value of $\sim 0.15$. The raster scan at a gain of 10 showed a uniform spatial response. The $\mathrm{Al}_{0.8} \mathrm{Ga}_{0.2} \mathrm{As}$ APD yielded a peak quantum efficiency of $27 \%$ at $465 \mathrm{~nm}$. However, an abrupt decrease of the efficiency from $10 \%$ to $5 \%$ for 400 $\mathrm{nm}$ to $350 \mathrm{~nm}$ was observed. This is due to the fact that the doping in the $\mathrm{p}$ - layer is higher than the design. A large fraction of the carriers were generated in the undepleted p- layer. A lower doping in the lightly-doped layer is needed to achieve higher quantum efficiency in the near-UV spectrum.

A GaAs/AlGaAs photodiode was studied for wide spectrum detection from the nearUV to the near infrared. The photodiodes exhibited low dark current of less than $10 \mathrm{pA}$ at bias voltage lower than $30 \mathrm{~V}$ and about $500 \mathrm{pA}$ near breakdown at gain of 10 . In ordered to enhance the near-UV response and achieve a flat quantum efficiency over a broad spectrum, recessed window and surface texturing were employed. In the recessed window structure, part of the highly doped layer in the active region was removed by chemical etching. The recessed depth was optimized such that the bottom of the window extended into the $100 \mathrm{~nm}$ p AlGaAs layer. Following the recessed window, the surface was textured using the nanosphere natural lithography etching, which created a random pattern on the top surface with feature size of about $100 \mathrm{~nm}$. The etching depth was controlled to approximately $100 \mathrm{~nm}$ in order to protect the lightly-doped layer. With the recessed window and surface texturing, the GaAs/AlGaAs photodiode yielded external quantum 
efficiency over $45 \%$ from $350 \mathrm{~nm}$ to $850 \mathrm{~nm}$. At $400 \mathrm{~nm}$, the responsivity improvement was $300 \%$. These photodiodes were fabricated into $1 \times 16$ and $1 \times 32$ arrays that exhibited excellent device-to-device uniformity.

\section{2 $\quad$ Future work}

\subsubsection{Nitrogen passivation on $\mathrm{SiC}$ avalanche photodiode}

As discussed in chapter 2, surface band bending is detrimental to the deep-UV response of $\mathrm{SiC}$. Different approaches have been investigated in chapter 2 and chapter 3 to overcome this problem. I have shown with the $\mathrm{SiC}$ metal-n-p structure and $\mathrm{AlGaN} / \mathrm{SiC}$ heterojunction structure that the deep-UV response can be greatly enhanced. These APDs still exhibit high dark current at high bias voltage, which makes their performance in the high gain region inferior to SiC PIN APDs. The source of the dark current is believed to be related to high defect densities at the metal/SiC or $\mathrm{AlGaN} / \mathrm{SiC}$ interfaces. A direct chemical passivation on the $\mathrm{SiC}$ surface without introducing another materials in the high electric field region would be the best solution.

A very significant advantage of $\mathrm{SiC}$, over other wide bandgap semiconductors, is the high quality of the $\mathrm{SiO}_{2}$ layers grown by thermal oxidation. Usually, this thermally-grown $\mathrm{SiO}_{2}$ is used as the passivation material in the $\mathrm{SiC}$ APD fabrication. But the high surface recombination velocity indicates that there is still a high density of defects present at the $\mathrm{SiO}_{2} / \mathrm{SiC}$ interface, which result in a Fermi level pinning. Since $\mathrm{SiC}$ is widely used for high power metal-oxide-semiconductor field effect transistors, the researcher have done studies on the defects in the $\mathrm{SiO}_{2} / \mathrm{SiC}$ interface, which result in low channel mobility [7.1-7.3]. 
Studies have shown that there is large and broad density of states fixed at approximately $2.9 \mathrm{eV}$ above the valence band edge [7.4]. This high density of states in the forbidden band is attributed to a residue carbon complex that is released during thermal oxidation and remains at or near the $\mathrm{SiO}_{2} / \mathrm{SiC}$ interface. Different chemical treatments have been investigated to reduce the interface states density [7.5-7.6]. Among them, post-oxidation annealing in nitric oxide (NO) ambient is the most widely adopted method. It has been shown that nitric oxide annealing is effective in increasing the channel mobility and device reliability for SiC MOSFETs [7.7]. It is believed that it is the nitrogen atoms that passivate the interface by removing the carbon complex and creating strong $\mathrm{Si}-\mathrm{N}$ bonds [7.8].

It appears that both the low channel mobility in SiC MOSFETs and the high surface recombination velocity in $\mathrm{SiC}$ APDs are related to the high density of interface states between $\mathrm{SiO}_{2}$ and $\mathrm{SiC}$. It follows that a similar nitrogen passivation technique could be introduced in the SiC APD fabrication process. The tentative passivation process modification that I have designed is listed here:

1) RCA clean of the sample after mesa patterning

2) Load the sample into the oxidation furnace at a temperature of $1050 \mathrm{C}$ for an hour with a constant oxygen as flow

3) Change the gas from oxygen to nitric oxide and maintain the temperature for another hour

4) Cap the thermally grown oxide with $~ 300$ nm PECVD grown oxide 


\subsubsection{InGaP/AIGaAs UV-enhanced avalanche photodiode}

In chapter 5 I have demonstrated $\mathrm{Al}_{0.8} \mathrm{Ga}_{0.2} \mathrm{As}$ APDs with low dark current, high gain and low noise. The good linear-mode performance of these APDs confirmed that $\mathrm{Al}_{0.8} \mathrm{Ga}_{0.2} \mathrm{As}$ is a promising multiplication region material candidate to replace $\mathrm{Si}$ for the near-UV detection. However, the high surface recombination velocity of GaAs and AlGaAs prevented the photodiode from achieving high quantum efficiency [7.9]. Though sulfur passivation has proved to be effective in reducing the sidewall leakage, there is no evidence that this improves the quantum efficiency.

Inspired from the $\mathrm{AlGaN} / \mathrm{SiC}$ photodiode that is discussed in chapter 4, a heterojunction structure could be used to achieve better surface recombination velocity would utilize $\mathrm{In}_{0.49} \mathrm{Ga}_{0.51} \mathrm{P}$ which is lattice matched to GaAs/AlGaAs as the top surface layer. First, $\operatorname{In}_{0.49} \mathrm{Ga}_{0.51} \mathrm{P}$ has a reported surface recombination velocity of $\sim 10^{4} \mathrm{~cm} / \mathrm{s}$ [7.10], which is two orders of magnitude lower than GaAs. Second, the lattice match between $\mathrm{In}_{0.49} \mathrm{Ga}_{0.51} \mathrm{P}$ and $\mathrm{GaAs} / \mathrm{AlGaAs}$ will eliminate dislocation-related defects. An InGaP/GaAs photodiode utilizing a similar structure has been reported with quantum efficiency as high as $70 \%$ in the $360-870 \mathrm{~nm}$ range [7.11]. Despite the favorable electrical properties of $\operatorname{In}_{0.49} \mathrm{Ga}_{0.51} \mathrm{P}$, it also has one disadvantage for our purpose, which is a relatively high absorption coefficient $\left(\sim 5.5 \times 10^{5} \mathrm{~cm}^{-1}\right.$ at $\left.400 \mathrm{~nm}\right)$ [7.12]. In order to minimize the absorption in the $\operatorname{In}_{0.49} \mathrm{Ga}_{0.51} \mathrm{P}$ layer, a very thin layer is needed. The initial design of the structure is shown in Figure 7-1. A $50 \mathrm{~nm}$ GaAs contact layer is used for better ohmic contact formation, but the GaAs in the active area can be easily removed by the selective chemical etchant. 


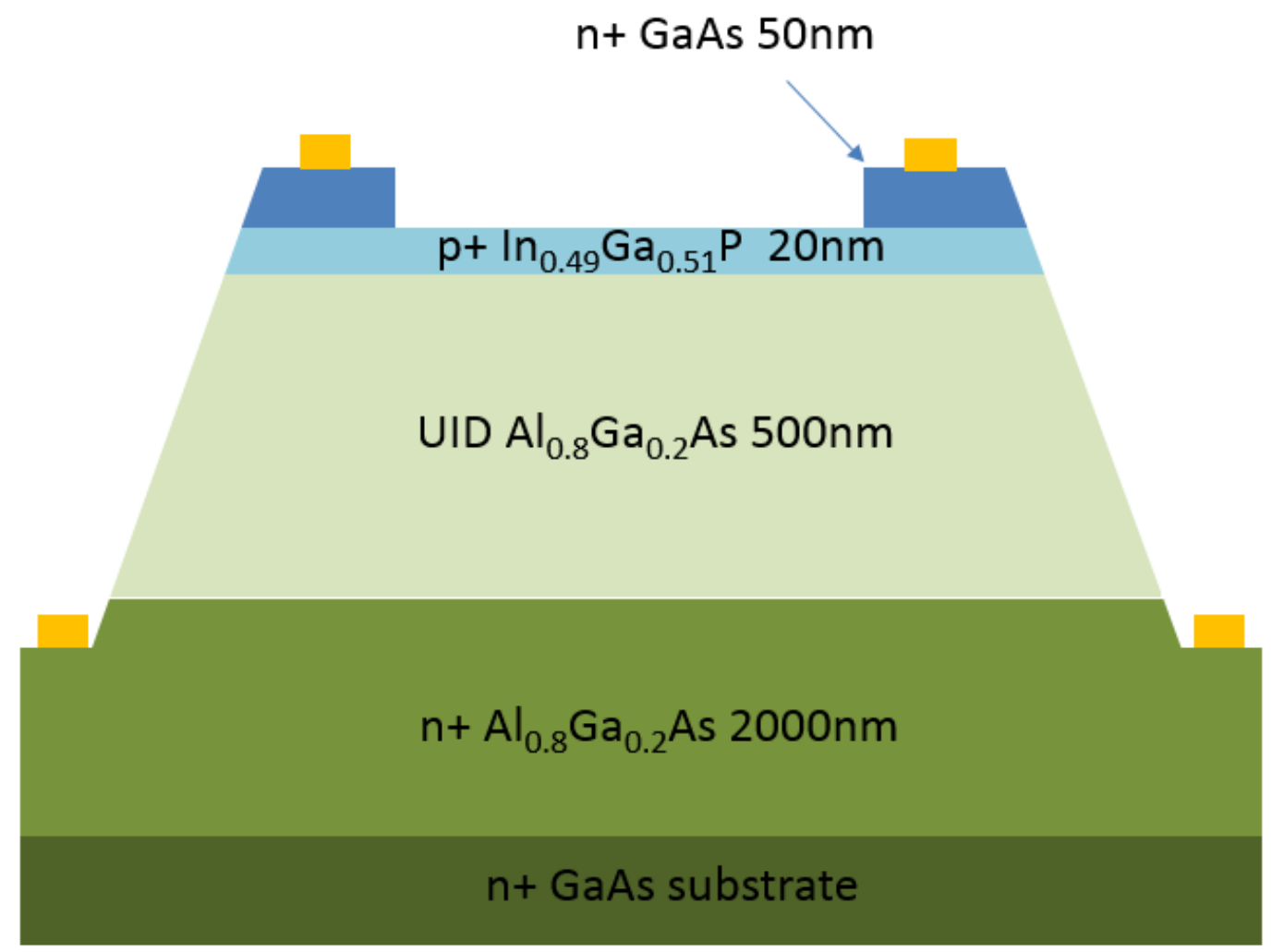

Figure 7-1. Cross section of the InGaP/AlGaAs APD 


\section{References}

[1.1] ISO 21348 Definitions of Solar Irradiance Spectral Categories

[1.2] C. A. Primmerman, "Detection of Biological Agents," Lincoln Lab. J., vol. 12, pp. 3-32, 2000

[1.3] G. A. Shaw, et al., "Recent progress in short-range ultraviolet communication," Proc. SPIE, vol. 5796, 28 March 2005.

[1.4] J. C. Campbell, et al., "Recent advances in avalanche photodiodes," Ieee Journal of Selected Topics in Quantum Electronics, vol. 10, pp. 777-787, Jul-Aug 2004.

[1.5] E.Monroy, et al.,"Wide-bandgap semiconductor ultraviolet photodetectos", Semicond. Sci. Technol. 18 (2003) R33-R51

[1.6] M. Razeghi, et al.,"Semiconductor ultraviolet detectors", J. Appl. Phys. 79 (10), 15 May 1996

[1.7] X. G. Bai, et al., "High detection sensitivity of ultraviolet 4H-SiC avalanche photodiodes," Ieee Journal of Quantum Electronics, vol. 43, pp. 1159-1162, NovDec 2007.

[1.8] Q. G. Zhou, et al., "Proton-Implantation-Isolated 4H-SiC Avalanche Photodiodes," Ieee Photonics Technology Letters, vol. 21, pp. 1734-1736, Dec 12009.

[1.9] X. Y. Guo, et al., "Demonstration of ultraviolet separate absorption and multiplication 4H-SiC avalanche photodiodes," Ieee Photonics Technology Letters, vol. 18, pp. 136-138, Jan-Feb 2006.

[1.10] H. D. Liu, et al., "4H-SiC PIN Recessed-Window Avalanche Photodiode With High Quantum Efficiency," Ieee Photonics Technology Letters, vol. 20, pp. 15511553, Sep-Oct 2008..

[2.1] R. J. McIntyre, "Multiplication noise in uniform avalanche diodes," Electron Devices, IEEE Transactions on 13, 5 (1966).

[3.1] Ng, B. K., et al. "Nonlocal effects in thin 4H-SiC UV avalanche photodiodes." Electron Devices, IEEE Transactions on 50.8 (2003): 1724-1732..

[3.2] Fitzgerald, D. J., and A. S. Grove. "Surface recombination in semiconductors." Electron Devices Meeting, 1967 International. Vol. 13. IEEE, 1967

[3.3] Galeckas, A., et al. "Optical characterization of excess carrier lifetime and surface recombination in 4H/6H-SiC." Applied Physics Letters 79.3 (2001): 365-367. 
[3.4] Cuevas, Andrés, et al. "Surface recombination velocity of highly doped n-type silicon." Journal of Applied Physics 80.6 (1996): 3370-3375.

[3.5] S.M.Sze. Physics of Semiconductor Devices. Third Edition

[3.6] Ohno, Takahisa. "Sulfur passivation of GaAs surfaces." Physical Review B44.12 (1991): 6306.

[3.7] Chang, K. J., and D. J. Chadi. "Theory of hydrogen passivation of shallow-level dopants in crystalline silicon." Physical review letters 60.14 (1988): 1422.

[3.8] Sciuto, Antonella, et al. "High responsivity 4H-SiC Schottky UV photodiodes based on the pinch-off surface effect." Applied physics letters 89.8 (2006): 081111081111.

[3.9] Xin, X., et al. "Demonstration of 4H-SiC visible-blind EUV and UV detector with large detection area." Electronics Letters 41.21 (2005): 1192-1193.

[4.1] Monroy, E., et al. "High-quality visible-blind AlGaN pin photodiodes." Applied physics letters 74.8 (1999): 1171-1173.

[4.2] Tut, Turgut, et al. "Experimental evaluation of impact ionization coefficients in AlxGa1- xN based avalanche photodiodes." Applied physics letters 89.18 (2006): 183524.

[4.3] Lin, C. F., et al. "Growth and characterizations of $\mathrm{GaN}$ on $\mathrm{SiC}$ substrates with buffer layers." Journal of applied physics 82.5 (1997): 2378-2382.

[4.4] Bernardini, Fabio, Vincenzo Fiorentini, and David Vanderbilt. "Spontaneous polarization and piezoelectric constants of III-V nitrides." Physical Review B 56.16 (1997): R10024.

[4.5] Rodak, L. E., et al. "AlGaN/SiC separate absorption and multiplication photodiode." Lester Eastman Conference on High Performance Devices (LEC), 2012. IEEE, 2012.

[4.6] Nepal, N., et al. "Temperature and compositional dependence of the energy band gap of AlGaN alloys." Applied Physics Letters 87.24 (2005): 242104-242104.

[4.7] Rodak, L. E., et al. "Solar-blind AlxGa1- xN/AlN/SiC photodiodes with a polarization-induced electron filter." Applied Physics Letters 103.7 (2013): 071110.

[5.1] Pepin, Catherine M., et al. "New UV-enhanced, ultra-low noise silicon avalanche photodiode for radiation detection and medical imaging." Nuclear Science Symposium Conference Record (NSS/MIC), 2010 IEEE. IEEE, 2010. 
[5.2] C McIntosh, Dion, et al. "High quantum efficiency $\mathrm{GaP}$ avalanche photodiodes." Optics express 19.20 (2011): 19607-19612.

[5.3] Anselm, K. A., et al. "Characteristics of GaAs and AlGaAs homojunction avalanche photodiodes with thin multiplication regions." Applied physics letters 71.26 (1997): 3883-3885.

[5.4] Robbins, V. M., S. C. Smith, and G. E. Stillman. "Impact ionization in AlxGa1xAs for $\mathrm{x}=0.1-0.4$." Applied physics letters 52.4 (1988): 296-298.

[5.5] Plimmer, Stephen A., et al. "Avalanche multiplication in Al x Ga 1-x As ( $\mathrm{x}=0$ to 0.60)." Electron Devices, IEEE Transactions on 47.5 (2000): 1089-1097.

[5.6] Lauter, J., et al. "AlGaAs/GaAs SAM-avalanche photodiode: an X-ray detector for low energy photons." Nuclear Instruments and Methods in Physics Research Section A: Accelerators, Spectrometers, Detectors and Associated Equipment 356.2 (1995): 324-329.

[5.7] Saleh, Mohammad A., et al. "Dead-space-based theory correctly predicts excess noise factor for thin GaAs and AlGaAs avalanche photodiodes."Electron Devices, IEEE Transactions on 47.3 (2000): 625-633.

[5.8] R B. K. Ng, J. P. R. David, S. A. Plimmer, G. J. Rees, R. C. Tozer, M. Hopkinson, and G. Hill, "Avalanche Multiplication Characteristics of $\mathrm{Al}_{0.8} \mathrm{Ga}_{0.2} \mathrm{As}$ Diodes," IEEE Trans. Electron Devices, vol. 48, 10, 2198 (2001).

[5.9] Oigawa, Haruhiro, et al. "Universal passivation effect of (NH4) 2Sx treatment on the surface of III-V compound semiconductors." Japanese journal of applied physics 30.3A (1991): L322.

[6.1] $\mathrm{Hu}, \mathrm{C}$, et al. "Noise characteristics of thin multiplication region GaAs avalanche photodiodes." Applied physics letters 69.24 (1996): 3734-3736.

[6.2] Inomata, Y., K. Fukui, and K. Shirasawa. "Surface texturing of large area multicrystalline silicon solar cells using reactive ion etching method." Solar Energy Materials and Solar Cells 48.1 (1997): 237-242.

[6.3] Zhao, Jianhua, et al. "19.8\% efficient "honeycomb" textured multicrystalline and 24.4\% monocrystalline silicon solar cells." Applied Physics Letters73.14 (1998): 1991-1993.

[6.4] Ke, Min-Yung, et al. "Application of nanosphere lithography to LED surface texturing and to the fabrication of nanorod LED arrays." Selected Topics in Quantum Electronics, IEEE Journal of 15.4 (2009): 1242-1249. 
[6.5] Windisch, R., et al. "Impact of texture-enhanced transmission on high-efficiency surface-textured light-emitting diodes." Applied physics letters79.15 (2001): 23152317.

[6.6] $\mathrm{Li}$, Zhi, et al. "Laser-textured silicon photodiode with broadband spectral response." Applied optics 50.17 (2011): 2508-2511.

[6.7] Zhou, Qiugui, et al. "Nanosphere natural lithography surface texturing as antireflective layer on $\mathrm{SiC}$ photodiodes." Optics express 19.24 (2011): 23664-23670.

[7.1] Chung, G. Y., et al. "Improved inversion channel mobility for 4H-SiC MOSFETs following high temperature anneals in nitric oxide." Electron Device Letters, IEEE 22.4 (2001): 176-178.

[7.2] Schörner, Reinhold, et al. "Enhanced channel mobility of 4H-SiC metal-oxidesemiconductor transistors fabricated with standard polycrystalline silicon technology and gate-oxide nitridation." Applied physics letters 80.22 (2002): 42534255 .

[7.3] Suzuki, Seiji, et al. "Correlation between channel mobility and shallow interface traps in SiC metal-oxide-semiconductor field-effect transistors." Journal of applied physics 92.10 (2002): 6230-6234.

[7.4] Chung, G. Y., et al. "Effect of nitric oxide annealing on the interface trap densities near the band edges in the $4 \mathrm{H}$ polytype of silicon carbide." Applied Physics Letters 76.13 (2000): 1713-1715.

[7.5] Senzaki, Junji, et al. "Excellent effects of hydrogen postoxidation annealing on inversion channel mobility of 4H-SiC MOSFET fabricated on $\left(\begin{array}{lll}1 & 2 & 0\end{array}\right)$ face." Electron Device Letters, IEEE 23.1 (2002): 13-15.

[7.6] Okamoto, Dai, et al. "Improved inversion channel mobility in 4H-SiC MOSFETs on Si face utilizing phosphorus-doped gate oxide." Electron Device Letters, IEEE 31.7 (2010): 710-712.

[7.7] Rozen, John, et al. "Scaling between channel mobility and interface state density in SiC MOSFETs." Electron Devices, IEEE Transactions on 58.11 (2011): 38083811.

[7.8] Jamet, Philippe, Sima Dimitrijev, and Philip Tanner. "Effects of nitridation in gate oxides grown on 4H-SiC." Journal of Applied Physics 90.10 (2001): 5058-5063.

[7.9] Nelson, R. J., et al. "Reduction of GaAs surface recombination velocity by chemical treatment." Applied Physics Letters 36.1 (1980): 76-79. 
[7.10] Pearton, S. J., et al. "Surface recombination velocities on processed InGaP p-n junctions." Applied physics letters 63.26 (1993): 3610-3612.

[7.11] Wu, Meng-Chyi, Yun-Hsun Huang, and Chong-Long Ho. "High-speed InGaP/GaAs pin Photodiodes with wide spectral range." Electron Device Letters, IEEE 28.9 (2007): 797-799.

[7.12] Kato, Hirokazu, et al. "Optical properties of (AlxGa1-x) 0.5 In0. 5P quaternary alloys." Japanese journal of applied physics 33.1R (1994): 186. 


\section{Appendix. Quantum Efficiency Modeling Code}

Total_QE.m: Set all the parameters and device structure, calculate the total QE

Top_Layer_QE.m: Calculate the QE due to absorption in the undepleted top layer

Depletion_Layer_QE.m: Calculate the QE due to absorption in the depleted layer

Bottom_Layer_QE.m: Calculate the QE due to absorption in the undepleted bottom

layer

Get_AbsorptionCoefficient.m: Get absorption coefficient for each wavelength

Get_Reflectance.m: Get reflectance for each wavelength.

\section{Total_QE.m}

$\% \%$ Set each layers' thickness

$\mathrm{X} 1$ = 260e-7;

$\mathrm{X} 2=170 \mathrm{e}-7+\mathrm{X} 1$;

$\mathrm{X} 3=500 \mathrm{e}-7+\mathrm{X} 2$;

$\% \%$ Set the diffusion length and diffusion constant for electrons and holes, here are the

numbers for $\mathrm{Al}_{0.8} \mathrm{Ga}_{0.2} \mathrm{As}$

Diff_Length_H = 200e-7;

Diff_Length_E $=80 \mathrm{e}-7$;

Diff_Const_H $=1.84$;

Diff_Const_E $=5.28$;

$\% \%$ Set surface recombination velocity

Surf_Recom_V=9e8; 
$\% \%$ Set start and stop wavelength

Wavelength_start $=300$;

Wavelength_end $=550$;

$\mathrm{N}=($ Wavelength_end-Wavelength_start)/10+1;

$\% \%$ Create space for $\mathrm{QE}$

$\mathrm{QE} 1=[1: \mathrm{N}]$;

$\mathrm{QE} 2=[1: \mathrm{N}]$

$\mathrm{QE} 3=[1: \mathrm{N}]$;

$\% \%$ Create space for wavelength, reflectance and absorption coefficient

Wavelength=Wavelength_start:10:Wavelength_end;

Reflect $=$ ones $(1,($ Wavelength_end-Wavelength_start $) / 10+1)$;

Absorp_Coeff=ones(1,(Wavelength_end-Wavelength_start)/10+1);

for $\mathrm{m} \_\mathrm{W}=1$ :(Wavelength_end-Wavelength_start)/10+1

$\operatorname{Absorp} \_C o e f f\left(m \_W\right)=$ get_absorptioncoefficient $\left(\right.$ Wavelength $\left.\left(\mathrm{m} \_\mathrm{W}\right)\right)$;

$\operatorname{Reflect}\left(\mathrm{m} \_\mathrm{W}\right)=$ get_reflectance $\left(\right.$ Wavelength $\left.\left(\mathrm{m} \_\mathrm{W}\right)\right)$;

end

$\% \%$ Calculate QE due to absorption from different layers

for $\mathrm{n}=1$ :(Wavelength_end-Wavelength_start)/10+1

\%\% Top_Layer_QE(Absorp_Coeff, X1, Diff_Length,Diff_Const, Surf_Recom_V)

QE1(n) = Top_Layer_QE(Absorp_Coeff(n), X1, Diff_Length_E, Diff_Const_E, Surf_Recom_V);

\%\% Depletion_Layer_QE(Absorp_Coeff, X1, X2) Absorption Layer

$\mathrm{QE} 2(\mathrm{n})=$ Depletion_Layer_QE( Absorp_Coeff(n), X1, X2); 
$\% \%$ Bottom_Layer_QE(Absorp_Coeff, X2, X3, Diff_Length, Diff_Const, Surf_Recom_V)

$$
\text { QE3(n) = Bottom_Layer_QE(Absorp_Coeff(n), X2, X3, Diff_Length_H, }
$$
Diff_Const_H, 0);

end

$\% \%$ Plot the graph

simulatedQE $=(\mathrm{QE} 1+\mathrm{QE} 2+\mathrm{QE} 3)$.*(1-Reflect $) * 100$;

plot(Wavelength,simulatedQE, 'black');

\section{Top_Layer_QE.m}

$\% \%$ Calculate QE from top layer thickness, absorption coefficient, diffusion length,

$\% \%$ diffusion constant and surface recombination velocity

function QE = Top_Layer_QE(Absorp_Coeff, X, Diff_Length, Diff_Const, Surf_Recom_V)

aa = Absorp_Coeff*Diff_Length/(Absorp_Coeff^ $2 *$ Diff_Length^2-1);

$\mathrm{bb}=($ Absorp_Coeff*Diff_Const + Surf_Recom_V $)-\exp (-$ Absorp_Coeff*X)*(

Surf_Recom_V* $\cosh \left(\mathrm{X} / \mathrm{Diff} \_L e n g t h\right)+$ Diff_Const/Diff_Length*sinh(X/Diff_Length)

)

$\mathrm{cc}=\left(\right.$ Surf_Recom_V* $\sinh \left(\mathrm{X} / \mathrm{Diff} \_\right.$Length $)+$

Diff_Const/Diff_Length*cosh(X/Diff_Length) );

dd = Absorp_Coeff*Diff_Length*exp(-Absorp_Coeff*X); 
$\mathrm{QE}=\mathrm{aa} *(\mathrm{bb} / \mathrm{cc}-\mathrm{dd})$

\section{Depletion_Layer_QE.m}

$\% \%$ Calculate the $\mathrm{QE}$ from the depleted layer, assuming all the photo generated carriers $\% \%$ in this layer contribute to the $\mathrm{QE}$

function $\mathrm{QE}=$ Depletion_Layer_QE(Absorp_Coeff, X1, X2)

$\mathrm{QE}=\exp ($-Absorp_Coeff * X1)*(1- exp (-Absorp_Coeff*(X2 - X1) ) );

\section{Bottom_Layer_QE.m}

$\% \%$ Calculate the $\mathrm{QE}$ due to absorption in the bottom layer using the all three layers' $\% \%$ thickness, absorption coefficient, diffusion length and surface recombination $\% \%$ velocity function QE = Bottom_Layer_QE(Absorp_Coeff, X2, X3, Diff_Length, Diff_Const, Surf_Recom_V)

Kn_aa $=$ Surf_Recom_V $*\left(\exp \left(-\operatorname{Absorp} \_C o e f f *(X 3-X 2)\right)-\cosh ((X 3-\right.$ X2)/Diff_Length ) );

Kn_bb $=$ Absorp_Coeff $*$ Diff_Const $* \exp (-$ Absorp_Coeff $*(X 3-X 2))$;

Kn_cc $=$ Diff_Const $/$ Diff_Length $* \sinh ((X 3-X 2) /$ Diff_Length $)$;

Kn_dd $=$ Diff_Const $/$ Diff_Length $* \cosh ((X 3-X 2) /$ Diff_Length $)$;

Kn_ee $=$ Surf_Recom_V $* \sinh ((X 3-X 2) /$ Diff_Length $)$; 
$\mathrm{Kn}=\left(\mathrm{Kn} \_\mathrm{aa}-\mathrm{Kn} \_\mathrm{bb}-\mathrm{Kn} \_c c\right) /\left(\mathrm{Kn} \_\mathrm{dd}+\mathrm{Kn} \_\right.$ee $)$;

QE_aa $=$ Absorp_Coeff $*$ Diff_Length;

$\mathrm{QE}=\mathrm{QE} \_\mathrm{aa} * \exp \left(-\operatorname{Absorp} \_C o e f f * X 2\right) /\left(\mathrm{QE} \_\mathrm{aa}{ }^{\wedge} 2-1\right) *\left(\mathrm{QE} \_\mathrm{aa}+\mathrm{Kn}\right)$;

\section{Get_AbsorptionCoefficient.m}

$\% \%$ Get the absorption coefficient at given wavelength, data is from published literature function absorption=get_absorptioncoefficient(Wavelength)

\begin{tabular}{|c|c|c|c|c|c|c|c|c|c|c|}
\hline Lamda $=[300$ & 310 & 320 & 330 & 340 & 350 & 360 & 370 & 380 & 390 & 400 \\
\hline 410 & 420 & 430 & 440 & 450 & 460 & 470 & 480 & 490 & 500 & 510 \\
\hline 520 & 530 & 540 & $550]$ & & & & & & & \\
\hline
\end{tabular}

$\mathrm{Alfa}=[8.95 \mathrm{e} 5$ 8.37e5 8.00e5 7.16e5 5.99e5 4.69e5 3.51e5 2.63e5 2.06e5 1.65e5 1.35e5

$1.13 \mathrm{e} 59.44 \mathrm{e} 4$ 7.55e4 5.91e4 5.17e4 4.20e4 3.24e4 $1.94 \mathrm{e} 46.67 \mathrm{e} 32.5 \mathrm{e} 3 \quad 1.37 \mathrm{e} 3 \quad 8.22 \mathrm{e} 2$

$7.19 \mathrm{e} 26.29 \mathrm{e} 25.75 \mathrm{e} 2]$

absorption=exp(interp1(Lamda, $\log ($ Alfa $)$, Wavelength,'linear',1));

\section{Get_Reflectance.m}

$\% \%$ Get reflectance spectrum, data is from reflectance measurement in the lab

function R=get_reflectance(Wavelength)

\begin{tabular}{|c|c|c|c|c|c|c|c|c|c|}
\hline Lamda $=[300$ & 310 & 320 & 330 & 340 & 350 & 360 & 370 & 380 & 390 \\
\hline 410 & 420 & 430 & 440 & 450 & 460 & 470 & 480 & 490 & 500 \\
\hline 520 & 530 & 540 & 550]; & & & & & & \\
\hline
\end{tabular}




$\begin{aligned} & \text { Reflectance= }\left.\begin{array}{rrrrrrrrrrr}45.5 & 45.6 & 46.0 & 46.3 & 45.9 & 45.0 & 43.3 & 41.6 & 40.1 & 38.9 \\ 37.9 & 37.0 & 36.3 & 35.7 & 35.0 & 34.4 & 33.9 & 33.6 & 33.3 & 32.8 & 32.0 \\ 31.4 & 30.9 & 30.5 & 30.2 & 29.9\end{array}\right] ; \\ & \text { R=interp1(Lamda,Reflectance,Wavelength,'linear',1)/100; }\end{aligned}$

